\title{
Removal of violations of the Master Ward Identity in perturbative QFT
}

\author{
Ferdinand Brennecke \\ Institut für Quantenelektronik, ETH Zürich \\ CH-8093 Zürich, Switzerland \\ brennecke@phys.ethz.ch \\ Michael Dütsch \\ Institut für Theoretische Physik, Universität Zürich \\ CH-8057 Zürich, Switzerland \\ and Max Planck Institute for Mathematics in the Sciences \\ D-04103 Leipzig, Germany \\ duetsch@physik.unizh.ch
}

\begin{abstract}
We study the appearance of anomalies of the Master Ward Identity, which is a universal renormalization condition in perturbative QFT. The main insight of the present paper is that any violation of the Master Ward Identity can be expressed as a local interacting field; this is a version of the well-known Quantum Action Principle of Lowenstein and Lam. Proceeding in a proper field formalism by induction on the order in $\hbar$, this knowledge about the structure of possible anomalies as well as techniques of algebraic renormalization are used to remove possible anomalies by finite renormalizations. As an example the method is applied to prove the Ward identities of the $O(N)$ scalar field model.

PACS. 11.10.Cd Field theory: Axiomatic approach, 11.10.Gh Field theory: Renormalization, 11.15.Bt Gauge field theories: General properties of perturbation theory, 11.30.-j Field theory: Symmetry and conservation laws
\end{abstract}

\section{Contents}

1 Introduction $\quad 2$

2 Classical field theory for localized interactions $\quad 4$

3 Perturbative Quantum Field Theory $\quad 9$

4 Proper vertices for $T$-products $\quad 14$ 
4.1 Diagrammatics and definition of the 1-particle-irreducible part $T^{1 \mathrm{PI}}$ of

the time ordered product . . . . . . . . . . . . . . . 14

4.2 Definition and basic properties of the vertex function $\Gamma_{T} \ldots \ldots$. . . . 17

4.3 Comparison with the literature . . . . . . . . . . . . 20

5 The Master Ward Identity 21

5.1 The classical MWI in the off-shell formalism . . . . . . . . . . . . . . 21

5.2 Structure of possible anomalies of the MWI in QFT . . . . . . . . . . 23

5.3 The MWI in the proper field formalism, and the Quantum Action Principle 32

5.4 Removal of violations of the MWI . . . . . . . . . . . . . . . 34

5.4.1 Fulfillment of the MWI to first order in $S$. . . . . . . . . . 34

5.4.2 Removal of possible anomalies by induction on the order in $\hbar$. . 35

5.4.3 Assumption: localized off-shell version of Noether's Theorem . . 36

5.4.4 Proof of the Ward identities in the $O(N)$ scalar field model . . . 40

6 Conclusions and Outlook

A Proper vertices for $R$-products $\quad 43$

A.1 Definition and basic properties . . . . . . . . . . . . . 43

A.2 Comparison of the vertex functions in terms of $T$ - and $R$-products . . . 47

\section{Introduction}

In the quantization of a classical field theory symmetries and corresponding conservation laws are in general not maintained: due to the distributional character of quantum fields the arguments valid for classical field theory are not applicable. Therefore, in perturbative quantum field theory (pQFT) symmetries and conservation laws play the role of renormalization conditions (the 'Ward identities').

In [1] a universal formulation of Ward identities was studied and termed Master Ward Identity (MWI). This identity - which originally was proposed in [2] - can be derived in the framework of classical field theory simply from the fact that classical fields can be multiplied pointwise. However, in pQFT the MWI serves as a highly non-trivial renormalization condition, which cannot be fulfilled in general due to the well known anomalies appearing in QFT.

In traditional renormalization theory (e.g. BPHZ renormalization or dimensional renormalization) the question whether certain Ward identities can be fulfilled, is usually treated by means of algebraic renormalization ${ }^{1}$. This method relies on the Quantum Action Principle (QAP), which was derived by Lowenstein and Lam in the early seventies [3, 4] and proved in several renormalization schemes [5]. The QAP characterizes the possible violations of Ward identities and, hence, allows one to derive algebraic conditions whose solvability guarantees the existence of a renormalization maintaining the Ward identities. These conditions often lead to cohomological problems involving the infinitesimal symmetry operators which appear in the considered Ward identities.

\footnotetext{
${ }^{1}$ There is a huge literature about algebraic renormalization. For shortness we cite only some of the founding articles and a few textbooks and reviews, which should suffice to understand this paper.
} 
The application of this procedure to Yang-Mills theories lead to a detailed study of BRST cohomology $[6,7,8,9,10,11]$.

Traditionally, perturbation theory is done in the functional formulation of QFT which starts from the path integral. However, we take the point of view of algebraic pQFT $[12,13,14,15,16]$, which is based on causal perturbation theory (Bogoliubov [17], Epstein and Glaser [18]) and concentrates on the algebraic structure of interacting fields. Starting with some well defined free QFT one separates UV-problems from IR-problems by considering solely interactions with compact support. Whereas the UV-problem concerns the construction of time ordered or retarded products (in terms of which interacting fields are formulated), the IR-problem appears only in the construction of states on the algebra of local observables. The restriction on compactly supported interactions leaves it possible to construct the whole net of local observables [12]. Therefore, this approach seems to be well suited for a rigorous perturbative construction of quantum Yang-Mills theories, for example, where an adiabatic limit seems to be out of reach. However, in the non-Abelian case the construction of the net of local observables is still an open problem within the framework of algebraic pQFT. As it was worked out in [2], the decisive input to reach this goal is the MWI resp. certain cases of it. Motivated by this, the aim of our present work is to transfer techniques from algebraic renormalization theory into the framework of algebraic pQFT in order to gain more insight into the violations of the MWI and to find concrete conditions for the solvability of the MWI in relevant cases.

The paper is organized as follows: Sect. 2 deals with classical field theory for localized interactions. We generalize the treatment given in Sect. 2 of [1] to the off-shell formalism, i.e. the values of the retarded products are off-shell fields. In Sect. 3 we summarize the quantization of perturbative classical field theory worked out in [16] and give some completions. Algebraic renormalization proceeds in terms of the 'vertex functional' (or 'proper function') $\Gamma$ (which is usually derived in the functional formulation of QFT along a Legendre transformation). Hence, to make accessible techniques of algebraic renormalization, we develop a proper field formalism, which describes the combinatorics of 1-particle-irreducible (1PI) diagrams in a purely algebraic setting (Sect. 4). This is done by reformulating perturbative QFT as a classical field theory with a non-local interaction $\Gamma$ which is a formal power series in $\hbar$.

After these preparatory sections we turn in Sect. 5 to the maintenance of the MWI in the process of renormalization. Starting from a derivation of the MWI in the off-shell formalism [16], we prove an identity ('anomalous MWI') which gives a characterization of the possible violations of the MWI. More precisely, we find that the most general violation can be expressed in terms of a local interacting field. Translation of the anomalous MWI into the proper field formalism (introduced in Sect. 4) yields an identity which contains solely the 'quantum part' (loop part) of the original version and which is shown to be formally equivalent to the QAP. Crucial properties of the violating local terms appearing in the anomalous MWI are proved, in particular an upper bound for the mass dimension. Equipped with this new insight, we transfer basic ideas of algebraic renormalization into the setting of causal perturbation theory. Usually, in the latter the maintenance of Ward identities in renormalization is proved by induction on the power of the coupling constant. In contrast, we proceed by induction on the power of $\hbar$ similarly to algebraic renormalization. We find explicit 
conditions, whose solvability guarantees the existence of a renormalization prescription satisfying corresponding cases of the MWI. In addition, we prove that the MWI can always be fulfilled to first order in the coupling constant (that is to second order of the corresponding time ordered products). We apply these results to models fulfilling (classically) a localized off-shell version of Noether's Theorem and find simplifications of the mentioned conditions. Finally, as a simple application of the method, we prove the Ward identities of the $O(N)$ scalar field model by using cohomological arguments.

\section{Classical field theory for localized interactions}

In this section we generalize the formalism developed in Sect. 2 of [1] to off-shell fields. This will provide us with the necessary framework to derive the off-shell version of the MWI in Sect. 5.1. In order to keep the formulas as simple as possible, we study the theory of a real scalar field $\varphi$ on $d$ dimensional Minkowski space $\mathbb{M}, d>2$. We interpret $\varphi$ and partial derivatives thereof as evaluation functionals on the configuration space $\mathcal{C} \equiv \mathcal{C}^{\infty}(\mathbb{M}, \mathbb{R}):\left(\partial^{a} \varphi\right)(x)(h)=\partial^{a} h(x), a \in \mathbb{N}_{0}^{d}$. Let $\mathcal{F}(\mathcal{C})$ be the space of all functionals

$$
F(\varphi): \mathcal{C} \longrightarrow \mathbb{C}, \quad F(\varphi)(h)=F(h)
$$

which are localized polynomials ${ }^{2}$ in $\varphi$ :

$$
F(\varphi)=\sum_{n=0}^{N} \int d x_{1} \ldots d x_{n} \varphi\left(x_{1}\right) \cdots \varphi\left(x_{n}\right) f_{n}\left(x_{1}, \ldots, x_{n}\right)=: \sum_{n}\left\langle f_{n}, \varphi^{\otimes n}\right\rangle,
$$

where $N<\infty$ and the $f_{n}$ 's are $\mathbb{C}$-valued distributions with compact support, which are symmetric under permutations of the arguments and whose wave front sets satisfy the condition ${ }^{3}$

$$
\mathrm{WF}\left(f_{n}\right) \cap\left(\mathbb{M}^{n} \times\left(\bar{V}_{+}^{n} \cup \bar{V}_{-}^{n}\right)\right)=\emptyset
$$

and $f_{0} \in \mathbb{C} . \mathcal{F}$ is a commutative algebra with the classical product $\left(F_{1} \cdot F_{2}\right)(h):=$ $F_{1}(h) \cdot F_{2}(h)$. By the support of a functional $F \in \mathcal{F}$ we mean the support of $\frac{\delta F}{\delta \varphi}$.

The space of local functionals $\mathcal{F}_{\text {loc }}(\mathcal{C}) \subset \mathcal{F}(\mathcal{C})$ is defined as

$$
\mathcal{F}_{\text {loc }}(\mathcal{C}) \stackrel{\text { def }}{=}\left\{\int d x \sum_{i=1}^{N} A_{i}(x) h_{i}(x) \equiv \sum_{i=1}^{N} A_{i}\left(h_{i}\right) \mid A_{i} \in \mathcal{P}, h_{i} \in \mathcal{D}(\mathbb{M})\right\}
$$

where $\mathcal{P}$ is the space of all polynomials of the field $\varphi$ and its partial derivatives. This representation of local functionals as smeared fields can be made unique by introducing the subspace of balanced fields $\mathcal{P}_{\text {bal }} \subset \mathcal{P}[16,20]$ :

$$
\mathcal{P}_{\text {bal }} \stackrel{\text { def }}{=}\left\{\left.P\left(\partial_{1}, \ldots, \partial_{n}\right) \varphi\left(x_{1}\right) \cdots \varphi\left(x_{n}\right)\right|_{x_{1}=\ldots=x_{n}=x} \mid P\left(\partial_{1}, \ldots, \partial_{n}\right) \in P_{n}^{\text {rel }}, n \in \mathbb{N}_{0}\right\}
$$

\footnotetext{
${ }^{2} \mathrm{~A}$ generalization to non-polynomial (localized) interactions is possible, see e.g. [19].

$3 \bar{V}_{ \pm}$denotes the closure of the forward and backward light-cones, respectively, and $\bar{V}_{ \pm}^{n}$ their $n$-fold direct products.
} 
where $P_{n}^{\text {rel }}$ is the space of all polynomials in the 'relative derivatives' $\left(\partial_{k}-\partial_{l}\right), 1 \leq$ $k<l \leq n$. With that it holds: given $F \in \mathcal{F}_{\text {loc }}$ there exists a unique $h \in \mathcal{D}\left(\mathbb{M}, \mathcal{P}_{\text {bal }}\right)$ with $\left.h\right|_{\varphi=0}=0$ and $F-F(0)=\int d x h(x)$. Proofs are given in Proposition 3.1 of [16] and in Lemma 1 of [20].

Since we are mainly interested in perturbation theory we consider action functionals of the form $S_{\text {tot }}=S_{0}+\lambda S$ where $S_{0} \stackrel{\text { def }}{=} \int d x \frac{1}{2}\left(\partial_{\mu} \varphi \partial^{\mu} \varphi-m^{2} \varphi^{2}\right)$ denotes the free action, $\lambda$ a real parameter and $S \in \mathcal{F}(\mathcal{C})$ is some compactly supported interaction, which may be non-local. ${ }^{4}$ We denote by $\Delta_{S_{\text {tot }}}^{\text {ret }}$ the retarded Green function corresponding to the action $S_{\text {tot }}$, which is defined by

$$
\int d y \Delta_{S_{\mathrm{tot}}}^{\mathrm{ret}}(x, y) \frac{\delta^{2} S_{\mathrm{tot}}}{\delta \varphi(y) \delta \varphi(z)}=\delta(x-z)=\int d y \frac{\delta^{2} S_{\mathrm{tot}}}{\delta \varphi(x) \delta \varphi(y)} \Delta_{S_{\mathrm{tot}}}^{\mathrm{ret}}(y, z)
$$

and $\Delta_{S_{\text {tot }}}^{\text {ret }}(x, y)=0$ for $x$ sufficiently early. In the following we will assume that for all actions $S_{\text {tot }}$ under consideration the retarded Green function exists and is unique in the sense of formal power series in $\lambda$. Analytic expressions for $\Delta_{S_{\text {tot }}}^{\text {ret }}$ are in general unknown. However, perturbatively the retarded solution of (2.6) can be given in terms of the (unique) retarded Green function $\Delta_{S_{0}}^{\mathrm{ret}}(x, y)=\Delta_{m}^{\mathrm{ret}}(x-y)$ of the Klein Gordon operator:

Lemma 1. In the sense of formal power series in $\lambda$, the retarded Green function $\Delta_{S_{0}+\lambda S}^{\mathrm{ret}}$ is given by the following formula [1]:

$$
\begin{gathered}
\Delta_{S_{0}+\lambda S}^{\mathrm{ret}}(x, y)=\Delta_{S_{0}}^{\mathrm{ret}}(x, y)+\sum_{n=1}^{\infty}(-\lambda)^{n} \int d\left(u_{1}, \ldots, u_{n}\right) d\left(v_{1}, \ldots, v_{n}\right) \Delta_{S_{0}}^{\mathrm{ret}}\left(x, u_{1}\right) \\
\frac{\delta^{2} S}{\delta \varphi\left(u_{1}\right) \delta \varphi\left(v_{1}\right)} \cdots \Delta_{S_{0}}^{\mathrm{ret}}\left(v_{n-1}, u_{n}\right) \frac{\delta^{2} S}{\delta \varphi\left(u_{n}\right) \delta \varphi\left(v_{n}\right)} \Delta_{S_{0}}^{\mathrm{ret}}\left(v_{n}, y\right)
\end{gathered}
$$

Its support is contained in the set

$$
\left\{(x, y) \mid x \in \operatorname{supp}\left(\frac{\delta S}{\delta \varphi}\right)+\bar{V}_{+} \wedge y \in \operatorname{supp}\left(\frac{\delta S}{\delta \varphi}\right)+\bar{V}_{-}\right\} \cup\left\{(x, y) \mid x \in y+\bar{V}_{+}\right\} .
$$

In case of a local interaction $S \in \mathcal{F}_{\text {loc }}(\mathcal{C})$ the support can be limited even stronger:

$$
\operatorname{supp}\left(\frac{\delta \Delta_{S_{0}+S}^{\mathrm{ret}}}{\delta \varphi}\right) \subset\left\{(x, y) \mid x \in y+\bar{V}_{+}\right\}
$$

Our assumption ensures that the pointwise product of distributions in (2.7) exists.

Proof. We multiply the left equation in $(2.6)$ by $\Delta_{S_{0}}^{\text {ret }}(z, u)$ and integrate over $z$ afterwards to obtain the relation

$$
\Delta_{S_{0}+\lambda S}^{\mathrm{ret}}(x, u)=\Delta_{S_{0}}^{\mathrm{ret}}(x, u)-\lambda \int d y d z \Delta_{S_{0}+\lambda S}^{\mathrm{ret}}(x, y) \frac{\delta^{2} S}{\delta \varphi(y) \delta \varphi(z)} \Delta_{S_{0}}^{\mathrm{ret}}(z, u)
$$

\footnotetext{
${ }^{4}$ Note that the free action is not an element of $\mathcal{F}(\mathcal{C})$. Therefore we interpret $S_{0}$ as functional on the subspace of compactly supported functions in configuration space $\mathcal{C}$. However, this restriction is not necessary (and, hence, will not be done) for the free field equation: $\frac{\delta S_{0}}{\delta \varphi}$ is an element of $\mathcal{P}$.
} 
which can be solved by recursion on the powers of $\lambda$ (2.7). For local interactions $S \in$ $\mathcal{F}_{\text {loc }}(\mathcal{C})$, the support property $(2.9)$ follows immediately from $\operatorname{supp}\left(\frac{\delta \Delta_{S_{0}}^{\text {ret }}}{\delta \varphi}\right) \subset\{(x, y) \mid x \in$ $\left.y+\bar{V}_{+}\right\}$and $\frac{\delta^{2} S}{\delta \varphi(u) \delta \varphi(v)}=0$ for $u \neq v$. In the general case $S \in \mathcal{F}(\mathcal{C})$ we use $\operatorname{supp}\left(\frac{\delta^{2} S}{\delta \varphi^{2}}\right) \subset$ $\operatorname{supp}\left(\frac{\delta S}{\delta \varphi}\right) \times \operatorname{supp}\left(\frac{\delta S}{\delta \varphi}\right)$ to conclude that $x$ has to lie in the future of $u_{1} \in \operatorname{supp}\left(\frac{\delta S}{\delta \varphi}\right)$ and $y$ in the past of $v_{n} \in \operatorname{supp}\left(\frac{\delta S}{\delta \varphi}\right)$ to get a non vanishing contribution of the integral in (2.7).

The space of all smooth solutions of the Euler-Lagrange equation with respect to the action $S_{\text {tot }}$ will be denoted by $\mathcal{C}_{S_{\text {tot }}} \subset \mathcal{C}$. Interacting fields $F_{S}$, corresponding to some functional $F \in \mathcal{F}(\mathcal{C})$, could be defined by restricting $F$ to the space of solutions $\mathcal{C}_{S_{0}+S}:\left.F_{S} \stackrel{\text { def }}{=} F\right|_{\mathcal{C}_{S_{0}+S}}$. However, the idea of perturbative algebraic classical field theory is to introduce interacting fields as functionals on the space $\mathcal{C}_{S_{0}}$ of free solutions. This corresponds to the usual interaction picture known from QFT where interacting fields are constructed as operators on the Fock space of the underlying free theory. Therefore one introduces retarded wave operators which map solutions of the free theory to solutions of the interacting theory [1]. As it turns out, it is convenient to construct such a map on the space $\mathcal{C}$ of all field configurations (off-shell formalism) and not only on the space of solutions, as it was done in [1]:

Definition 1. A retarded wave operator is a family of maps $\left(r_{S_{0}+S, S_{0}}\right)_{S \in \mathcal{F}(\mathcal{C})}$ from $\mathcal{C}$ into itself with the properties

(i) $r_{S_{0}+S, S_{0}}(f)(x)=f(x)$ for $x$ sufficiently early

(ii) $\frac{\delta\left(S_{0}+S\right)}{\delta \varphi} \circ r_{S_{0}+S, S_{0}}=\frac{\delta S_{0}}{\delta \varphi}$.

Lemma 2. The retarded wave operator $\left(r_{S_{0}+S, S_{0}}\right)_{S \in \mathcal{F}(\mathcal{C})}$ exists and is unique and invertible in the sense of formal power series in the interaction $S$.

Proof. To determine $r_{S_{0}+S, S_{0}}$ we multiply $S$ in Def. 1, (ii) by a real parameter $\lambda$ and differentiate with respect to this parameter, ending up with

$$
\int d y \frac{\delta\left(S_{0}+\lambda S\right)}{\delta \varphi(x) \delta \varphi(y)} \circ r_{S_{0}+\lambda S, S_{0}} \cdot \varphi(y) \circ \frac{d}{d \lambda} r_{S_{0}+\lambda S, S_{0}}+\frac{\delta S}{\delta \varphi(x)} \circ r_{S_{0}+\lambda S, S_{0}}=0 .
$$

After multiplication of this equation with $\Delta_{S_{0}+\lambda S}^{\text {ret }}(z, x)$ and integration over $x$, we obtain the following differential equation for $r_{S_{0}+\lambda S, S_{0}}$

$$
\varphi(z) \circ \frac{d}{d \lambda} r_{S_{0}+\lambda S, S_{0}}=-\int d x\left(\Delta_{S_{0}+\lambda S}^{\mathrm{ret}}(z, x) \cdot \frac{\delta S}{\delta \varphi(x)}\right) \circ r_{S_{0}+\lambda S, S_{0}} .
$$

Finally, integration over $\lambda$ leads to the equation

$$
\varphi(z) \circ r_{S_{0}+S, S_{0}}=\varphi(z)-\int_{0}^{1} d \lambda \int d x\left(\Delta_{S_{0}+\lambda S}^{\mathrm{ret}}(z, x) \cdot \frac{\delta S}{\delta \varphi(x)}\right) \circ r_{S_{0}+\lambda S, S_{0}},
$$

which can be solved iteratively in the sense of formal power series in the interaction $S$. 
We define the retarded wave operator $r_{S_{0}+S_{1}, S_{0}+S_{2}}$ connecting two interacting theories by

$$
r_{S_{0}+S_{1}, S_{0}+S_{2}}:=r_{S_{0}+S_{1}, S_{0}} \circ\left(r_{S_{0}+S_{2}, S_{0}}\right)^{-1} \text {. }
$$

Obviously it fulfills Def. $1(i)$ and $\frac{\delta\left(S_{0}+S_{1}\right)}{\delta \varphi} \circ r_{S_{0}+S_{1}, S_{0}+S_{2}}=\frac{\delta\left(S_{0}+S_{2}\right)}{\delta \varphi}$ and

$$
r_{S_{0}+S_{1}, S_{0}+S_{2}} \circ r_{S_{0}+S_{2}, S_{0}+S_{3}}=r_{S_{0}+S_{1}, S_{0}+S_{3}} .
$$

Retarded fields (to the interaction $S$ and the free theory $S_{0}$ and corresponding to the functional $F \in \mathcal{F}(\mathcal{C}))$ are defined by

$$
F_{S_{0}, S}^{\text {ret }} \stackrel{\text { def }}{=} F \circ r_{S_{0}+S, S_{0}}: \mathcal{C} \longrightarrow \mathbb{C} .
$$

A crucial property of classical interacting fields - which does not hold anymore for interacting quantum fields - is their factorization with respect to the classical product

$$
(F \cdot G)_{S_{0}, S}^{\mathrm{ret}}=F_{S_{0}, S}^{\mathrm{ret}} \cdot G_{S_{0}, S}^{\mathrm{ret}} .
$$

This is why certain symmetry properties of classical field theory in general cannot be transferred directly into quantum field theory (see Sect. 5.1).

In classical field theory retarded products $R_{\mathrm{cl}}$ are defined as coefficients in the expansion (with respect to the interaction) of interacting retarded fields [1]:

$$
R_{\mathrm{cl}}: \mathcal{F}(\mathcal{C})^{\otimes n} \otimes \mathcal{F}(\mathcal{C}) \rightarrow \mathcal{F}(\mathcal{C}),\left.\quad R_{\mathrm{cl}}\left(S^{\otimes n}, F\right) \stackrel{\text { def }}{=} \frac{d^{n}}{d \lambda^{n}}\right|_{\lambda=0} F \circ r_{S_{0}+\lambda S, S_{0}} .
$$

Interacting fields can then be written as

$$
F_{S_{0}, S}^{\mathrm{ret}} \simeq \sum_{n=0}^{\infty} \frac{1}{n !} R_{\mathrm{cl}}\left(S^{\otimes n}, F\right) \equiv R_{\mathrm{cl}}\left(e_{\otimes}^{S}, F\right)
$$

where the r.h.s. of $\simeq$ is interpreted as a formal power series in $S$ (we do not care about convergence of the series). In the last expression $R_{\mathrm{cl}}$ is viewed as a linear map

$$
R_{\mathrm{cl}}: \mathbb{T} \mathcal{F}(\mathcal{C}) \otimes \mathcal{F}(\mathcal{C}) \rightarrow \mathcal{F}(\mathcal{C}),
$$

where $\mathbb{T} \mathcal{V} \stackrel{\text { def }}{=} \mathbb{C} \oplus \bigoplus_{n=1}^{\infty} \mathcal{V}^{\otimes n}$ denotes the tensor algebra corresponding to some vector space $\mathcal{V}$.

By introducing the differential operator

$$
\mathcal{D}_{S_{0}, S}(\lambda) \stackrel{\text { def }}{=}-\int d x d y \Delta_{S_{0}+\lambda S}^{\mathrm{ret}}(x, y) \frac{\delta S}{\delta \varphi(y)} \frac{\delta}{\delta \varphi(x)},
$$

we obtain from (2.12) the following explicit expression for the interacting field:

$$
F_{S_{0}, S}^{\mathrm{ret}} \simeq F+\sum_{n=1}^{\infty} \int_{0}^{1} d \lambda_{1} \int_{0}^{\lambda_{1}} d \lambda_{2} \ldots \int_{0}^{\lambda_{n-1}} d \lambda_{n} \mathcal{D}_{S_{0}, S}\left(\lambda_{n}\right) \ldots \mathcal{D}_{S_{0}, S}\left(\lambda_{1}\right) F .
$$

To first order in $S$ this formula reads (see also [1])

$$
R_{\mathrm{cl}}(S, F)=-\int d x d y \Delta_{S_{0}}^{\mathrm{ret}}(x, y) \frac{\delta S}{\delta \varphi(y)} \frac{\delta F}{\delta \varphi(x)} .
$$

We can now endow classical fields with a Poisson structure: we introduce the (offshell) Poisson bracket using Peierls definition [21] (see also [22] and [1]) 
Definition 2. The Poisson bracket associated to the action $S \in \mathcal{F}(\mathcal{C})$ is the map

$$
\begin{gathered}
\{\cdot, \cdot\}_{S}: \mathcal{F}(\mathcal{C}) \otimes \mathcal{F}(\mathcal{C}) \rightarrow \mathcal{F}(\mathcal{C}) \\
\{F, G\}_{S} \stackrel{\text { def }}{=} R_{S}(F, G)-R_{S}(G, F) .
\end{gathered}
$$

where

$$
\left.R_{S}(F, G) \stackrel{\text { def }}{=} \frac{d}{d \lambda}\right|_{\lambda=0} G \circ r_{S+\lambda F, S}
$$

The properties

- $R_{S}(F, G)=-\int d x d y \frac{\delta G}{\delta \varphi(x)} \Delta_{S}^{\mathrm{ret}}(x, y) \frac{\delta F}{\delta \varphi(y)}$ (which is a generalization of (2.22)) and

- $\{\cdot, \cdot\}_{S}$ is indeed a Poisson bracket, i.e. it satisfies the Leibniz rule and the Jacobi identity,

are proved in [1] for the on-shell restrictions $R_{S}^{\text {on-shell }}(F, G)=\left.R_{S}(F, G)\right|_{\mathcal{C}_{S}}$ and $\{F, G\}_{S}^{\text {on-shell }}=\left.\{F, G\}_{S}\right|_{\mathcal{C}_{S}}$. These proofs can easily be generalized to $R_{S}$ and $\{\cdot, \cdot\}_{S}$. The Jacobi identity is derived from

$$
\left\{R_{S}(H, F), G\right\}_{S}+\left\{F, R_{S}(H, G)\right\}_{S}=R_{S}\left(H,\{F, G\}_{S}\right)+\left.\frac{d}{d \lambda}\right|_{\lambda=0}\{F, G\}_{S+\lambda H},
$$

which is the infinitesimal version of the statement that the map $\mathcal{F}(\mathcal{C}) \ni F \mapsto F \circ$ $r_{S_{1}, S_{2}} \in \mathcal{F}(\mathcal{C})$ is a canonical transformation:

$$
\left\{F \circ r_{S_{1}, S_{2}}, G \circ r_{S_{1}, S_{2}}\right\}_{S_{2}}=\{F, G\}_{S_{1}} \circ r_{S_{1}, S_{2}} .
$$

In [1] only the proof of the infinitesimal version (2.26) is given. We are going to show that integration of $(2.26)$, written in a suitable form, yields indeed $(2.27) .{ }^{5}$ First note

$$
\left.\frac{d}{d \lambda}\right|_{\lambda=0} F \circ r_{S, S+\lambda H}=-\left.\frac{d}{d \lambda}\right|_{\lambda=0} F \circ r_{S+\lambda H, S}=-R_{S}(H, F) .
$$

Let $H:=S_{2}-S_{1}, S(\lambda):=S_{1}+\lambda H$ and $F_{\lambda}:=F \circ r_{S_{1}, S(\lambda)}$. With that we obtain

$$
\begin{gathered}
\left.\frac{d}{d \lambda}\right|_{\lambda=\lambda_{0}}\left\{F \circ r_{S_{1}, S(\lambda)}, G \circ r_{S_{1}, S(\lambda)}\right\}_{S(\lambda)} \circ r_{S(\lambda), S_{1}}= \\
\left.\frac{d}{d \lambda}\right|_{\lambda=\lambda_{0}}\left(\left\{F_{\lambda_{0}} \circ r_{S\left(\lambda_{0}\right), S(\lambda)}, G_{\lambda_{0}} \circ r_{S\left(\lambda_{0}\right), S(\lambda)}\right\}_{S(\lambda)} \circ r_{S(\lambda), S\left(\lambda_{0}\right)}\right) \circ r_{S\left(\lambda_{0}\right), S_{1}}=0
\end{gathered}
$$

by using (2.25), (2.28) and the infinitesimal version (2.26). Integrating this equation over $\lambda_{0}$ from $\lambda_{0}=0$ to $\lambda_{0}=1$ it results the assertion.

Due to the perturbative expansion around the free theory only the Poisson bracket associated to the free action, $\{\cdot, \cdot\}_{\mathrm{cl}} \equiv\{\cdot, \cdot\}_{S_{0}}$, will be used in the following sections.

As one can easily check, the retarded products (2.17) have the same properties as the on-shell retarded products in [1] (which are related to $(2.17)$ by $R_{\mathrm{cl}}^{\text {on-shell }}\left(S^{\otimes n}, F\right)=$ $\left.\left.R_{\mathrm{cl}}\left(S^{\otimes n}, F\right)\right|_{\mathcal{C}_{S_{0}}}\right)$. These are the properties which are used to define retarded products in perturbative QFT in an axiomatic way.

\footnotetext{
${ }^{5}$ This proof is due to Klaus Fredenhagen.
} 


\section{Perturbative Quantum Field Theory}

We summarize the quantization of perturbative classical fields as it is worked out in [16] on the basis of causal perturbation theory [17], [18] and work of Steinmann [23]. Since the direct quantization of an interacting theory is in general not solved, we quantize the free theory, around which the perturbative expansion is done (see Sect. 2), by using deformation quantization: we replace $\mathcal{F}(\mathcal{C})$ and $\mathcal{F}_{\text {loc }}(\mathcal{C})$ by $\mathcal{F} \stackrel{\text { def }}{=} \mathcal{F}(\mathcal{C})[[\hbar]]$ and $\mathcal{F}_{\text {loc }} \stackrel{\text { def }}{=} \mathcal{F}_{\text {loc }}(\mathcal{C})[[\hbar]]$ resp. (i.e. all functionals are formal power series in $\hbar$ ) and deform the classical product into the $\star$-product, $\star: \mathcal{F} \times \mathcal{F} \rightarrow \mathcal{F}$, which is still associative but non-commutative and is defined by

$$
\begin{aligned}
(F \star G)(\varphi):= & \sum_{n=0}^{\infty} \frac{\hbar^{n}}{n !} \int d x_{1} \ldots d x_{n} d y_{1} \ldots d y_{n} \frac{\delta^{n} F}{\delta \varphi\left(x_{1}\right) \cdots \delta \varphi\left(x_{n}\right)} \\
& \cdot \prod_{i=1}^{n} H_{m}\left(x_{i}-y_{i}\right) \frac{\delta^{n} G}{\delta \varphi\left(y_{1}\right) \cdots \delta \varphi\left(y_{n}\right)} .
\end{aligned}
$$

There is a freedom in the choice of the 2-point function $H_{m}(x)$ : it is required to differ from the Wightman 2-point function $\Delta_{m}^{+}(x)$ by a smooth and even function of $x$, to be Lorentz invariant and to satisfy the Klein Gordon equation. The 'vacuum state' is the map (see (2.2))

$$
\omega_{0}: \mathcal{F} \longrightarrow \mathbb{C}[[\hbar]], F \mapsto F(0)=f_{0} .
$$

For the interacting quantum field $F_{G},\left(F, G \in \mathcal{F}_{\text {loc }}\right)$ one makes the ansatz ${ }^{6}$ of a formal power series in the interaction $G$ :

$$
F_{G}=\sum_{n=0}^{\infty} \frac{1}{n !} R_{n, 1}\left(G^{\otimes n}, F\right) \equiv R\left(e_{\otimes}^{G}, F\right) .
$$

The 'retarded product' $R_{n, 1}$ is a linear map, from $\mathcal{F}_{\text {loc }}^{\otimes n} \otimes \mathcal{F}_{\text {loc }}$ into $\mathcal{F}$ which is symmetric in the first $n$ variables. The last expression in (3.3) is understood analogously to (2.19). We interpret $R\left(A_{1}\left(x_{1}\right), \ldots ; A_{n}\left(x_{n}\right)\right), A_{1}, \ldots, A_{n} \in \mathcal{P}$, as $\mathcal{F}$-valued distributions on $\mathcal{D}\left(\mathbb{M}^{n}\right)$, which are defined by: $\int d x h(x) R(\ldots, A(x), \ldots):=R(\ldots \otimes A(h) \otimes \ldots) \forall h \in$ $\mathcal{D}(\mathbb{M})$.

Interacting fields are defined by the following axioms [16], which are motivated by the principle that we want to maintain as much as possible of the classical structure in the process of quantization:

\section{Basic axioms:}

Initial Condition. $R(F) \equiv R_{0,1}(1, F)=F$.

Causality. $F_{G+H}=F_{G}$ if $\operatorname{supp}\left(\frac{\delta F}{\delta \varphi}\right) \cap\left(\operatorname{supp}\left(\frac{\delta H}{\delta \varphi}\right)+\bar{V}_{+}\right)=\emptyset$.

\footnotetext{
${ }^{6}$ With respect to factors of $\hbar$, our conventions $(R)$ differ from [16] $\left(R^{D F}\right)$, namely $R\left(e_{\otimes}^{G}, F\right)=$ $R^{D F}\left(e_{\otimes}^{G / \hbar}, F\right)$. However, for the $T$-products we use the same conventions.
} 
GLZ Relation. In the classical GLZ Relation we replace the Poisson bracket $\{\cdot, \cdot\}_{\mathrm{cl}}$ by

$$
\{\cdot, \cdot\} \stackrel{\text { def }}{=} \frac{1}{i \hbar}[\cdot, \cdot]_{\star}=\{\cdot, \cdot\}_{\mathrm{cl}}+\mathcal{O}(\hbar)
$$

(where $[H, F]_{\star} \equiv H \star F-F \star H$ ). This gives

$$
\left\{F_{G}, H_{G}\right\}=\left.\frac{d}{d \lambda}\right|_{\lambda=0}\left(F_{G+\lambda H}-H_{G+\lambda F}\right) .
$$

Based on these requirements, the retarded products $R_{n, 1}$ can be constructed by induction on $n$. However, in each inductive step one is free to add a local functional, which corresponds to the usual renormalization ambiguity. This ambiguity is reduced by imposing the following further axioms:

\section{Renormalization conditions:}

Unitarity. $\left(F_{G}\right)^{*}=F_{G^{*}}^{*}$

Poincare Covariance. For $L \in \mathcal{P}_{+}^{\uparrow}$ we set $\varphi_{L}(x):=\varphi\left(L^{-1} x\right)$ and $h_{L}(x):=h\left(L^{-1} x\right)$, $h \in \mathcal{C}$, and define an automorphism

$$
\beta_{L}: \mathcal{F} \rightarrow \mathcal{F} ; \beta_{L}\left(\left\langle f_{n}, \varphi^{\otimes n}\right\rangle\right)=\left\langle f_{n},\left(\varphi_{L^{-1}}\right)^{\otimes n}\right\rangle,
$$

that is $\left(\beta_{L} F\right)(h)=F\left(h_{L^{-1}}\right) . \mathcal{P}_{+}^{\uparrow}$-Covariance of the interacting fields means:

$\beta_{L}\left(F_{G}\right)=\left(\beta_{L} F\right)_{\beta_{L} G}, \forall L \in \mathcal{P}_{+}^{\uparrow}$.

Field Independence. The interacting field $F_{G}$ depends on $\varphi$ only through $F$ and $G: \frac{\delta R}{\delta \varphi(x)}=0$. This condition is equivalent to the requirement that $R$ fulfills the causal Wick expansion [18], that is

$$
\begin{gathered}
R_{n-1,1}\left(A_{1}\left(x_{1}\right) \otimes \cdots \otimes A_{n-1}\left(x_{n-1}\right), A_{n}\left(x_{n}\right)\right)=\sum_{l_{1}, \ldots, l_{n}} \frac{1}{l_{1} ! \cdots l_{n} !} \\
\times \omega_{0}\left(R_{n-1,1}\left(\cdots \sum_{a_{i 1} \ldots a_{i l_{i}}} \frac{\partial^{l_{i}} A_{i}}{\partial\left(\partial^{a_{i 1}} \varphi\right) \cdots \partial\left(\partial^{a_{i l_{i}}} \varphi\right)}\left(x_{i}\right) \cdots\right)\right) \prod_{i=1}^{n} \prod_{j_{i}=1}^{l_{i}} \partial^{a_{i j_{i}}} \varphi\left(x_{i}\right)
\end{gathered}
$$

with multi-indices $a_{i j_{i}} \in \mathbb{N}_{0}^{d}$.

Field Equation.

$$
\varphi_{G}(x)=\varphi(x)-\int \Delta_{m}^{\mathrm{ret}}(x-y)\left(\frac{\delta G}{\delta \varphi(y)}\right)_{G} d y \quad, \quad \forall G \in \mathcal{F}_{\mathrm{loc}} .
$$

Smoothness in $m$. Through the GLZ condition the interacting fields depend on the 2-point function $H_{m}$ and with that they depend on the mass $m$ of the free field: $F_{G} \equiv\left(F_{G}\right)^{H_{m}}$. We require that the maps

$$
0 \leq m \mapsto\left(F_{G}\right)^{H_{m}}, \quad F, G \in \mathcal{F}_{\text {loc }},
$$


are smooth. In even dimensional spacetime this excludes the 2-point function $\Delta_{m}^{+}$ due to logarithmic singularities at $m=0$; more generally, homogeneous scaling of $H_{m}$ is not compatible with smoothness in $m \geq 0$. As in [16] we work with the 2-point function $H_{m}^{\mu}$ (and the corresponding Feynman propagator) which is distinguished by almost homogeneous scaling [19]. $H_{m}^{\mu}$ depends on an additional mass parameter $\mu>0$ and is explicitly given in Appendix A of [16]. For the corresponding star product, retarded product and interacting fields we write $\star_{m, \mu}, R^{(m, \mu)}$ and $\left(F_{G}\right)^{(m, \mu)} \equiv\left(F_{G}\right)^{H_{m}^{\mu}}$, respectively.

$\mu$-Covariance. The $\star$-products $\left(\star_{m, \mu}\right)_{\mu>0}$ (and the $\star$-product with respect to $\left.\Delta_{m}^{+}\right)$are equivalent, that is there exists an invertible operator $\left(\mu_{2} / \mu_{1}\right)^{\Gamma}$ which intertwines these products (see e.g. [14]):

$$
F \star_{m, \mu_{2}} G=\left(\mu_{2} / \mu_{1}\right)^{\Gamma^{(m)}}\left(\left(\left(\mu_{2} / \mu_{1}\right)^{-\Gamma^{(m)}} F\right) \star_{m, \mu_{1}}\left(\left(\mu_{2} / \mu_{1}\right)^{-\Gamma^{(m)}} G\right)\right),
$$

where $r^{\Gamma \stackrel{\text { def }}{=}} 1+\sum_{k=1}^{\infty} \frac{1}{k !}(\log (r) \cdot \Gamma)^{k}($ for $r>0)$ and

$$
\Gamma \equiv \Gamma^{(m)} \stackrel{\text { def }}{=} \int d x d y m^{d-2} f\left(m^{2}(x-y)^{2}\right) \frac{\delta^{2}}{\delta \varphi(x) \delta \varphi(y)} .
$$

(The smooth function $f$ is explicitly given by formula (A.9) in [16].) The axiom $\mu$-Covariance requires that $\left(\mu_{2} / \mu_{1}\right)^{\Gamma}$ intertwines also the retarded products: ${ }^{7}$

$$
R^{\left(m, \mu_{2}\right)}=\left(\mu_{2} / \mu_{1}\right)^{\Gamma^{(m)}} \circ R^{\left(m, \mu_{1}\right)} \circ \mathbb{T}\left(\mu_{2} / \mu_{1}\right)^{-\Gamma^{(m)}} .
$$

Scaling. The mass dimension of a monomial in $\mathcal{P}$ is defined by the conditions

$$
\operatorname{dim}\left(\partial^{a} \varphi\right)=\frac{d-2}{2}+|a| \text { and } \operatorname{dim}\left(A_{1} A_{2}\right)=\operatorname{dim}\left(A_{1}\right)+\operatorname{dim}\left(A_{2}\right)
$$

for all monomials $A_{1}, A_{2} \in \mathcal{P}$. The mass dimension of a polynomial in $\mathcal{P}$ is the maximum of the mass dimensions of the contributing monomials. We denote by $\mathcal{P}_{\text {hom }}$ the set of all field polynomials which are homogeneous in the mass dimension. A scaling transformation $\sigma_{\rho}$ is introduced as an automorphism of $\mathcal{F}$ (considered as an algebra with the classical product) by

$$
\sigma_{\rho}\left(\left\langle f_{n}, \varphi^{\otimes n}\right\rangle\right) \stackrel{\text { def }}{=} \rho^{\frac{n(2-d)}{2}} \int d x_{1} \ldots d x_{n} f_{n}\left(x_{1}, \ldots, x_{n}\right) \varphi\left(x_{1} / \rho\right) \ldots \varphi\left(x_{n} / \rho\right) .
$$

For $A \in \mathcal{P}_{\text {hom }}$ we obtain $\rho^{\operatorname{dim}(A)} \sigma_{\rho}(A(\rho x))=A(x)$. Our condition of almost homogeneous scaling states that

$$
\sigma_{\rho} \circ R_{n, 1}^{\left(\rho^{-1} m, \mu\right)} \circ\left(\sigma_{\rho}^{-1}\right)^{\otimes(n+1)}, \quad n \in \mathbb{N}_{0}, \quad m \geq 0, \quad \mu>0,
$$

is a polynomial in $(\log \rho)$.

\footnotetext{
${ }^{7}$ Given a linear map $f: \mathcal{V} \rightarrow \mathcal{V}$ (where $\mathcal{V}$ is a vector space) we define$$
\mathbb{T} f: \mathbb{T} \mathcal{V} \rightarrow \mathbb{T} \mathcal{V} ;(\mathbb{T} f)\left(c \oplus \bigoplus_{j=1}^{\infty}\left(v_{j 1} \otimes \ldots \otimes v_{j j}\right)\right)=c \oplus \bigoplus_{j=1}^{\infty}\left(f\left(v_{j 1}\right) \otimes \ldots \otimes f\left(v_{j j}\right)\right)
$$ 
The construction of the retarded products proceeds in terms of the distributions $R\left(A_{1}\left(x_{1}\right), \ldots ; A_{n}\left(x_{n}\right)\right), A_{1}, \ldots, A_{n} \in \mathcal{P}$. Since the retarded products depend only on the functionals (and not on how the latter are written as smeared fields (2.4)), they must satisfy the Action Ward Identity (AWI) [16]:

$$
\partial_{\mu}^{x} R_{n-1,1}\left(\ldots A_{k}(x) \ldots\right)=R_{n-1,1}\left(\ldots, \partial_{\mu} A_{k}(x), \ldots\right) .
$$

The AWI can simply be fulfilled by constructing $R\left(A_{1}\left(x_{1}\right), \ldots ; A_{n}\left(x_{n}\right)\right)$ first only for balanced fields $A_{k} \in \mathcal{P}_{\text {bal }} \forall k$, and by using the AWI and linearity for the extension to general fields $A_{k} \in \mathcal{P}$.

The axioms Smoothness in $m, \mu$-Covariance and Scaling can be replaced by the weaker axiom Scaling Degree, which requires that 'renormalization may not make the interacting fields more singular' (in the UV-region). Usually this is formulated in terms of Steinmann's scaling degree [24]:

$$
\operatorname{sd}(f) \stackrel{\text { def }}{=} \inf \left\{\delta \in \mathbb{R} \mid \lim _{\rho \downarrow 0} \rho^{\delta} f(\rho x)=0\right\}, \quad f \in \mathcal{D}^{\prime}\left(\mathbb{R}^{k}\right) \text { or } f \in \mathcal{D}^{\prime}\left(\mathbb{R}^{k} \backslash\{0\}\right) .
$$

Namely, one requires

$$
\operatorname{sd}\left(\omega_{0}\left(R\left(A_{1}, \ldots ; A_{n}\right)\right)\left(x_{1}-x_{n}, \ldots\right)\right) \leq \sum_{j=1}^{n} \operatorname{dim}\left(A_{j}\right), \quad \forall A_{j} \in \mathcal{P}_{\text {hom }},
$$

where Translation Invariance is assumed.

In the inductive construction of the sequence $\left(R_{n, 1}\right)_{n \in \mathbb{N}}$ (given in [16]), the problem of renormalization appears as the extension of $\mathbb{C}[[\hbar]]$-valued distributions from $\mathcal{D}\left(\mathbb{R}^{d n} \backslash\right.$ $\{0\})$ to $\mathcal{D}\left(\mathbb{R}^{d n}\right)$. This extension has to be done in the sense of formal power series in $\hbar$, that is individually in each order in $\hbar$. With that it holds

$$
\lim _{\hbar \rightarrow 0} R=R_{\mathrm{cl}} .
$$

Namely, the GLZ Relation is the only axiom which depends explicitly on $\hbar$ and in the classical limit it goes over into the classical GLZ Relation, due to (3.4).

The retarded product, having two different kinds of arguments, can be derived from the time ordered product ('T-product') $T: \mathbb{T} \mathcal{F}_{\text {loc }} \rightarrow \mathcal{F}$, which is totally symmetric i.e. it has only one kind of arguments. The corresponding relation is Bogoliubov's formula:

$$
R\left(e_{\otimes}^{S}, F\right)=-\left.i \hbar \mathbf{S}(S)^{-1} \star \frac{d}{d \tau}\right|_{\tau=0} \mathbf{S}(S+\tau F), \quad \mathbf{S}(S) \equiv T\left(e_{\otimes}^{i S / \hbar}\right),
$$

The axioms for retarded products translate directly into corresponding axioms for $T$-products, see Appendix E of [16]. There is no axiom corresponding to the GLZ Relation. The latter can be interpreted as 'integrability condition' for the 'vector potential' $R\left(e_{\otimes}^{S}, F\right)$, that is it ensures the existence of the 'potential' $\mathbf{S}(S)$ fulfilling (3.21); for details see [19]. (A derivation of the GLZ Relation from (3.21) is given in [13].)

In [16] it is shown that there exist retarded products which fulfill all axioms. The non-uniqueness of solutions is characterized by the 'Main Theorem'; we use the version given in [16]: 
Theorem 3. (a) Let $R$ and $\hat{R}$ be retarded products which fulfill the basic axioms and the renormalization conditions Unitarity, $\mathcal{P}_{+}^{\uparrow}$-Covariance, Field Independence and Field Equation. Then there exists a unique, symmetric and linear map

$$
D: \mathbb{T} \mathcal{F}_{\text {loc }} \longrightarrow \mathcal{F}_{\text {loc }}
$$

with $D(1)=0, D(F)=F\left(\forall F \in \mathcal{F}_{\text {loc }}\right)$, such that for all $F, S \in \mathcal{F}_{\text {loc }}$ the following intertwining relation holds (in the sense of formal power series in $\lambda$ )

$$
\hat{R}\left(e_{\otimes}^{\lambda S}, F\right)=R\left(e_{\otimes}^{D\left(e_{\otimes}^{\lambda S}\right)}, D\left(e_{\otimes}^{\lambda S} \otimes F\right)\right) .
$$

In addition, $D$ satisfies the conditions:

(i) $\operatorname{supp}\left(\frac{\delta D\left(F_{1} \otimes \cdots \otimes F_{n}\right)}{\delta \varphi}\right) \subset \bigcap_{i \in \underline{n}} \operatorname{supp}\left(\frac{\delta F_{i}}{\delta \varphi}\right), \quad F_{i} \in \mathcal{F}_{\text {loc }}$

(ii) $\frac{\delta D\left(e_{\otimes}^{F}\right)}{\delta \varphi}=D\left(e_{\otimes}^{F} \otimes \frac{\delta F}{\delta \varphi}\right)$

(iii) $D\left(e_{\otimes}^{F} \otimes \varphi(h)\right)=\varphi(h), \quad \forall F \in \mathcal{F}_{\text {loc }}$

(iv) $D\left(F^{\otimes n}\right)^{*}=D\left(\left(F^{*}\right)^{\otimes n}\right)$

(v) $\beta_{L} \circ D=D \circ \mathbb{T} \beta_{L}, \quad \forall L \in \mathcal{P}_{+}^{\uparrow}$

(vi) (A) If $R^{(m, \mu)}, \hat{R}^{(m, \mu)}$ are smooth in $m \geq 0$ and satisfy the axioms $\mu$-Covariance and Scaling, then the corresponding $D^{(m, \mu)}$ is also smooth in $m$, invariant under scaling

$$
\sigma_{\rho} \circ D^{\left(\rho^{-1} m, \mu\right)} \circ \mathbb{T} \sigma_{\rho}^{-1}=D^{(m, \mu)} .
$$

and $\mu$-covariant ${ }^{8}$

$$
D^{\left(m, \mu_{2}\right)}=\left(\mu_{2} / \mu_{1}\right)^{\Gamma^{(m)}} \circ D^{\left(m, \mu_{1}\right)} \circ \mathbb{T}\left(\mu_{2} / \mu_{1}\right)^{-\Gamma^{(m)}} .
$$

(B) Alternatively, if $R$ and $\hat{R}$ satisfy the axiom Scaling degree, then

$$
\operatorname{sd}\left(\omega_{0}\left(D\left(A_{1}, \ldots, A_{n}\right)\right)\left(x_{1}-x_{n}, \ldots\right)\right) \leq \sum_{j=1}^{n} \operatorname{dim}\left(A_{j}\right), \quad \forall A_{j} \in \mathcal{P}_{\text {hom }} .
$$

(b)Conversely, given $R$ and $D$ as above, equation (3.23) gives a new retarded product $\hat{R}$ which satisfies the axioms.

Since the classical limit of the axioms has a unique solution (which is $R_{\mathrm{cl}}$ ), the map $D$ is trivial to lowest order in $\hbar$, i.e.

$$
D\left(e_{\otimes}^{S}\right)=S+\mathcal{O}(\hbar) \quad \text { and } \quad D\left(e_{\otimes}^{S} \otimes F\right)=F+\mathcal{O}(\hbar) .
$$

The relation (3.23) can equivalently be expressed in terms of time ordered products,

$$
\hat{T}\left(e_{\otimes}^{i S / \hbar}\right)=T\left(e_{\otimes}^{i D\left(e_{\otimes}^{S}\right) / \hbar}\right)
$$

where $T$ and $\hat{T}$ are the time ordered products belonging to $R$ and $\hat{R}$, resp., according to $(3.21)$.

\footnotetext{
${ }^{8}$ The claim in [16] that $D^{(m, \mu)}$ is independent of $\mu$, is wrong.
} 


\section{Proper vertices for $T$-products}

Proper vertices ${ }^{9}$ are an old and standard tool in perturbative QFT. In terms of $R$ products the basic idea is the following: a perturbative QFT can be rewritten as classical field theory with a non-local interaction ('proper interaction') which agrees to lowest order in $\hbar$ with the original local interaction. Since $R_{\mathrm{cl}}$ is the sum of all (connected) tree diagrams (as explained in Appendix A), this rewriting means that we interpret each diagram as tree diagram with non-local vertices ('proper vertices') given by the 1-particle-irreducible (1PI) subdiagrams. This structural decomposition of Feynman diagrams can just as well be done for $T$-products and it is this latter form of proper vertices which is well known in the literature. Since $T$-products are totally symmetric, it is simpler to introduce proper vertices in terms of $T$-products than in terms of $R$-products and, hence, we work with the former (for the introduction of proper vertices for $R$-products see Appendix A).

A main motivation to introduce proper vertices is that the renormalization of an arbitrary diagram reduces to the renormalization of its 1PI-subdiagrams. Indeed, due to the validity of the MWI for tree diagrams (i.e. in classical FT), the MWI can equivalently be formulated in terms of proper vertices (i.e. in terms of 1PI-diagrams), see Sect. 5.3. This 'proper MWI' formally coincides with the usual formulation of Ward identities in the functional approach to QFT (for an overview see e.g. [10]).

\subsection{Diagrammatics and definition of the 1-particle-irreducible part $T^{1 \mathrm{PI}}$ of the time ordered product}

To introduce proper vertices we need the tree part $T_{\text {tree }}$ for non-local entries and, for later purpose, the 1PI part $T^{1 \mathrm{PI}}$ of the time ordered product $T$. The definition of $T_{\text {tree }}$ can obviously be given in terms of Feynman diagrams; but in case of $T^{1 \mathrm{PI}}$ we are faced with the problem that for loop diagrams the decomposition of $T\left(A_{1}\left(x_{1}\right), \ldots\right)$ into contributions of Feynman diagrams is non-unique, due to the local terms coming from renormalization.

To motivate the definition of $T^{1 \mathrm{PI}}$ we first study a 'smooth and symmetric $\star$ product': let $f \in \mathcal{C}^{\infty}\left(\mathbb{R}^{4}, \mathbb{C}\right)$ with $f(x)=f(-x), \forall x$. We define $\star_{f}: \mathcal{F} \otimes \mathcal{F} \rightarrow \mathcal{F}$ by replacing in the definition (3.1) of the $\star$-product the 2-point function $H_{m}$ by $f$. This product, $\star_{f}$, is associative and commutative. By definition $\star_{f}$ satisfies 'Wick's theorem'. Due to that, $\star_{f, j=1}^{n} F_{j} \equiv F_{1} \star_{f} \ldots \star_{f} F_{n}$ can uniquely be viewed as a sum of diagrams. In spite of the possible non-locality of the $F_{j}$ 's, we symbolize each $F_{j}$ by one vertex. The contractions are symbolized by inner lines connecting the vertices, which are not oriented due to $f(x)=f(-x)$. (We do not draw any external lines.)

The contribution of the connected diagrams (denoted by $\left.\left(\star_{f, j=1}^{n} F_{j}\right)^{c}\right)$ is obtained from $\star_{f, j=1}^{n} F_{j}$ by subtraction of the contributions of all disconnected diagrams; this

\footnotetext{
${ }^{9}$ In the literature proper vertices (or the 'proper interaction') are sometimes called 'effective vertices' (or 'effective interaction' resp.). However, differently to what we are doing here, the notion 'effective field theory' usually means an approximation to the perturbation series. For this reason we omit the word 'effective' and use the terminology of Sect. 6-2-2 in [25].
} 
gives the recursion relation (see e.g. [15])

$$
\left(\star_{f, j=1}^{n} F_{j}\right)^{c}=\star_{f, j=1}^{n} F_{j}-\sum_{|P| \geq 2} \prod_{J \in P}\left(\star_{f, j \in J} F_{j}\right)^{c},
$$

where the sum runs over all partitions $P$ of $\{1, \ldots, n\}$ in at least two subsets and $\prod$ means the classical product. One easily sees that the linked cluster theorem applies to $\star_{f}$ :

$$
e_{\star_{f}}^{F}=\exp \left(e_{\star_{f}^{c}}^{F}\right), \quad \text { where } \quad e_{\star_{f}}^{F} \stackrel{\text { def }}{=} 1+\sum_{n=1}^{\infty} \frac{F^{\star_{f} n}}{n !}, \quad e_{\star_{f}^{c}}^{F} \stackrel{\text { def }}{=} \sum_{n=1}^{\infty} \frac{\left(F^{\star_{f} n}\right)^{c}}{n !}
$$

(with $F^{\star_{f} n}$ being the $n$-fold product $F \star_{f} \ldots \star_{f} F$ ) and exp. denotes the exponential function with respect to the classical product.

Analogously to (4.1) the contribution of all 1PI-diagrams to $\star_{f, j=1}^{n} F_{j}$ (denoted by $\left.\left(\star_{f, j=1}^{n} F_{j}\right)^{1 \mathrm{PI}}\right)$, is obtained from the connected diagrams $\left(\star_{f, j=1}^{n} F_{j}\right)^{c}$ by subtracting the contributions of all connected one-particle-reducible diagrams. To formulate this we need the contribution of all connected tree diagrams to $\left(\star_{f, j=1}^{n} F_{j}\right)^{c}$, which we denote by $\left(\star_{f, j=1}^{n} F_{j}\right)_{\text {tree }}^{c}$. This diagrammatic definition of $\left(\star_{f, j=1}^{n} F_{j}\right)_{\text {tree }}^{c}$ fulfills the following unique and independent characterizations:

- By recursion: one easily finds that the connected tree diagrams satisfy the recursion relation

$$
\begin{gathered}
\left(\star_{f, j=1}^{n+1} F_{j}\right)_{\mathrm{tree}}^{c}=\sum_{k=1}^{n} \int d x_{1} \ldots d x_{k} d y_{1} \ldots d y_{k} \frac{\delta^{k} F_{n+1}}{\delta \varphi\left(x_{1}\right) \ldots \delta \varphi\left(x_{k}\right)} \\
\prod_{j=1}^{k} f\left(x_{j}-y_{j}\right) \frac{1}{k !} \sum_{I_{1} \sqcup \ldots \sqcup I_{k}=\{1, \ldots, n\}} \frac{\delta}{\delta \varphi\left(y_{1}\right)}\left(\star_{f, j \in I_{1}} F_{j}\right)_{\mathrm{tree}}^{c} \cdot \ldots \\
\frac{\delta}{\delta \varphi\left(y_{k}\right)}\left(\star_{f, j \in I_{k}} F_{j}\right)_{\text {tree }}^{c},
\end{gathered}
$$

where $I_{j} \neq \emptyset \forall j, \sqcup$ means the disjoint union. (Note that in the sum over $I_{1}, \ldots, I_{k}$ the succession of $I_{1}, \ldots, I_{k}$ is distinguished and, hence, there is a factor of $\frac{1}{k !}$.)

- By the power in $\hbar$ : as explained in Sect. 5.2 of [15] it holds

$$
\left(\star_{f, j=1}^{n} F_{j}\right)^{c}=\mathcal{O}\left(\hbar^{n-1}\right) \quad \text { for } F_{1}, \ldots, F_{n} \sim \hbar^{0},
$$

and the contribution of all tree diagrams is given by the terms of lowest order in $\hbar$

$$
\left(\star_{f, j=1}^{n} F_{j}\right)_{\text {tree }}^{c}=\hbar^{n-1} \lim _{\hbar \rightarrow 0} \hbar^{-(n-1)}\left(\star_{f, j=1}^{n} F_{j}\right)^{c} .
$$

The contribution of all tree diagrams to $\star_{f, j=1}^{n} F_{j}$, which we denote by $\left(\star_{f, j=1}^{n} F_{j}\right)_{\text {tree }}$, is related to the connected tree diagrams by the linked cluster theorem: $e_{\star_{f, \text { tree }}}^{F}=$ $\exp \left(e_{\star_{f, \text { tree }}^{c}}^{F}\right)$. (This follows immediately from (4.2) by selecting all tree diagrams.) 
We now give the (above announced) unique recursive characterization of the 1PIdiagrams:

$$
\left(\star_{f, j=1}^{n} F_{j}\right)^{1 \mathrm{PI}}=\left(\star_{f, j=1}^{n} F_{j}\right)^{c}-\sum_{|P| \geq 2}\left(\star_{f, J \in P}\left(\star_{f, j \in J} F_{j}\right)^{1 \mathrm{PI}}\right)_{\text {tree }}^{c} .
$$

The formulas (4.1), (4.4) and (4.5) hold also for the usual $\star$-product (i.e. with $H_{m}$ instead of $f$ ) [15]; but (4.6) needs to be refined, because $H_{m}$ is not symmetrical and, hence, $\star_{H_{m}}$ is not commutative (in particular $\left(F_{1} \star_{H_{m}} \ldots \star_{H_{m}} F_{n}\right)_{\text {tree }}^{c}$ is not symmetrical).

Turning to the time ordered product $T$, we will use (4.1) and (4.6) as motivation for the (recursive) definition of the connected part $T^{c}$ and the 1PI-part $T^{1 \mathrm{PI}}$ of $T$, respectively. So we define [15]

$$
T^{c}\left(\otimes_{j=1}^{n} F_{j}\right) \stackrel{\text { def }}{=} T\left(\otimes_{j=1}^{n} F_{j}\right)-\sum_{|P| \geq 2} \prod_{J \in P} T^{c}\left(\otimes_{j \in J} F_{j}\right) .
$$

It follows that $T$ and $T^{c}$ are related by the linked cluster theorem (4.2): $T\left(e_{\otimes}^{i F}\right)=$ $\exp _{\bullet}\left(T^{c}\left(e_{\otimes}^{i F}\right)\right)$.

The following definition of the connected tree part $T_{\text {tree }}^{c}$ of $T$ applies also to nonlocal entries: ${ }^{10}$

$$
T_{\text {tree }, n}^{c}: \mathcal{F}^{\otimes n} \rightarrow \mathcal{F} ; T_{\text {tree }, n}^{c}\left(\otimes_{j=1}^{n} F_{j}\right)=\left(F_{1} \star_{\Delta^{F}} \ldots \star_{\Delta^{F}} F_{n}\right)_{\text {tree }}^{c},
$$

i.e. we replace in the definition of $\left(\star_{f} \ldots\right)_{\text {tree }}^{c}$ the smooth function $f$ by the Feynman propagator

$$
\begin{aligned}
\Delta^{F}(z) & \equiv \Delta_{m}^{F \mu}(z)=\Theta\left(z^{0}\right) H_{m}^{\mu}(z)+\Theta\left(-z^{0}\right) H_{m}^{\mu}(-z) \\
& =-i \Delta_{m}^{\mathrm{ret}}(z)+H_{m}^{\mu}(-z)=\Delta^{F}(-z)
\end{aligned}
$$

For tree diagrams the resulting expressions are well defined, since pointwise products of Feynman propagators do not appear. Obviously $T_{\text {tree }}^{c}$ fulfills the recursion relation (4.3). In case of local entries another unique characterization of $T_{\text {tree }}^{c}$ is possible: doing renormalization individually in each order in $\hbar, T^{c}$ satisfies $(4.4)$ and $\hbar^{-(n-1)} T_{\text {tree, } n}^{c}$ is the classical limit of $\hbar^{-(n-1)} T_{n}^{c}$ similarly to (4.5). (This is the translation of (3.20) into $T$-products, see Sect. 5.2 of [15].) Analogously one defines the tree part $T_{\text {tree }}$ of $T$ by

$$
T_{\text {tree }, n}\left(\otimes_{j=1}^{n} F_{j}\right) \stackrel{\text { def }}{=}\left(F_{1} \star_{\Delta^{F}} \ldots \star_{\Delta^{F}} F_{n}\right)_{\text {tree }}, \quad F_{j} \in \mathcal{F} \forall j .
$$

\footnotetext{
${ }^{10}$ In QCD the interaction $S=\kappa S_{1}+\kappa^{2} S_{2}$ is a sum of a term of first order in the coupling constant $\kappa, S_{1} \sim \int g A A \partial A(g \in \mathcal{D}(\mathbb{M}, \mathbb{R}))$, and a term of second order in $\kappa, S_{2} \sim \int g^{2} A A A A$. One can achieve that the order in $\kappa$ agrees with the order of the $T$ - (or $R$-)product [26]. Namely, one starts with $T_{1}\left(S_{1}\right)$, the term $S_{2}$ is generated by a non-trivial renormalization of a certain tree diagram: in $T_{2}\left(S_{1}^{\otimes 2}\right) \sim \int d x d y g A A(x) g A A(y) \partial \partial \Delta^{F}(x-y)+\ldots$ the propagator $\partial^{\mu} \partial^{\nu} \Delta^{F}(x-y)$ is replaced by $\partial^{\mu} \partial^{\nu} \Delta^{F}(x-y)-1 / 2 g^{\mu \nu} \delta(x-y)$. Due to the inductive procedure of causal perturbation theory this additional term propagates to higher orders such that this modified $T$-product, $T^{N}$, yields the same $S$-matrix: $T^{N}\left(e_{\otimes}^{i \kappa S_{1}}\right)=T\left(e_{\otimes}^{i\left(\kappa S_{1}+\kappa^{2} S_{2}\right)}\right)$ in the sense of formal power series in $\kappa$. (The corresponding renormalization map $D(3.22)$ is given in [27].) Our definitions of $T_{\text {tree }}^{c}$ (4.8) and $T_{\text {tree }}(4.10)$ do not contain this $1 / 2 g^{\mu \nu} \delta$-term, in agreement with the definition of $R_{\mathrm{cl}}(2.17)$. Generally, in this paper all terms $S_{n}$ of the interaction $S=\sum_{n \geq 1} \kappa^{n} S_{n}$ enter the perturbative construction of the $S$-matrix (or interacting field) already to first order of the $T$ - (or $R$-)product.
} 
Obviously the linked cluster theorem for $\left(\star_{f, \text { tree }}, \star_{f, \text { tree }}^{c}\right)$ is valid also for $f=\Delta^{F}$ : $T_{\text {tree }}\left(e_{\otimes}^{i F}\right)=\exp _{\bullet}\left(T_{\text {tree }}^{c}\left(e_{\otimes}^{i F}\right)\right)$.

Since $T_{\text {tree }}^{c}$ is totally symmetric we may define $T^{1 P I}$ in analogy to (4.6) by the recursive formula

$$
\begin{gathered}
T^{1 P I}\left(F^{\otimes n}\right) \stackrel{\text { def }}{=} T^{c}\left(F^{\otimes n}\right)-\sum_{k=2}^{n} \sum_{l_{1}+\ldots+l_{k}=n, l_{j} \geq 1 \forall j} \frac{n !}{k ! l_{1} ! \ldots l_{k} !} . \\
\cdot T_{\text {tree }, \mathrm{k}}^{c}\left(T^{1 P I}\left(F^{\otimes l_{1}}\right) \otimes \ldots \otimes T^{1 P I}\left(F^{\otimes l_{k}}\right)\right) .
\end{gathered}
$$

The renormalization conditions listed in Sect. 3 are satisfied by $T_{\text {tree }}^{c} \equiv T_{\text {tree }}^{c(m, \mu)}$, $T_{\text {tree }} \equiv T_{\text {tree }}^{(m, \mu)}$ and, provided that $T$ fulfills these conditions, also by $T^{1 P I} \equiv T^{1 P I(m, \mu)}$ (apart from the Field Equation). This can be verified by using the definitions (4.8), (4.10), (4.11) and corresponding properties of the Feynman propagator. Or, in case of local interactions, these properties can be derived for $T_{\text {tree }}^{c}$ by the classical limit of $\hbar^{-(n-1)} T_{n}^{c}$; the linked cluster theorem implies then their validity for $T_{\text {tree }}$.

\subsection{Definition and basic properties of the vertex function $\Gamma_{T}$}

Note that $T$ and $T_{\text {tree }}$ satisfy the relations

$$
T_{\text {(tree) }}(1)=1, \quad T_{(\text {tree })}(F)=F, \quad T_{(\text {tree } \mathrm{n}+1}\left(1 \otimes F_{1} \otimes \ldots\right)=T_{(\text {tree }) \mathrm{n}}\left(F_{1} \otimes \ldots\right),
$$

which imply the following conclusions for $T_{\text {tree }}$ and $T$

$$
\begin{gathered}
T_{\text {(tree) }}\left(e_{\otimes}^{\sum_{n=1}^{\infty} F_{n} \lambda^{n}} \otimes \sum_{n=0}^{\infty} G_{n} \lambda^{n}\right)=0 \Longrightarrow G_{n}=0 \forall n \\
T_{\text {(tree) }}\left(e_{\otimes}^{\sum_{n=1}^{\infty} F_{n} \lambda^{n}}\right)=T_{\text {(tree) }}\left(e_{\otimes}^{\sum_{n=1}^{\infty} G_{n} \lambda^{n}}\right) \Longrightarrow F_{n}=G_{n} \forall n
\end{gathered}
$$

(where $F_{n}, G_{n} \in \mathcal{F}$ are independent of $\lambda$ ), as one obtains by proceeding by induction on the order in $\lambda$.

$R$ - and $T$-products can be obtained from each other by Bogoliubov's formula (3.21), which we will use in the more explicit form

$$
R\left(e_{\otimes}^{S}, F\right)=\bar{T}\left(e_{\otimes}^{-i S / \hbar}\right) \star T\left(e_{\otimes}^{i S / \hbar} \otimes F\right),
$$

where the anti-chronological product $\bar{T}$ is defined by

$$
\bar{T}\left(e_{\otimes}^{-i G}\right) \equiv T\left(e_{\otimes}^{i G}\right)^{-1}=\sum_{n=0}^{\infty}\left(1-T\left(e_{\otimes}^{i G}\right)\right)^{\star n} .
$$

$\left(T\left(e_{\otimes}^{i G}\right)^{-1}\right.$ is the inverse with respect to the $\star$-product.) Although $R$ contains solely connected diagrams (see Appendix A), disconnected diagrams of $T$ and $\bar{T}$ contribute to (4.15). Unitarity reads

$$
\bar{T}_{\text {(tree) }}\left(e_{\otimes}^{-i G}\right)=T_{(\text {tree })}\left(e_{\otimes}^{i G^{*}}\right)^{*}
$$


in this form it holds for the tree diagrams separately, where $\bar{T}_{\text {tree }}$ is defined by (4.10) with $\Delta^{F}$ replaced by the Anti-Feynman propagator $\Delta^{A F}(x)=\Delta^{F}(x)^{*}$. However, note that $T_{\text {tree }}\left(e_{\otimes}^{i G}\right) \star T_{\text {tree }}\left(e_{\otimes}^{i G^{*}}\right)^{*}$ is not equal to 1 .

We define the 'vertex function' $\Gamma_{T}$ implicitly by the following Proposition:

Proposition 4. There exists a totally symmetric and linear map

$$
\Gamma_{T}: \mathbb{T} \mathcal{F}_{\text {loc }} \rightarrow \mathcal{F}
$$

which is uniquely determined by

$$
T\left(e_{\otimes}^{i S / \hbar}\right)=T_{\text {tree }}\left(e_{\otimes}^{i \Gamma_{T}\left(e_{\otimes}^{S}\right) / \hbar}\right) .
$$

To zeroth and first order in $S$ we obtain

$$
\Gamma_{T}(1)=0, \quad \Gamma_{T}(S)=S .
$$

The defining relation (4.19) also implies

$$
T\left(e_{\otimes}^{i S / \hbar} \otimes F\right)=T_{\text {tree }}\left(e_{\otimes}^{i \Gamma_{T}\left(e_{\otimes}^{S}\right) / \hbar} \otimes \Gamma_{T}\left(e_{\otimes}^{S} \otimes F\right)\right) .
$$

For $S=0$ this gives $F=\Gamma_{T}(1 \otimes F)$. The Proposition remains true if, in (4.19), we replace the time ordered product $\left(T, T_{\text {tree }}\right)$ by the anti-chronological product $\left(\bar{T}, \bar{T}_{\text {tree }}\right)$ and $i$ by $(-i)$; we denote the corresponding vertex function by $\bar{\Gamma}_{T}$.

Proof. We construct $\Gamma_{T}\left(\otimes_{j=1}^{n} F_{j}\right)$ by induction on $n$, starting with (4.20). Let $\Gamma_{T}$ of less than $n$ factors be constructed. Then, (4.19) and the requirements total symmetry and linearity determine $\Gamma_{T}\left(\otimes_{j=1}^{n} F_{j}\right)$ uniquely:

$$
\Gamma_{T}\left(\otimes_{j=1}^{n} F_{j}\right)=(i / \hbar)^{n-1} T\left(\otimes_{j=1}^{n} F_{j}\right)-\sum_{|P| \geq 2}(i / \hbar)^{|P|-1} T_{\text {tree }}\left(\bigotimes_{J \in P} \Gamma_{T}\left(\otimes_{j \in J} F_{j}\right)\right),
$$

where $P$ is a partition of $\{1, \ldots, n\}$ in $|P|$ subsets $J$. Obviously the so constructed $\Gamma_{T}$ is totally symmetric and linear.

The proof applies also to the connected parts $\left(T^{c}, T_{\text {tree }}^{c}\right)$. Hence, a totally symmetric and linear map $\Gamma_{c}$ can be defined analogously to (4.18)-(4.19), that is by

$$
T^{c}\left(e_{\otimes}^{i S / \hbar}\right)=T_{\text {tree }}^{c}\left(e_{\otimes}^{i \Gamma_{c}\left(e_{\otimes}^{S}\right) / \hbar}\right) .
$$

The linked cluster theorem for $T$ and $T_{\text {tree }}$ and the definitions of $\Gamma_{T}$ and $\Gamma_{c}$ give

$$
T_{\text {tree }}\left(e_{\otimes}^{i \Gamma_{c}\left(e_{\otimes}^{S}\right) / \hbar}\right)=T_{\text {tree }}\left(e_{\otimes}^{i \Gamma_{T}\left(e_{\otimes}^{S}\right) / \hbar}\right)
$$

and with (4.14) we conclude

$$
\Gamma_{T}=\Gamma_{c}
$$


Therefore, on the right-hand side of (4.22) we may replace the time ordered products $T$ and $T_{\text {tree, } \mathrm{k}}$ by their connected parts:

$$
\begin{gathered}
\Gamma_{T}\left(S^{\otimes n}\right)=(i / \hbar)^{n-1} T^{c}\left(S^{\otimes n}\right)-\sum_{k=2}^{n} \sum_{\substack{l_{1}+\cdots+l_{k}=n \\
l_{j} \geq 1 \forall j}} \frac{(i / \hbar)^{k-1} n !}{k ! l_{1} ! \ldots l_{k} !} . \\
\cdot T_{\text {tree }, \mathrm{k}}^{c}\left(\Gamma_{T}\left(S^{\otimes l_{1}}\right) \otimes \ldots \otimes \Gamma_{T}\left(S^{\otimes l_{k}}\right)\right) .
\end{gathered}
$$

Now let $S \sim \hbar^{0}$ and $F \sim \hbar^{0}$. From (4.26) and $T^{c}\left(S^{\otimes n}\right)-T_{\text {tree }}^{c}\left(S^{\otimes n}\right)=\mathcal{O}\left(\hbar^{n}\right)$ we inductively conclude

$$
\Gamma_{T}\left(e_{\otimes}^{S}\right)=S+\mathcal{O}(\hbar), \quad \Gamma_{T}\left(e_{\otimes}^{S} \otimes F\right)=F+\mathcal{O}(\hbar) .
$$

Motivated by this relation and (4.19) we call $\Gamma_{T}\left(e_{\otimes}^{S}\right)$ the 'proper interaction'.

By comparing the recursion relation (4.26) for $\Gamma_{T}$ with the recursive definition of $T^{1 \mathrm{PI}}$ (4.11) we conclude:

\section{Corollary 5.}

$$
\Gamma_{T}\left(e_{\otimes}^{S}\right)=(\hbar / i) T^{1 \mathrm{PI}}\left(e_{\otimes}^{i S / \hbar}\right) .
$$

Analogously to the Main Theorem it holds:

Lemma 6. The validity of the renormalization conditions for $T\left(\equiv T^{(m, \mu)}\right)$ implies corresponding properties of $\Gamma_{T}\left(\equiv \Gamma_{T}^{(m, \mu)}\right)$ :

- $\mathcal{P}_{+}^{\uparrow}$-Covariance: $\beta_{L} \circ \Gamma_{T}=\Gamma_{T} \circ \mathbb{T} \beta_{L}$ for all $L \in \mathcal{P}_{+}^{\uparrow}$;

- Unitarity: $\Gamma_{T}\left(e_{\otimes}^{S}\right)^{\star}=\bar{\Gamma}_{T}\left(e_{\otimes}^{S^{\star}}\right)$;

- Field Independence: $\frac{\delta \Gamma_{T}\left(e_{\otimes}^{S}\right)}{\delta \varphi}=\Gamma_{T}\left(\frac{\delta S}{\delta \varphi} \otimes e_{\otimes}^{S}\right)$;

- Field Equation: $\Gamma_{T}\left(e_{\otimes}^{S} \otimes \varphi(h)\right)=\varphi(h)$;

- Smoothness in $m \geq 0: \Gamma_{T}^{(m, \mu)}$ is smooth in $m \geq 0$;

- $\mu$-Covariance: $\quad \Gamma_{T}^{\left(m, \mu_{2}\right)}=\left(\mu_{2} / \mu_{1}\right)^{\Gamma} \circ \Gamma_{T}^{\left(m, \mu_{1}\right)} \circ \mathbb{T}\left(\mu_{2} / \mu_{1}\right)^{-\Gamma}$;

- Almost homogeneous Scaling: In contrast to the map D of the Main Theorem, $\Gamma_{T}$ scales only almost homogeneously; $\sigma_{\rho} \circ \Gamma_{T}^{\left(\rho^{-1} m, \mu\right)} \circ \mathbb{T} \sigma_{\rho}^{-1}=\Gamma_{T}^{(m, \mu)}+$ $\mathcal{O}(\log \rho)$ is a polynomial in $\log \rho$.

- If, instead of Smoothness in m, $\mu$-Covariance and Almost homogeneous Scaling, $T$ satisfies the axiom Scaling Degree, then

$$
\operatorname{sd}\left(\omega_{0}\left(\Gamma_{T}\left(A_{1}, \ldots, A_{n}\right)\right)\left(x_{1}-x_{n}, \ldots\right)\right) \leq \sum_{j=1}^{n} \operatorname{dim}\left(A_{j}\right), \quad \forall A_{j} \in \mathcal{P}_{\mathrm{hom}} .
$$


Proof. Each property can be proved for $\Gamma_{T}\left(S^{\otimes n}\right)$ (or $\Gamma_{T}\left(S^{\otimes n} \otimes \varphi(h)\right)$ resp.) by induction on $n$ : we work with the recursion relation (4.22) and use that $T$ and $T_{\text {tree }}$ satisfy the corresponding axiom. In case of the property Scaling Degree we take into account that $\omega_{0}\left(T_{\text {tree }}(\ldots)\right)$ is a tensor product of distributions $t_{j}$ and apply $\operatorname{sd}\left(\otimes_{j} t_{j}\right)=\sum_{j} \operatorname{sd}\left(t_{j}\right)$.

Only the Field Equation is somewhat more involved. We use that $T$ and $T_{\text {tree }}$ fulfil the Field Equation and the Field Independence. This implies

$$
T^{c}\left(\varphi(h) \otimes F_{1} \otimes \ldots \otimes F_{n}\right)=\hbar \int d x d y h(x) \Delta^{F}(x-y) \frac{\delta}{\delta \varphi(y)} T^{c}\left(F_{1} \otimes \ldots \otimes F_{n}\right)
$$

and the same equation for $T_{\text {tree }}^{c}$. In the latter case $F_{1}, \ldots, F_{n}$ may be non-local. (It is not necessary to work with the connected parts, but this simplifies the formulas.) With the recursion relation (4.26) and the inductive assumption we obtain

$$
\begin{aligned}
& \Gamma_{T}\left(\varphi(h) \otimes F_{1} \otimes \ldots \otimes F_{n}\right)=T^{c}\left(\varphi(h) \otimes F_{1} \otimes \ldots \otimes F_{n}\right)-\sum_{P} T_{\text {tree }}^{c}\left(\varphi(h) \otimes \bigotimes_{J \in P} \Gamma_{T}\left(F_{J}\right)\right) \\
& =\hbar \int d x d y h(x) \Delta^{F}(x-y) \frac{\delta}{\delta \varphi(y)}\left(T^{c}\left(F_{1} \otimes \ldots \otimes F_{n}\right)-\sum_{P} T_{\text {tree }}^{c}\left(\bigotimes_{J \in P} \Gamma_{T}\left(F_{J}\right)\right)\right)=0,
\end{aligned}
$$

where $F_{J} \equiv \otimes_{j \in J} F_{j}$ and $P$ runs through all partitions of $\{1, \ldots, n\}$.

Analogously to the conventions for $R$ - and $T$-products we sometimes write $\int d x g(x) \Gamma_{T}\left(A(x) \otimes F_{2} \ldots\right)$ for $\Gamma_{T}\left(\int d x g(x) A(x) \otimes F_{2} \ldots\right)$. Since $\Gamma_{T}$ depends only on the functionals, it fulfills the AWI: $\partial_{x}^{\mu} \Gamma_{T}\left(A(x) \otimes F_{2} \ldots\right)=\Gamma_{T}\left(\partial^{\mu} A(x) \otimes F_{2} \ldots\right)$.

In the proper vertex formalism a finite renormalization $T \rightarrow \hat{T}$ of the $T$-products is reflected in a finite renormalization $\Gamma_{T} \rightarrow \hat{\Gamma}_{T}$ of the corresponding vertex functions. To derive this we insert $\hat{T}\left(e_{\otimes}^{i S / \hbar}\right)=T_{\text {tree }}\left(e_{\otimes}^{i \hat{\Gamma}_{T}\left(e_{\otimes}^{S}\right) / \hbar}\right)$ and (4.19) into (3.28) and obtain

$$
T_{\text {tree }}\left(e_{\otimes}^{i \hat{\Gamma}_{T}\left(e_{\otimes}^{S}\right) / \hbar}\right)=T_{\text {tree }}\left(e_{\otimes}^{i \Gamma_{T}\left(e_{\otimes}^{D\left(e_{\otimes}^{S}\right)}\right) / \hbar}\right) .
$$

(Note that the tree part $T_{\text {tree }}$ is independent of the normalization of the $T$-product.) By using (4.14) we conclude

$$
\hat{\Gamma}_{T}\left(e_{\otimes}^{S}\right)=\Gamma_{T}\left(e_{\otimes}^{D\left(e_{\otimes}^{S}\right)}\right)
$$

\subsection{Comparison with the literature}

Definition of the 'vertex functional' (or 'proper function') $\Gamma$ in the literature, see e.g. [10]. Usually $\Gamma(h), h \in \mathcal{S}(\mathbb{M}, \mathbb{R})$, is defined as the Legendre transformed $j \rightarrow h$ of the generating functional $Z(j)$ of the connected Green's functions (where $j$ is the 'classical source' of $\varphi$ ). With that $\Gamma(h)$ is the generating functional of the 1PI-diagrams of $T\left(e_{\otimes}^{i S}\right)$ (see [28]). We are going to express the latter fact in our formalism. To simplify the notations we study a scalar field $\varphi$ with free action 
$S_{0}(\varphi)=1 / 2 \int d x\left((\partial \varphi(x))^{2}-m^{2}(\varphi(x))^{2}\right)$. Green's functions are obtained by the GellMann Low formula [29], which contains the adiabatic limit $g \rightarrow 1$ :

$$
G\left(x_{1}, \ldots, x_{n}\right)=\lim _{g \rightarrow 1} \frac{\omega_{0}\left(T\left(\varphi\left(x_{1}\right) \ldots \varphi\left(x_{n}\right) e^{i S(g) / \hbar}\right)\right)}{\omega_{0}\left(T\left(e^{i S(g) / \hbar}\right)\right)},
$$

where $S(g)=\sum_{n=1}^{\infty} \kappa^{n} \int d x(g(x))^{n} \mathcal{L}_{n}(x)$ and $\kappa$ is the coupling constant. All diagrams with vacuum-subdiagrams are divided out. These diagrams are disconnected and, hence, not of interest for our purposes. Namely, to obtain the vertex functional $\Gamma$ one selects all diagrams of $G\left(x_{1}, \ldots, x_{n}\right)$ which are 1PI after amputation of the external legs. The contribution of these diagrams is given by

$$
\begin{gathered}
G^{\mathrm{PI}}\left(x_{1}, \ldots, x_{n}\right)=\lim _{g \rightarrow 1} \int d y_{1} \ldots d y_{n} \Delta_{F}\left(x_{1}-y_{1}\right) \ldots \Delta_{F}\left(x_{n}-y_{n}\right) . \\
\cdot \omega_{0}\left(\frac{\delta^{n}}{\delta \varphi\left(y_{1}\right) \ldots \delta \varphi\left(y_{n}\right)} T^{1 \mathrm{PI}}\left(e^{i S(g) / \hbar}\right)\right)+\delta_{n, 2} \Delta^{F}\left(x_{1}-x_{2}\right) .
\end{gathered}
$$

¿From this expression $\Gamma(h)$ is obtained by replacing each external leg $\Delta_{F}\left(x_{l}-y_{l}\right)$ by the classical field $h\left(y_{l}\right)$. In addition one multiplies with $(-i) / n$ ! and sums over $n \geq 1:^{11}$

$$
\begin{aligned}
\Gamma(h)= & S_{0}(h)+\frac{\hbar}{i} \lim _{g \rightarrow 1} \sum_{n \geq 1} \frac{1}{n !} \int d y_{1} \ldots d y_{n} h\left(y_{1}\right) \ldots h\left(y_{n}\right) . \\
& \cdot \omega_{0}\left(\frac{\delta^{n}}{\delta \varphi\left(y_{1}\right) \ldots \delta \varphi\left(y_{n}\right)} T^{1 \mathrm{PI}}\left(e^{i S(g) / \hbar}\right)\right) .
\end{aligned}
$$

Note that the term $S_{0}(h)$ comes from the $\delta_{n, 2} \Delta^{F}$-term in (4.35).

Relation to our proper interaction $\Gamma_{T}\left(e_{\otimes}^{S}(g)\right)$ : We compare (4.36) with the Taylor expansion in $\varphi$ of $\Gamma_{T}\left(e_{\otimes}^{S(g)}\right)$ :

$$
\Gamma_{T}\left(e_{\otimes}^{S(g)}\right)=\sum_{n \geq 0} \frac{1}{n !} \int d y_{1} \ldots d y_{n} \varphi\left(y_{1}\right) \ldots \varphi\left(y_{n}\right) \omega_{0}\left(\frac{\delta^{n}}{\delta \varphi\left(y_{1}\right) \ldots \delta \varphi\left(y_{n}\right)} \Gamma_{T}\left(e_{\otimes}^{S(g)}\right)\right)
$$

and use Corollary 5. This yields

$$
\Gamma(h)=S_{0}(h)+\lim _{g \rightarrow 1}\left(\Gamma_{T}\left(e_{\otimes}^{S(g)}\right)(h)-\omega_{0}\left(\Gamma_{T}\left(e_{\otimes}^{S(g)}\right)\right)\right) .
$$

(On the right-hand side the functionals $S_{0}, \Gamma_{T}\left(e_{\otimes}^{S(g)}\right) \in \mathcal{F}$ are evaluated on the classical field configuration $h \in \mathcal{S}(\mathbb{M}, \mathbb{R})$.)

\section{The Master Ward Identity}

\subsection{The classical MWI in the off-shell formalism}

In [1] the MWI for on-shell fields (i.e. the retarded products are restricted to the solutions of the free field equation(s)) was derived in the framework of classical field

\footnotetext{
${ }^{11}$ Usually it is assumed that $\lim _{g \rightarrow 1} \omega_{0}\left(\varphi_{g} \mathcal{L}(x)\right)=0$, which implies $\left.\frac{\delta \Gamma(h)}{\delta h(x)}\right|_{h=0}=0$, i.e. the sum runs only over $n \geq 2$.
} 
theory. Since here, we work throughout in a general off-shell formalism [16], we shall derive an off-shell version of the classical MWI. In addition, we give an equivalent formulation of the classical MWI in terms of $T_{\text {tree-products that will be useful for the }}$ proper field formulation of the MWI in Sect. 5.3.

The classical off-shell MWI follows from the factorization (2.16) and the definition of the retarded wave operators. Let $\mathcal{J}$ be the ideal generated by the free field equation(s),

$$
\mathcal{J} \stackrel{\text { def }}{=}\left\{\sum_{n=1}^{N} \int d x_{1} \ldots d x_{n} \varphi\left(x_{1}\right) \cdots \varphi\left(x_{n-1}\right) \frac{\delta S_{0}}{\delta \varphi\left(x_{n}\right)} f_{n}\left(x_{1}, \ldots, x_{n}\right)\right\} \subset \mathcal{F},
$$

with $N<\infty$ and the $f_{n}$ 's being defined as in (2.2). Every $A \in \mathcal{J}$ can be written as

$$
A \stackrel{\text { def }}{=} \int d x Q(x) \frac{\delta S_{0}}{\delta \varphi(x)},
$$

where $Q$ is of the form

$$
Q(x)=\sum_{n=0}^{N} \int d x_{1} \ldots d x_{n} \varphi\left(x_{1}\right) \cdots \varphi\left(x_{n}\right) f_{n+1}\left(x_{1}, \ldots, x_{n}, x\right) .
$$

Note that in the present framework of classical field theory $Q$ does not need to be a local functional. Given $A \in \mathcal{J}$ we introduce a corresponding derivation [1]

$$
\delta_{A} \stackrel{\text { def }}{=} \int d x Q(x) \frac{\delta}{\delta \varphi(x)} .
$$

¿From the defining property of the retarded wave operators Def. 1 (ii) we obtain

$$
\begin{aligned}
\left(A+\delta_{A} S\right) \circ r_{S_{0}+S, S_{0}} & =\int d x Q(x) \circ r_{S_{0}+S, S_{0}} \frac{\delta\left(S_{0}+S\right)}{\delta \varphi(x)} \circ r_{S_{0}+S, S_{0}} \\
& =\int d x Q(x) \circ r_{S_{0}+S, S_{0}} \frac{\delta S_{0}}{\delta \varphi(x)},
\end{aligned}
$$

which reads perturbatively

$$
R_{\mathrm{cl}}\left(e_{\otimes}^{S}, A+\delta_{A} S\right)=\int d x R_{\mathrm{cl}}\left(e_{\otimes}^{S}, Q(x)\right) \frac{\delta S_{0}}{\delta \varphi(x)} \in \mathcal{J} .
$$

This is the MWI written in the general off-shell formalism. Indeed, by restricting (5.5) on solutions of the free field equation, the right-hand side vanishes and we obtain the on-shell version of the MWI, as it was derived in [1]. Note that for the simplest case $Q=1$ the MWI reduces to the off-shell version of the (interacting) field equation

$$
R_{\mathrm{cl}}\left(e_{\otimes}^{S}, \frac{\delta\left(S_{0}+S\right)}{\delta \varphi(x)}\right)=\frac{\delta S_{0}}{\delta \varphi(x)},
$$

which is an alternative formulation of the axiom Field Equation in Sect. 3. 
The classical field equation (5.6) can be expressed in the time ordered formalism:

$$
T_{\text {tree }}\left(e_{\otimes}^{i S} \otimes \frac{\delta\left(S_{0}+S\right)}{\delta \varphi(x)}\right)=\frac{\delta S_{0}}{\delta \varphi(x)} \cdot T_{\text {tree }}\left(e_{\otimes}^{i S}\right) .
$$

This identity holds even for non-local entries and can be obtained easily by using the definition of $T_{\text {tree }}$ given in (4.10) and the fact that $\Delta^{F}$ is a Green's function of the Klein Gordon operator. Similarly to $R_{\mathrm{cl}}$, the tree diagrams of the time ordered product factorize (cf. [30]), that is

$$
T_{\text {tree }}\left(e_{\otimes}^{i S} \otimes F\right) \cdot T_{\text {tree }}\left(e_{\otimes}^{i S} \otimes G\right)=T_{\text {tree }}\left(e_{\otimes}^{i S} \otimes F G\right) \cdot T_{\text {tree }}\left(e_{\otimes}^{i S}\right) .
$$

We now multiply the field equation for $T_{\text {tree }}$ with $T_{\text {tree }}\left(e_{\otimes}^{i S} \otimes Q(x)\right)$. This yields the MWI in the time ordered formalism:

$$
T_{\text {tree }}\left(e_{\otimes}^{i S} \otimes\left(A+\delta_{A} S\right)\right)=\int d x T_{\text {tree }}\left(e_{\otimes}^{i S} \otimes Q(x)\right) \cdot \frac{\delta S_{0}}{\delta \varphi(x)} .
$$

We point out that the MWI for $T_{\text {tree }}(5.9)$ holds also for non-local entries $S, Q(x)$ and $A$.

\subsection{Structure of possible anomalies of the MWI in QFT}

The classical MWI was derived for arbitrary interaction $S \in \mathcal{F}$ and arbitrary $A \in \mathcal{J}$. For local functionals $A \in \mathcal{J}_{\text {loc }} \equiv \mathcal{J} \cap \mathcal{F}_{\text {loc }}$ and $S \in \mathcal{F}_{\text {loc }}$ it can be transferred formally into pQFT (by the replacement $R_{\mathrm{cl}} \rightarrow R$ ), where it serves as an additional, highly non-trivial renormalization condition. It is impossible to fulfill this condition for all $A \in \mathcal{J}_{\text {loc }}$. We aim to find a general expression for the possible violations ('anomalies') of the MWI. Later we will use this result as starting point for a proof of relevant cases of the MWI. This procedure is motivated by algebraic renormalization, where the QAP serves as the crucial input to study the possibility to fulfill some Ward identities (see [10] and references cited therein).

The main insight into the structure of possible anomalies of the MWI is the fact that they can be expressed in terms of a local interacting field:

Theorem 7. Given a retarded product $R$ fulfilling the basic axioms Initial Condition, Causality and GLZ Relation and given a local functional

$$
A=\int d x h(x) Q(x) \frac{\delta S_{0}}{\delta \varphi(x)} \in \mathcal{J}_{\mathrm{loc}}, \quad h \in \mathcal{D}(\mathbb{M}), \quad Q \in \mathcal{P}
$$

there exists a unique, linear and symmetric map

$$
\begin{aligned}
\Delta_{A}: \mathbb{T} \mathcal{F}_{\text {loc }} & \longrightarrow \mathcal{F}_{\text {loc }} \\
F_{1} \otimes \cdots \otimes F_{n} & \longmapsto \Delta_{A}\left(F_{1} \otimes \cdots \otimes F_{n}\right)
\end{aligned}
$$

which is implicitly defined by the 'anomalous MWI'

$$
R\left(e_{\otimes}^{S}, A+\delta_{A} S+\Delta_{A}\left(e_{\otimes}^{S}\right)\right)=\int d y h(y) R\left(e_{\otimes}^{S}, Q(y)\right) \frac{\delta S_{0}}{\delta \varphi(y)} .
$$


As a consequence of (5.12) the map $\Delta_{A}$ has the following properties:

(i) $\quad \Delta_{A}$ depends linearly on $A$;

(ii) locality expressed by the two relations:

(iia) $\omega_{0}\left(\Delta_{A}\left(\otimes_{j=1}^{n} F_{j}\right)\right)=0$ if $\cap_{i=1}^{n} \operatorname{supp}\left(\frac{\delta F_{i}}{\delta \varphi}\right) \cap \operatorname{supp}\left(\frac{\delta A}{\delta \varphi}\right)=\emptyset$,

(iib) $\operatorname{supp}\left(\frac{\delta \Delta_{A}\left(\otimes_{j=1}^{n} F_{j}\right)}{\delta \varphi}\right) \subset \cap_{i=1}^{n} \operatorname{supp}\left(\frac{\delta F_{i}}{\delta \varphi}\right) \cap \operatorname{supp}\left(\frac{\delta A}{\delta \varphi}\right)$;

(iii) $\Delta_{A}(1)=0$;

(iv) $\Delta_{A} \equiv 0 \Leftrightarrow R\left(e_{\otimes}^{S}, A+\delta_{A} S\right)=\int d x h(x) R\left(e_{\otimes}^{S}, Q(x)\right) \frac{\delta S_{0}}{\delta \varphi(x)}, \forall S \in \mathcal{F}_{\text {loc }} ;$

(v) $\quad \Delta_{A}\left(F_{1} \otimes \cdots \otimes F_{n}\right)=\mathcal{O}(\hbar) \quad \forall n>0, F_{i} \sim \hbar^{0}$,

and

(vi) We set $\left.\Delta_{A}^{n} \equiv \Delta_{A}\right|_{\mathcal{F}_{\text {loc }}^{\otimes n}}$. For $g_{j} \in \mathcal{D}(\mathbb{M}), L_{j} \in \mathcal{P}$ it holds

$$
\begin{gathered}
\Delta_{A}^{n}\left(L_{1}\left(g_{1}\right) \otimes \ldots \otimes L_{n}\left(g_{n}\right)\right)=\int d x_{1} \ldots d x_{n} d y g_{1}\left(x_{1}\right) \ldots g_{n}\left(x_{n}\right) h(y) \\
\cdot \Delta^{n}\left(L_{1}\left(x_{1}\right) \otimes \ldots \otimes L_{n}\left(x_{n}\right) ; Q(y)\right),
\end{gathered}
$$

where the distributional kernel $\Delta^{n}\left(L_{1}\left(x_{1}\right) \otimes \ldots ; Q(y)\right)$ is inductively given by

$$
\begin{gathered}
\Delta^{n}\left(L_{1}\left(x_{1}\right) \otimes \ldots \otimes L_{n}\left(x_{n}\right) ; Q(y)\right)=-R\left(\otimes_{j=1}^{n} L_{j}\left(x_{j}\right) ; Q(y) \cdot \frac{\delta S_{0}}{\delta \varphi(y)}\right) \\
-\sum_{l=1}^{n} R\left(\otimes_{j(\neq l)} L_{j}\left(x_{j}\right) ; Q(y) \sum_{a}\left(\partial^{a} \delta\right)\left(x_{l}-y\right) \frac{\partial L_{l}}{\partial\left(\partial^{a} \varphi\right)}\left(x_{l}\right)\right) \\
-\sum_{I \subset\{1, \ldots, n\}, I \neq \emptyset} R\left(\otimes_{i \in I} L_{i}\left(x_{i}\right) ; \Delta^{\left|I^{c}\right|}\left(\otimes_{j \in I^{c}} L_{j}\left(x_{j}\right) ; Q(y)\right)\right) \\
+R\left(\otimes_{j=1}^{n} L_{j}\left(x_{j}\right) ; Q(y)\right) \cdot \frac{\delta S_{0}}{\delta \varphi(y)} .
\end{gathered}
$$

Note that (5.12) differs from the MWI (5.5) only by the local term $\Delta_{A}\left(e_{\otimes}^{S}\right)$, which clearly depends on the chosen normalization of the retarded products. Therefore, property $(i v)$ means that the MWI for $A$ is fulfilled if and only if the corresponding map $\Delta_{A}$ vanishes identically.

Proof. To show the existence and uniqueness of $\Delta_{A}$ we construct its components $\Delta_{A}^{n}$ by induction on $n$ using (5.12). In this inductive procedure we also prove the properties $(i)-(i i i)$ and $(v i)$. To lowest order in $S$ the condition $(5.12)$ gives $\Delta_{A}(1) \stackrel{\text { def }}{=} 0$. Given $n>0$, we assume the existence and uniqueness of linear and symmetrical maps $\Delta_{A}^{k}: \mathcal{F}_{\text {loc }}^{\otimes k} \rightarrow \mathcal{F}_{\text {loc }}, 0<k<n$, which depend linearly on $A$, are local and satisfy (vi), 
such that (5.12) is fulfilled to all lower orders in $S$ :

$$
\begin{gathered}
R\left(S^{\otimes k}, A\right)+k R\left(S^{\otimes k-1}, \delta_{A} S\right)+\sum_{l=0}^{k}\left(\begin{array}{l}
k \\
l
\end{array}\right) R\left(S^{\otimes k-l}, \Delta_{A}^{l}\left(S^{\otimes l}\right)\right)= \\
\int d x h(x) R\left(S^{\otimes k}, Q(x)\right) \frac{\delta S_{0}}{\delta \varphi(x)}
\end{gathered}
$$

for all $k<n$. We define $\Delta_{A}^{n}$ in terms of the inductively known $\Delta_{A}^{k}, k<n$ :

$$
\begin{aligned}
\Delta_{A}^{n}\left(F_{1} \otimes \cdots \otimes F_{n}\right) \stackrel{\text { def }}{=} & \int d x h(x) R\left(F_{1} \otimes \cdots \otimes F_{n}, Q(x)\right) \frac{\delta S_{0}}{\delta \varphi(x)} \\
- & \left(R\left(F_{1} \otimes \cdots \otimes F_{n}, A\right)+\sum_{k=1}^{n} R\left(\otimes_{i \in \underline{n} \backslash\{k\}} F_{i}, \delta_{A} F_{k}\right)\right. \\
& \left.+\sum_{I \sqcup J=\underline{n},|J|<n} R\left(\otimes_{i \in I} F_{i}, \Delta_{A}^{|J|}\left(\otimes_{j \in J} F_{j}\right)\right)\right),
\end{aligned}
$$

where we used the notation $\underline{n} \stackrel{\text { def }}{=}\{1, \ldots, n\}$ and $I \sqcup J$ for the disjoint union of $I$ and $J$. For $F_{1}=\ldots=F_{n}=S$ the formula (5.17) agrees with (5.12) to order $n$ in $S$. Obviously, $\Delta_{A}^{n}$ is linear and symmetrical and it is uniquely determined by these properties and (5.12). We also see that $\Delta_{A}^{n}$ is linear in $Q$ resp. $A$.

The main task is to show the locality (ii) of the right-hand side of (5.17) (which also implies $\left.\Delta_{A}^{n}\left(F_{1} \otimes \cdots \otimes F_{n}\right) \in \mathcal{F}_{\text {loc }}\right)$. To this end we denote by

$$
\begin{aligned}
& M_{n, 1}\left(L_{1}\left(x_{1}\right), \ldots, L_{n}\left(x_{n}\right) ; Q(y)\right) \stackrel{\stackrel{\text { def }}{=}}{=} R\left(\otimes_{i \in \underline{n}} L_{i}\left(x_{i}\right), Q(y) \frac{\delta S_{0}}{\delta \varphi(y)}\right) \\
&+\sum_{k=1}^{n} R\left(\otimes_{i \in \underline{n} \backslash\{k\}} L_{i}\left(x_{i}\right), Q(y) \frac{\delta L_{k}\left(x_{k}\right)}{\delta \varphi(y)}\right) \\
&+\sum_{I \sqcup J=\underline{n},|J|<n} R\left(\otimes_{i \in I} L_{i}\left(x_{i}\right), \Delta^{|J|}\left(\otimes_{j \in J} L_{j}\left(x_{j}\right) ; Q(y)\right)\right)
\end{aligned}
$$

the distributional kernel of the three terms in brackets in equation (5.17), where $F_{i}=$ $\int d x g_{i}(x) L_{i}(x)\left(g_{i} \in \mathcal{D}(\mathbb{M}), L_{i} \in \mathcal{P}\right)$ and we use (5.14) for $\Delta_{A}^{|J|}$ to lower orders $|J|<n$. We will show that $M_{n, 1}\left(L_{1}\left(x_{1}\right), \ldots ; Q(y)\right)$ coincides outside the total diagonal $\mathbb{D}_{n+1}=\left\{\left(x_{1}, \ldots, x_{n+1}\right) \in \mathbb{M}^{n+1}, x_{1}=\cdots=x_{n+1}\right\}$ with the distributional kernel $R\left(L_{1}\left(x_{1}\right) \otimes \cdots \otimes L_{n}\left(x_{n}\right), Q(y)\right) \frac{\delta S_{0}}{\delta \varphi(y)}$ of the first term in (5.17). This shows

$$
\operatorname{supp} \Delta^{n}\left(\otimes_{j=1}^{n} L_{j} ; Q\right) \subset \mathbb{D}_{n+1},
$$

where $\Delta^{n}\left(\otimes_{j=1}^{n} L_{j} ; Q\right)$ is defined by (5.15). (By construction $\Delta^{n}\left(\otimes_{j=1}^{n} L_{j} ; Q\right)$ is the distributional kernel of $\Delta_{A}^{n}\left(L_{1}\left(g_{1}\right) \otimes \ldots\right)(5.17)$, which proves (vi).) The support property (5.18) implies locality (iia). To derive the the second locality statement (iib) we additionally use that

$$
\frac{\delta R\left(\otimes_{j=1}^{k-1} A_{j}\left(x_{j}\right) ; A_{k}\left(x_{k}\right)\right)}{\delta \varphi(z)}=0 \quad \text { if } \quad z \neq x_{j} \forall j=1, \ldots, k,
$$


as explained in [30]. By means of (5.15) we conclude

$$
\operatorname{supp} \frac{\delta \Delta^{n}\left(\otimes_{j=1}^{n} L_{j} ; Q\right)}{\delta \varphi} \subset \mathbb{D}_{n+2},
$$

which is equivalent to locality $(i i b)$. It follows also $\Delta_{A}^{n}\left(\otimes_{j=1}^{n} L_{j}\left(g_{j}\right)\right) \in \mathcal{F}_{\text {loc }}$.

We turn to the proof of (5.18). Since $M_{n, 1}$ has retarded support

$$
\operatorname{supp}\left(\delta M_{n, 1} / \delta \varphi\right) \subset\left\{\left(x_{1}, \ldots, x_{n}, y\right) \in \mathbb{M}^{n+1} ; x_{1}, \ldots, x_{n} \in y+\bar{V}_{-}\right\}
$$

and is symmetrical under permutation of the first $n$ entries, it is thereby sufficient to consider the particular case where $x_{n} \in y+\bar{V}_{-} \backslash\{y\}$. In this case it holds

$$
\begin{aligned}
M_{n, 1}\left(L_{1}\left(x_{1}\right), \ldots ; Q(y)\right) & =R\left(\otimes_{i \in \underline{n-1}} L_{i}\left(x_{i}\right) \otimes L_{n}\left(x_{n}\right), Q(y) \frac{\delta S_{0}}{\delta \varphi(y)}\right) \\
& +\sum_{k=1}^{n-1} R\left(\otimes_{i \in \underline{n} \backslash\{k\}} L_{i}\left(x_{i}\right) \otimes L_{n}\left(x_{n}\right), Q(y) \frac{\delta L_{k}\left(x_{k}\right)}{\delta \varphi(y)}\right) \\
& +\sum_{I \sqcup J=\underline{n-1}} R\left(\otimes_{i \in I} L_{i}\left(x_{i}\right) \otimes L_{n}\left(x_{n}\right), \Delta^{|J|}\left(\otimes_{j \in J} L_{j}\left(x_{j}\right) ; Q(y)\right)\right)
\end{aligned}
$$

where the locality of the distributions $\Delta^{|J|}\left(\otimes_{j \in J} L_{j}\left(x_{j}\right) ; Q(y)\right)$ for $|J|<n$ is used. Now we apply the GLZ Relation to each term and take the support property of the retarded products into account. This yields

$$
\begin{aligned}
& M_{n, 1}\left(L\left(x_{1}\right), \ldots ; Q(y)\right)=\sum_{I \sqcup J=\underline{n-1}}\left\{R\left(\otimes_{i \in I} L_{i}\left(x_{i}\right), L_{n}\left(x_{n}\right)\right), R\left(\otimes_{j \in J} L_{j}\left(x_{j}\right), Q(y) \frac{\delta S_{0}}{\delta \varphi(y)}\right)\right\} \\
& \quad+\sum_{k=1}^{n-1} \sum_{I \sqcup J \sqcup\{k\}=\underline{n-1}}\left\{R\left(\otimes_{i \in I} L_{i}\left(x_{i}\right), L_{n}\left(x_{n}\right)\right), R\left(\otimes_{j \in J} L_{j}\left(x_{j}\right), Q(y) \frac{\delta L_{k}\left(x_{k}\right)}{\delta \varphi(y)}\right)\right\} \\
& +\sum_{I \sqcup J=\underline{n-1}} \sum_{K \sqcup L=I}\left\{R\left(\otimes_{i \in K} L_{i}\left(x_{i}\right), L_{n}\left(x_{n}\right)\right), R\left(\otimes_{l \in L} L_{l}\left(x_{l}\right), \Delta^{|J|}\left(\otimes_{j \in J} L_{j}\left(x_{j}\right) ; Q(y)\right)\right)\right\} .
\end{aligned}
$$

To transform the last term we use the induction hypothesis (5.16) written in distributional form,

$$
\begin{gathered}
\sum_{L \sqcup J=H} R\left(\otimes_{l \in L} L_{l}\left(x_{l}\right), \Delta^{|J|}\left(\otimes_{j \in J} L_{j}\left(x_{j}\right) ; Q(y)\right)\right)=R\left(\otimes_{i \in H} L_{i}\left(x_{i}\right), Q(y)\right) \frac{\delta S_{0}}{\delta \varphi(y)} \\
-R\left(\otimes_{i \in H} L_{i}\left(x_{i}\right), Q(y) \frac{\delta S_{0}}{\delta \varphi(y)}\right)-\sum_{j \in H} R\left(\otimes_{i \in H \backslash\{j\}} L_{i}\left(x_{i}\right), Q(y) \frac{\delta L_{j}\left(x_{j}\right)}{\delta \varphi(y)}\right)
\end{gathered}
$$


for all $H \subset \underline{n-1}$. After rearranging the sums the last term in (5.20) is equal to

$$
\begin{aligned}
& -\sum_{I \sqcup J=\underline{n-1}}\left\{R\left(\otimes_{i \in I} L_{i}\left(x_{i}\right), L_{n}\left(x_{n}\right)\right), R\left(\otimes_{j \in J} L_{j}\left(x_{j}\right), Q(y) \frac{\delta S_{0}}{\delta \varphi(y)}\right)\right\} \\
- & \sum_{k=1}^{n-1} \sum_{I \sqcup J \sqcup\{k\}=\underline{n-1}}\left\{R\left(\otimes_{i \in I} L_{i}\left(x_{i}\right), L_{n}\left(x_{n}\right)\right), R\left(\otimes_{j \in J} L_{j}\left(x_{j}\right), Q(y) \frac{\delta L_{k}\left(x_{k}\right)}{\delta \varphi(y)}\right)\right\} \\
& +\sum_{I \sqcup J=\underline{n-1}}\left\{R\left(\otimes_{i \in I} L_{i}\left(x_{i}\right), L_{n}\left(x_{n}\right)\right), R\left(\otimes_{j \in J} L_{j}\left(x_{j}\right), Q(y)\right) \frac{\delta S_{0}}{\delta \varphi(y)}\right\} .
\end{aligned}
$$

The last term in (5.22) can be transformed by using the identity

$$
\left\{F, P(x) \frac{\delta S_{0}}{\delta \varphi(x)}\right\}=\{F, P(x)\} \frac{\delta S_{0}}{\delta \varphi(x)}, \quad F, P(x) \in \mathcal{F}
$$

(which follows from the fact that the 2-point function $H_{m}$ is a solution of the free field equation) and by applying the GLZ Relation:

$$
\begin{aligned}
\sum_{I \sqcup J=\underline{n-1}}\{ & \left.R\left(\otimes_{i \in I} L_{i}\left(x_{i}\right), L_{n}\left(x_{n}\right)\right), R\left(\otimes_{j \in J} L_{j}\left(x_{j}\right), Q(y)\right) \frac{\delta S_{0}}{\delta \varphi(y)}\right\} \\
& =\sum_{I \sqcup J=\underline{n-1}}\left\{R\left(\otimes_{i \in I} L_{i}\left(x_{i}\right), L_{n}\left(x_{n}\right)\right), R\left(\otimes_{j \in J} L_{j}\left(x_{j}\right), Q(y)\right)\right\} \frac{\delta S_{0}}{\delta \varphi(y)} \\
& =R\left(L_{1}\left(x_{1}\right) \otimes \cdots \otimes L_{n}\left(x_{n}\right), Q(y)\right) \frac{\delta S_{0}}{\delta \varphi(y)} .
\end{aligned}
$$

Summing up the first two terms in (5.20) and the first two terms in (5.22) cancel and we get the desired result

$$
M_{n, 1}\left(L_{1}\left(x_{1}\right), \ldots Q(y)\right)=R\left(L_{1}\left(x_{1}\right) \otimes \cdots \otimes L_{n}\left(x_{n}\right), Q(y)\right) \frac{\delta S_{0}}{\delta \varphi(y)}
$$

$\forall\left(x_{1}, \ldots, x_{n}, y\right) \notin \mathbb{D}_{n+1}$ which proves (5.18).

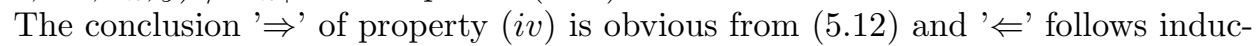
tively for $\Delta_{A}^{n}\left(S^{\otimes n}\right)$, because the right-hand side of (5.17) (for $F_{1}=\ldots=F_{n}=S$ ) vanishes in that case if $\Delta_{A}^{k} \equiv 0 \quad \forall k<n$. And $\Delta_{A}\left(e_{\otimes}^{S}\right)=0 \forall S$ implies $\Delta_{A} \equiv 0$ by the polarization identity.

The crucial property that $\Delta_{A}\left(F_{1} \otimes \cdots \otimes F_{n}\right)=\mathcal{O}(\hbar)$ for all $n>0$ and $F_{i} \sim \hbar^{0}$, follows immediately from the validity of the classical MWI and $\lim _{\hbar \rightarrow 0} R=R_{\mathrm{cl}}$ by using property $(i v)$.

Mostly we will omit the index $n$ of $\Delta_{A}^{n}$ and its kernel $\Delta^{n}$.

Up to here we only assumed that the $R$-product satisfies the basic axioms. If it fulfills renormalization conditions, then corresponding properties of $\Delta_{A}$ are implied.

Lemma 8. (i) The axioms $\mathcal{P}_{+}^{\uparrow}$-Covariance, Unitarity, Field Independence and Field Equation, respectively, imply corresponding properties of $\Delta_{A}$ :

- $\mathcal{P}_{+}^{\uparrow}$-Covariance $\beta_{L} \Delta_{A}\left(e_{\otimes}^{S}\right)=\Delta_{\beta_{L} A}\left(e_{\otimes}^{\beta_{L} S}\right), \forall L \in \mathcal{P}_{+}^{\uparrow} ;$ 
- Unitarity $\Delta_{A}\left(e_{\otimes}^{S}\right)^{\star}=\Delta_{A^{\star}}\left(e_{\otimes}^{S^{\star}}\right) ;$

- Field Independence

$$
\frac{\delta}{\delta \varphi(x)} \Delta_{A}\left(S^{\otimes n}\right)=\Delta_{A_{x}}\left(S^{\otimes n}\right)+n \Delta_{A}\left(S^{\otimes(n-1)} \otimes \frac{\delta S}{\delta \varphi(x)}\right),
$$

where $A_{x}$ is obtained from $A$ (5.10) by replacing $Q(y)$ by $\frac{\delta Q(y)}{\delta \varphi(x)}$ :

$$
A_{x} \stackrel{\text { def }}{=} \int d y h(y) \frac{\delta Q(y)}{\delta \varphi(x)} \frac{\delta S_{0}}{\delta \varphi(y)} \in \mathcal{J}_{S_{0}}
$$

\section{- Field Equation}

$$
\Delta_{A} \equiv 0 \quad \text { if } \quad A=\int d x h(x) \frac{\delta S_{0}}{\delta \varphi(x)}, \quad \forall h \in \mathcal{D}(\mathbb{M})
$$

(ii) Let $g_{j} \in \mathcal{D}(\mathbb{M}), L_{j} \in \mathcal{P}$. Assuming that the $R$-products satisfy the axioms Translation Invariance and Field Independence, there exist linear maps $P_{a}^{n}$ : $\mathcal{P}^{\otimes(n+1)} \rightarrow \mathcal{P}$ (where a runs through a finite subset of $\left.\left(\mathbb{N}_{0}^{d}\right)^{n}\right)$, which are symmetric in the first $n$ factors, such that $\Delta_{A}^{n}$ can be written as

$$
\begin{gathered}
\Delta_{A}^{n}\left(L_{1}\left(g_{1}\right) \otimes \ldots \otimes L_{n}\left(g_{n}\right)\right)= \\
\sum_{a \in\left(\mathbb{N}_{0}^{d}\right)^{n}} \int d x h(x) \partial^{a_{1}} g_{1}(x) \ldots \partial^{a_{n}} g_{n}(x) P_{a}^{n}\left(L_{1} \otimes \ldots \otimes L_{n} ; Q\right)(x) .
\end{gathered}
$$

Part (ii) can be proved without assuming Field Independence of the $R$-products, see [30].

Proof. (i) We prove all properties for the components $\Delta_{A}^{n}$ of $\Delta_{A}$ by induction on $n$. We verify that the right-hand side of (5.17) fulfills the assertion by using the pertinent property of the $R$-product and the inductive assumption.

In case of the Field Independence we additionally take

$$
\begin{gathered}
\frac{\delta A}{\delta \varphi(x)}=A_{x}+\int d y h(y) Q(y) \frac{\delta^{2} S_{0}}{\delta \varphi(y) \delta \varphi(x)}, \\
\frac{\delta\left(\delta_{A} S\right)}{\delta \varphi(x)}=\delta_{A_{x}} S+\delta_{A}\left(\frac{\delta S}{\delta \varphi(x)}\right)
\end{gathered}
$$

into account.

Field Equation: for $A=\int d x h(x) \frac{\delta S_{0}}{\delta \varphi(x)}$ the MWI reduces to the off-shell field equation in differential form, which is equivalent to the integrated version (3.8). The assertion follows by means of property $(i v)$ of $\Delta_{A}$ in Theorem 7 .

(ii) The statement is a simplified version of Proposition 4.3 in [16]. The proof, which is given there in words, is carried out here explicitly, since these formulas will be used below in the proof of part (ii) of Prop. 10 . 
The Field Independence (5.25) translates into a corresponding relation for the distributional kernel (5.14):

$$
\begin{gathered}
\frac{\delta}{\delta \varphi(x)} \Delta\left(\otimes_{j=1}^{n} L_{j}\left(x_{j}\right) ; Q(y)\right)= \\
\sum_{l=1}^{n} \Delta\left(\otimes_{j(\neq l)} L_{j}\left(x_{j}\right) \otimes \frac{\delta L_{l}\left(x_{l}\right)}{\delta \varphi(x)} ; Q(y)\right)+\Delta\left(\otimes_{j=1}^{n} L_{j}\left(x_{j}\right) ; \frac{\delta Q(y)}{\delta \varphi(x)}\right) .
\end{gathered}
$$

We insert this identity into the Taylor expansion of $\Delta\left(\otimes_{j=1}^{n} L_{j}\left(x_{j}\right) ; Q(y)\right)$ with respect to $\varphi$. This yields the causal Wick expansion

$$
\begin{gathered}
\Delta\left(\otimes_{j=1}^{n} L_{j}\left(x_{j}\right) ; Q(y)\right)=\sum_{l_{1}, \ldots ; l} \sum_{a_{i j_{i}}, a_{j}} C_{a_{11} \ldots a_{1 l_{1}}, \ldots ; a_{1} \ldots a_{l}}^{l_{1} \ldots l} \\
\cdot \omega_{0}\left(\Delta\left(\otimes_{i=1}^{n} \frac{\partial^{l_{i}} L_{i}}{\partial\left(\partial^{a_{i 1}} \varphi\right) \ldots \partial\left(\partial^{a_{i l_{i}}} \varphi\right)}\left(x_{i}\right) ; \frac{\partial^{l} Q}{\partial\left(\partial^{a_{1}} \varphi\right) \ldots \partial\left(\partial^{a_{l}} \varphi\right)}(y)\right)\right) \\
\cdot \prod_{i=1}^{n}\left(\prod_{j_{i}=1}^{l_{i}} \partial^{a_{i j_{i}}} \varphi\left(x_{i}\right)\right) \cdot \prod_{j=1}^{l} \partial^{a_{j}} \varphi(y),
\end{gathered}
$$

where $C_{a_{11} \ldots ; a_{11}, \ldots ; a_{1} \ldots a_{l}}^{l_{1} \ldots ;}$ is a combinatorial factor. Now we crucially use the locality $($ iia $)$ of $\omega_{0}\left(\Delta\left(\frac{\partial^{l_{1}} L_{1}}{\partial \ldots}\left(x_{1}\right) \otimes \ldots ; \frac{\partial^{l} Q}{\partial \ldots}(y)\right)\right)$. Hence this distribution is of the form

$$
\omega_{0}\left(\Delta\left(\frac{\partial^{l_{1}} L_{1}}{\partial \ldots}\left(x_{1}\right) \otimes \ldots ; \frac{\partial^{l} Q}{\partial \ldots}(y)\right)\right)=\sum_{b} C_{b}\left(\partial^{b} \delta\right)\left(x_{1}-y, \ldots, x_{n}-y\right),
$$

with constant numbers $C_{b}$ (due to Translation Invariance) which depend on the field polynomials in the argument of $\Delta$. The term with index $b$ gives the following contribution to $\Delta_{A}\left(L_{1}\left(g_{1}\right) \otimes \ldots \otimes L_{n}\left(g_{n}\right)\right)(5.14)$ :

$$
\begin{gathered}
(-1)^{|b|} \int d x_{1} \ldots d x_{n} d y \delta\left(x_{1}-y, \ldots, x_{n}-y\right) \\
\cdot h(y) \cdot \partial_{x_{1} \ldots x_{n}}^{b}\left(g_{1}\left(x_{1}\right) \ldots g_{n}\left(x_{n}\right) \cdot \prod_{i=1}^{n}\left(\prod_{j_{i}=1}^{l_{i}} \partial^{a_{i j_{i}}} \varphi\left(x_{i}\right)\right) \cdot \prod_{j=1}^{l} \partial^{a_{j}} \varphi(y)\right),
\end{gathered}
$$

where we have omitted constant factors. By reordering the sums we write $\Delta_{A}\left(L_{1}\left(g_{1}\right) \otimes\right.$ $\left.\ldots \otimes L_{n}\left(g_{n}\right)\right)$ in the form (5.28). Since $\Delta_{A}\left(L_{1}\left(g_{1}\right) \otimes \ldots \otimes L_{n}\left(g_{n}\right)\right)$ is multilinear in the fields $L_{1}, \ldots, L_{n}, Q$ and symmetric in $L_{1}, \ldots, L_{n}$, the maps $P_{a}^{n}$ must satisfy corresponding properties.

We turn to the scaling behaviour of $\Delta_{A}$. Assuming that the $R$-products satisfy the axioms Smoothness in $m \geq 0, \mu$-Covariance and almost homogeneous Scaling (3.16), the map $\left(S^{\otimes n} \otimes A\right) \mapsto \Delta_{A}^{(m, \mu)}\left(S^{\otimes n}\right)$ does not scale almost homogeneously in general. Namely, for non-vanishing mass we are faced with $m$-dependent inhomogeneous polynomials: $S_{0}^{(m)} \equiv S_{0} \notin \mathcal{P}_{\text {hom }}, \frac{\delta S_{0}^{(m)}}{\delta \varphi} \notin \mathcal{P}_{\text {hom }}$ and, hence, $A^{(m)} \equiv A$ is in general not 
in $\mathcal{P}_{\text {hom. }}$. In typical applications of the MWI (see e.g. the $O(N)$-model in Sect. (5.4.4) or current conservation in QED) the simplification appears that $Q_{j}$ is independent of $m$ and the $m$-dependent terms of $\frac{\delta S_{0}}{\delta \varphi_{j}}$ cancel in $A=\int d x \sum_{j} Q_{j}(x) \frac{\delta S_{0}}{\delta \varphi_{j}(x)}$. But even with that, $\sigma_{\rho} \Delta_{\sigma_{\rho}^{-1} A}^{\left(\rho^{-1} m, \mu\right)}\left(\left(\sigma_{\rho}^{-1} S\right)^{\otimes n}\right)$ is in general not a polynomial in $(\log \rho)$ : proceeding inductively the first term on the right-hand side of (5.17) does not scale almost homogeneously due to the mass term in $\frac{\delta S_{0}}{\delta \varphi} \cdot{ }^{12}$

For this reason, we assume here the axiom Scaling Degree (3.19) instead of Smoothness in $m, \mu$-Covariance and almost homogeneous Scaling.

With this weaker assumption we are going to derive a corresponding scaling degree property of $\Delta_{A}$ and an upper bound for the mass dimension of $\Delta_{A}\left(S^{\otimes n}\right)$ which does not depend on $n$ if $S$ is a renormalizable interaction. For the latter purpose we have to define the mass dimension of a local functional. We use that every $F \in \mathcal{F}_{\text {loc }}$ can uniquely be written in the form

$$
F=\sum_{i} \int d x g_{i}(x) P_{i}(x), \quad g_{i} \in \mathcal{D}(\mathbb{M}), P_{i} \in \mathcal{P}_{\text {bal }} .
$$

With that we define

$$
\operatorname{dim} F \stackrel{\text { def }}{=} \max _{i} \operatorname{dim} P_{i}
$$

This definition is minimal in the following sense:

Lemma 9. Let

$$
F=\sum_{i} \int d x g_{i}(x) B_{i}(x), \quad g_{i} \in \mathcal{D}(\mathbb{M}), B_{i} \in \mathcal{P} .
$$

Then it holds

$$
\operatorname{dim} F \leq \max _{i} \operatorname{dim} B_{i}
$$

Proof. Every $B_{i}$ can uniquely be written as $B_{i}=\sum_{j} p_{i j}(\partial) P_{i j}, P_{i j} \in \mathcal{P}_{\text {bal }}$, where $p_{i j}(\partial)$ is a polynomial in the partial derivatives [16]. Inserting this into (5.37) and shifting the derivatives to the test function we obtain the unique representation (5.35) of $F$. The assertion follows from $\operatorname{dim} P_{i j} \leq \operatorname{dim} B_{i} \forall i, j$.

¿From $A=\int d y h(y) Q(y) \frac{\delta S_{0}}{\delta \varphi(y)}$ we conclude

$$
\operatorname{dim}(A) \leq \operatorname{dim}(Q)+\operatorname{dim}\left(\frac{\delta S_{0}}{\delta \varphi}\right) \quad \text { where } \quad \operatorname{dim}\left(\frac{\delta S_{0}}{\delta \varphi}\right)=\operatorname{dim}\left(\left(\square+m^{2}\right) \varphi\right)=\frac{d+2}{2} .
$$

Analogously the relation $\delta_{A} S=\int d y h(y) Q(y) \frac{\delta S}{\delta \varphi(y)}$ implies

$$
\operatorname{dim}\left(\delta_{A} S\right) \leq \operatorname{dim}(Q)+\operatorname{dim}(S)-\operatorname{dim}(\varphi)=\operatorname{dim}(Q)+\operatorname{dim}(S)-\frac{d-2}{2} .
$$

\footnotetext{
${ }^{12}$ In detail: from the derivation of the classical MWI (5.4)-(5.5) it follows that the scaling of the right-hand side of the anomalous MWI (5.12) must be such that the mass $m$ in $R^{(m, \mu)}$ and in $\frac{\delta S_{0}^{(m)}}{\delta \varphi(y)}$ has the same value that is the scale transformation reads $\int d y h(y) \sigma_{\rho} \circ$ $R^{\left(\rho^{-1} m, \mu\right)}\left(e_{\otimes}^{\sigma_{\rho}^{-1} S}, \sigma_{\rho}^{-1} Q(y)\right) \frac{\delta S_{0}^{\left(\rho^{-1} m\right)}}{\delta \varphi(y)}$.
} 
With these tools we are ready to formulate and prove the following Proposition.

Proposition 10. (i) Scaling Degree. If the R-products fulfill the axiom Scaling Degree (3.19), then the scaling degree of the 'vacuum expectation value' of $\Delta\left(L_{1}\left(x_{1}\right) \otimes \ldots ; Q(y)\right)$ is bounded by

$$
\operatorname{sd} \omega_{0}\left(\Delta\left(\otimes_{j=1}^{n} L_{j}\left(x_{j}\right) ; Q(y)\right)\right) \leq \sum_{i=1}^{n} \operatorname{dim} L_{i}+\operatorname{dim} Q+\frac{d+2}{2} .
$$

(ii) Mass Dimension. If the R-products fulfill the axioms Field Independence and Scaling Degree (3.19), then the mass dimension of $\Delta_{A}\left(F_{1} \otimes \ldots \otimes F_{n}\right)$ is bounded by

$$
\operatorname{dim} \Delta_{A}\left(F_{1} \otimes \ldots \otimes F_{n}\right) \leq \sum_{i=1}^{n} \operatorname{dim}\left(F_{i}\right)+\operatorname{dim} Q+\frac{d+2}{2}-d n
$$

For a renormalizable interaction, that is $\operatorname{dim}(S) \leq d$, this implies

$$
\operatorname{dim} \Delta_{A}\left(e_{\otimes}^{S}\right) \leq \operatorname{dim} Q+\frac{d+2}{2} .
$$

Note that for a renormalizable interaction the upper bounds on the mass dimension of $A(5.39), \delta_{A} S(5.40)$ and $\Delta_{A}\left(e_{\otimes}^{S}\right)$ agree, as one expects because the sum $\left(A+\delta_{A} S+\right.$ $\left.\Delta_{A}\left(e_{\otimes}^{S}\right)\right)$ appears in Theorem 7 .

Proof. (i) Proceeding by induction on $n$ we apply $\omega_{0}$ to (5.15) and estimate the scaling degree of the resulting terms on the right-hand side by using

$$
\operatorname{sd} \omega_{0}\left(R\left(\otimes_{j=1}^{n-1} L_{j}\left(x_{j}\right) ; L_{n}\left(x_{n}\right)\right)\right) \leq \sum_{j=1}^{n} \operatorname{dim} L_{j}
$$

which follows from (3.19). (In contrast to (3.19) we do not assume $L_{j} \in \mathcal{P}_{\text {hom. }}$ ) For example we obtain

$$
\begin{gathered}
\operatorname{sd} \omega_{0}\left(R\left(\otimes_{j(\neq l)} L_{j}\left(x_{j}\right) ; Q(y)\left(\partial^{a} \delta\right)\left(x_{l}-y\right) \frac{\partial L_{l}}{\partial\left(\partial^{a} \varphi\right)}\left(x_{l}\right)\right)\right) \\
\leq \sum_{j(\neq l)} \operatorname{dim} L_{j}+\operatorname{dim} Q+\operatorname{dim}\left(\frac{\partial L_{l}}{\partial\left(\partial^{a} \varphi\right)}\right)+\operatorname{sd}\left(\partial^{a} \delta\right) \\
=\sum_{j=1}^{n} \operatorname{dim} L_{j}+\operatorname{dim} Q+\frac{d+2}{2} .
\end{gathered}
$$

The same bound results for all other terms. (The vacuum expectation value of the last term vanishes.)

(iv) We may assume $F_{j}=L_{j}\left(g_{j}\right)$ with $L_{j}$ a balanced field, $L_{j} \in \mathcal{P}_{\text {bal }}$. With that it holds $\operatorname{dim}\left(L_{j}\right)=\operatorname{dim}\left(F_{j}\right)$. We use the formulas derived in the proof of part $(i i)$ of Lemma 8 , in particular the causal Wick expansion of $\Delta\left(\otimes_{j} L_{j}\left(x_{j}\right) ; Q(y)\right)$ (5.32). (In 
the formulas given below the indices have precisely the same meaning as in that proof.) Looking at (5.33), we get an upper bound for the scaling degree of the left-hand side by means of (5.41). This implies that the range of $b$ on the right-hand side of (5.33) is restricted by

$$
\begin{aligned}
|b|+ & d n \leq \sum_{i=1}^{n}\left(\operatorname{dim} L_{i}-\sum_{j_{i}=1}^{l_{i}}\left(\left|a_{i j_{i}}\right|+\frac{d-2}{2}\right)\right) \\
& +\operatorname{dim} Q-\sum_{j=1}^{l}\left(\left|a_{j}\right|+\frac{d-2}{2}\right)+\frac{d+2}{2} .
\end{aligned}
$$

All terms of $\Delta_{A}\left(L_{1}\left(g_{1}\right) \otimes \ldots \otimes L_{n}\left(g_{n}\right)\right)$ are of the form (5.34) where $|b|$ is bounded by (5.46). Due to Lemma 9 the mass dimension of the functional (5.34) is bounded by

$$
\begin{gathered}
\sum_{i=1}^{n} \sum_{j_{i}=1}^{l_{i}}\left(\left|a_{i j_{i}}\right|+\frac{d-2}{2}\right)+\sum_{j=1}^{l}\left(\left|a_{j}\right|+\frac{d-2}{2}\right)+|b| \\
\leq \sum_{i=1}^{n} \operatorname{dim} L_{i}+\operatorname{dim} Q+\frac{d+2}{2}-d n .
\end{gathered}
$$

This implies the assertion (5.42).

\subsection{The MWI in the proper field formalism, and the Quantum Action Principle}

In the literature the Quantum Action Principle (QAP) is formulated in terms of time ordered products. Hence, to be able to compare our results with the QAP, we first rewrite Theorem 7 using $T$-products:

Lemma 11. Using the assumptions and notations of Theorem 7 the anomalous MWI (5.12) can equivalently be written in terms of the time-ordered product:

$$
T\left(e_{\otimes}^{i S / \hbar} \otimes\left(A+\delta_{A} S+\Delta_{A}\left(e_{\otimes}^{S}\right)\right)\right)=\int d y h(y) T\left(e_{\otimes}^{i S / \hbar} \otimes Q(y)\right) \frac{\delta S_{0}}{\delta \varphi(y)} .
$$

Proof. Using the formula of Bogoliubov (4.15) and the identity

$$
(F \star G) \cdot \frac{\delta S_{0}}{\delta \varphi}=F \star\left(G \cdot \frac{\delta S_{0}}{\delta \varphi}\right) \quad \forall F, G \in \mathcal{F}
$$

(cf. (5.23)), where $\cdot$ denotes the classical product, we obtain

$$
\begin{gathered}
\bar{T}\left(e_{\otimes}^{-i S / \hbar}\right) \star T\left(e_{\otimes}^{i S / \hbar} \otimes\left(A+\delta_{A} S+\Delta_{A}\left(e_{\otimes}^{S}\right)\right)\right)= \\
\bar{T}\left(e_{\otimes}^{-i S / \hbar}\right) \star\left(\int d y h(y) T\left(e_{\otimes}^{i S / \hbar} \otimes Q(y)\right) \cdot \frac{\delta S_{0}}{\delta \varphi(y)}\right) .
\end{gathered}
$$

Multiplication by $T\left(e_{\otimes}^{i S / \hbar}\right)$ from the left yields (5.48). 
As shown in (5.9) the MWI is satisfied by $T_{\text {tree }}$, i.e. it can be violated only by the contribution of the loop diagrams. To concentrate the study of the solvability of the MWI to that terms for which the MWI is a non-trivial condition, we reformulate the anomalous MWI (5.12) within the proper field formalism introduced in Sect. 4.

Corollary 12. The anomalous MWI in Theorem 7 can equivalently be expresses in terms of the vertex function $\Gamma_{T}$ (defined in Prop. 4):

$$
\int d x h(x) \Gamma_{T}\left(e_{\otimes}^{S} \otimes Q(x)\right) \frac{\delta\left(S_{0}+\Gamma_{T}\left(e_{\otimes}^{S}\right)\right)}{\delta \varphi(x)}=\Gamma_{T}\left(e_{\otimes}^{S} \otimes\left(A+\delta_{A} S+\Delta_{A}\left(e_{\otimes}^{S}\right)\right)\right) .
$$

Proof. Applying (4.19) on both sides of (5.48) we obtain

$$
\begin{aligned}
T_{\text {tree }}\left(e_{\otimes}^{i \Gamma_{T}\left(e_{\otimes}^{S}\right) / \hbar} \otimes\right. & \left.\Gamma_{T}\left(e_{\otimes}^{S} \otimes\left(A+\delta_{A} S+\Delta_{A}\left(e_{\otimes}^{S}\right)\right)\right)\right) \\
& =\int d y h(y) T_{\text {tree }}\left(e_{\otimes}^{i \Gamma_{T}\left(e_{\otimes}^{S}\right) / \hbar} \otimes \Gamma_{T}\left(e_{\otimes}^{S} \otimes Q(y)\right)\right) \frac{\delta S_{0}}{\delta \varphi(y)} .
\end{aligned}
$$

On the right-hand side we use the classical MWI in terms of $T$-products (5.9) and obtain

$$
\cdots=T_{\text {tree }}\left(e_{\otimes}^{i \Gamma_{T}\left(e_{\otimes}^{S}\right) / \hbar} \otimes \int d y h(y) \Gamma_{T}\left(e_{\otimes}^{S} \otimes Q(y)\right) \frac{\delta\left(S_{0}+\Gamma_{T}\left(e_{\otimes}^{S}\right)\right)}{\delta \varphi(y)}\right)
$$

which leads, by means of (4.13), to the assertion (5.51).

To compare this result with the literature, we introduce $\Gamma\left(S_{0}, S\right) \stackrel{\text { def }}{=} S_{0}+\Gamma_{T}\left(e_{\otimes}^{S}\right)$ and, motivated by (4.27) and (4.38), interpret $\Gamma\left(S_{0}, S\right)$ as the proper total action associated with the total classical action $S_{\text {tot }}=S_{0}+S$. In addition, we introduce 'insertions' (see [10]) in our algebraic formalism: let $P \in \mathcal{C}^{\infty}(\mathbb{M}, \mathcal{P})$ and $\rho \in \mathcal{D}(\mathbb{M})$ some testfunction (in the literature often called 'external field'). We define the 'insertion of $P(x)^{\prime}$, usually denoted ${ }^{13}$ by $P(x) \cdot \Gamma\left(S_{0}, S\right)$, as follows: ${ }^{14}$

$$
\left.P(x) \cdot \Gamma\left(S_{0}, S\right) \stackrel{\text { def }}{=} \frac{\delta}{\delta \rho(x)}\right|_{\rho \equiv 0} \Gamma\left(S_{0}, S+\int d x \rho(x) P(x)\right)=\Gamma_{T}\left(e_{\otimes}^{S} \otimes P(x)\right) .
$$

We denote by $S^{\prime} \stackrel{\text { def }}{=} S+\int d x \rho(x) Q(x), \rho \in \mathcal{D}(\mathbb{M})$ the modified classical interaction containing a coupling to the external field $\rho$, and write $\Delta_{A}\left(e_{\otimes}^{S}\right)=\int d x h(x) \tilde{\Delta}\left(e_{\otimes}^{S} ; Q(x)\right)$ with $\tilde{\Delta}\left(e_{\otimes}^{S} ; Q(x)\right) \in \mathcal{D}(\mathbb{M}, \mathcal{P})$ (Lemma $\left.8(i i)\right)$. Using this notation and the fact that the anomalous MWI (5.51) is valid for all $h \in \mathcal{D}(\mathbf{M})$, we rewrite (5.51) in terms of the proper total action:

$$
\left.\frac{\delta \Gamma\left(S_{0}, S^{\prime}\right)}{\delta \rho(x)} \frac{\delta \Gamma\left(S_{0}, S^{\prime}\right)}{\delta \varphi(x)}\right|_{\rho \equiv 0}=\Delta(x) \cdot \Gamma\left(S_{0}, S\right),
$$

\footnotetext{
${ }^{13}$ The dot does not mean the classical product here!

${ }^{14}$ Note, that in the setting of causal perturbation theory the introduction of external fields in order to express 'insertions' or nonlinear symmetry transformations is - in contrast to conventional perturbation theory - not necessary.
} 
where the local field $\Delta(x)$ is given by $\Delta(x) \stackrel{\text { def }}{=} Q(x) \frac{\delta\left(S_{0}+S\right)}{\delta \varphi(x)}+\tilde{\Delta}\left(e_{\otimes}^{S} ; Q(x)\right)$. This formulation of the anomalous MWI is formally equivalent to the general formulation of the QAP in [10]. Note that due to property $(v)$ in Theorem 7 and (4.27), the $\hbar$-expansion of the right-hand side of (5.53) starts with

$$
\Delta(x) \cdot \Gamma\left(S_{0}, S\right)=Q(x) \frac{\delta\left(S_{0}+S\right)}{\delta \varphi(x)}+\left.\mathcal{O}(\hbar) \equiv \frac{\delta\left(S_{0}+S^{\prime}\right)}{\delta \rho(x)} \frac{\delta\left(S_{0}+S^{\prime}\right)}{\delta \varphi(x)}\right|_{\rho=0}+\mathcal{O}(\hbar),
$$

which is completely analogous to the expansion of the right-hand side of the QAP in [10]. Moreover, for a renormalizable interaction $S$, the mass dimension of the local insertion $\Delta(x)$ is bounded by $\operatorname{dim} Q+\frac{d+2}{2}=\operatorname{dim} Q-\operatorname{dim} \varphi+d$ (Prop. 10), in agreement with [10].

\subsection{Removal of violations of the MWI}

\subsubsection{Fulfillment of the MWI to first order in $S$}

Before we are going to investigate the question whether the MWI can be fulfilled in a concrete model (i.e. for a given $A \in \mathcal{J}_{\text {loc }}$ and a given interaction $S \in \mathcal{F}_{\text {loc }}$ ), we will show that the MWI can always be fulfilled to first order in the interaction $S .{ }^{15}$ Apart from the interest in its own, this result will be needed in our proof of the Ward identities of the scalar $O(N)$-model (Sect. 5.4.4).

Proposition 13. Given $A=\int d y h(y) Q(y) \frac{\delta S_{0}}{\delta \varphi(y)}$ (with $\left.Q \in \mathcal{P}\right)$ and $S \in \mathcal{F}_{\text {loc }}$ there exists a retarded product $R_{1,1}$ which fulfills all renormalization conditions (specified in Sect. 3) and

$$
R_{1,1}(S, A)+\delta_{A} S=\int d y h(y) R_{1,1}(S, Q(y)) \frac{\delta S_{0}}{\delta \varphi(y)} .
$$

Proof. We start with $R$-products satisfying all renormalization conditions. For simplicity we work in the proper field formalism. ¿From the anomalous MWI (Cor. 12) we obtain to first order in $S$

$$
\Delta_{A}(S)=-\int d y h(y)\left(\Gamma_{T}\left(S \otimes Q(y) \frac{\delta S_{0}}{\delta \varphi(y)}\right)-\Gamma_{T}(S \otimes Q(y)) \frac{\delta S_{0}}{\delta \varphi(y)}\right) .
$$

By inserting the Wick expansion (3.7) of $\Gamma_{T}$ (which holds since $\Gamma_{T}$ fulfills Field Independence), we find that the terms containing no contraction of $\frac{\delta S_{0}}{\delta \varphi}$ cancel. It remains:

$$
\Delta_{A}(S)=-\int d x d y g(x) h(y) \sum_{\overline{\mathcal{L}} \subset \mathcal{L}, \bar{Q} \subset Q} \omega_{0}\left(\Gamma_{T}\left(\overline{\mathcal{L}}(x) \otimes \bar{Q}(y) \frac{\delta S_{0}}{\delta \varphi(y)}\right)\right) \underline{\mathcal{L}}(x) \underline{Q}(y)
$$

where we write $S=\int d x g(x) \mathcal{L}(x), g \in \mathcal{D}(\mathrm{M})$ and sum over all subpolynomials $\bar{P}=\frac{\partial^{l} P}{\partial\left(\partial^{a_{1}} \varphi\right) \cdots \partial\left(\partial^{a_{l}} \varphi\right)}$ of $P \in \mathcal{P}$ (for $\left.P=\mathcal{L}, Q\right)$, denoting by $\underline{P}=\frac{1}{l !} \prod_{j=1}^{l} \partial^{a_{j}} \varphi$ the corresponding 'counterpart' (see (3.7)). Due to locality of $\Delta_{A}(S)$ the distributions $\omega_{0}\left(\Gamma_{T}\left(\overline{\mathcal{L}}(x) \otimes \bar{Q}(y) \frac{\delta S_{0}}{\delta \varphi(y)}\right)\right)$ are supported on the diagonal $\{(x, y) \mid x=y\}$, cancellations

\footnotetext{
${ }^{15}$ E.g. the axial anomaly of QED is of second order in the interaction.
} 
of non-local terms on the right-hand side of (5.57) are impossible. We are searching a finite renormalization $\Gamma_{T} \rightarrow \hat{\Gamma}_{T}$ which removes the violating term $\Delta_{A}(S)$. Due to (4.33) a renormalization of $\Gamma_{T}(S \otimes F)$ must be of the form

$$
\hat{\Gamma}_{T}(S \otimes F)=\Gamma_{T}(S \otimes F)+D(S \otimes F), \quad \forall F \in \mathcal{F}_{\text {loc }},
$$

with $D$ satisfying the properties listed in the Main Theorem. To obtain a field independent $D$ we construct it from its vacuum expectation values by the causal Wick expansion (3.7). Obviously, setting

$$
\omega_{0}\left(D\left(\overline{\mathcal{L}}(x) \otimes \bar{Q}(y) \frac{\delta S_{0}}{\delta \varphi(y)}\right)\right) \stackrel{\text { def }}{=}-\omega_{0}\left(\Gamma_{T}\left(\overline{\mathcal{L}}(x) \otimes \bar{Q}(y) \frac{\delta S_{0}}{\delta \varphi(y)}\right)\right),
$$

the corresponding $\hat{\Gamma}_{T}$ satisfies the MWI to first order in $S$. Here we essentially use that the distribution on the right-hand side has local support. One verifies easily that $D$ (and hence the resulting $\hat{\Gamma}_{T}$ ) satisfies all renormalization conditions.

\subsubsection{Removal of possible anomalies by induction on the order in $\hbar$}

The formal equivalence of the anomalous MWI (written in the form of Cor. 12) and the QAP makes it possible to apply basic techniques of algebraic renormalization within the framework of causal perturbation theory. The main idea underlying algebraic renormalization is to start with an arbitrary renormalization prescription; with that the considered Ward identities are in general broken. Proceeding by induction on the power of $\hbar$, the question whether one can find a finite renormalization which removes the possible anomaly (i.e. the local terms violating the Ward identity) then amounts - by means of the QAP - to purely algebraic (often cohomological) problems.

We will apply this strategy to the MWI in the proper field formalism (which is given by (5.51) with $\Delta_{A}\left(e_{\otimes}^{S}\right)=0$ ): given $A \in \mathcal{J}_{\text {loc }}$ and $S \in \mathcal{F}_{\text {loc }}$, we are going to investigate whether possible anomalies of the corresponding MWI can be removed by finite renormalizations of $\Gamma_{T}$ (4.33). Proceeding by induction on the power of $\hbar$, we assume that for a given map $\Gamma_{T}^{(k)}$ (which satisfies the renormalization conditions Unitarity, $\mathcal{P}_{+}^{\uparrow}$-Covariance, Field Independence, Field Equation and Scaling Degree, see Lemma 6) the MWI is violated only by terms of order $\hbar^{k}$; that is in the anomalous MWI (Cor. 12) for $\Gamma_{T}^{(k)}$,

$$
\begin{gathered}
\Gamma_{T}^{(k)}\left(e_{\otimes}^{S} \otimes\left(A+\delta_{A} S\right)\right)+\Gamma_{T}^{(k)}\left(e_{\otimes}^{S} \otimes \Delta_{A}^{(k)}\left(e_{\otimes}^{S}\right)\right)= \\
\int d y h(y) \Gamma_{T}^{(k)}\left(e_{\otimes}^{S} \otimes Q(y)\right) \frac{\delta\left(S_{0}+\Gamma_{T}^{(k)}\left(e_{\otimes}^{S}\right)\right)}{\delta \varphi(y)},
\end{gathered}
$$

the violating term can be written as

$$
\Gamma_{T}^{(k)}\left(e_{\otimes}^{S} \otimes \Delta_{A}^{(k)}\left(e_{\otimes}^{S}\right)\right)=\Delta_{A}^{(k)}\left(e_{\otimes}^{S}\right)+\mathcal{O}\left(\hbar^{k+1}\right), \quad \Delta_{A}^{(k)}\left(e_{\otimes}^{S}\right)=\mathcal{O}\left(\hbar^{k}\right),
$$

where (4.27) is used. Note that, due to Theorem $7(v)$ and (4.27), any vertex function $\Gamma_{T}$ fulfills this assumption (5.60)-(5.61) for $k=1$ and, hence, can be used as $\Gamma_{T}^{(1)}$. 
To fulfill the MWI to $k^{\text {th }}$ order in $\hbar$ (and to maintain the other renormalization conditions), we have to find a renormalization map $D^{(k)}$ (with the properties $(i)-(v)$ and $(v i)(B)$ of the Main Theorem) such that

$$
\begin{aligned}
\Gamma_{T}^{(k+1)}\left(e_{\otimes}^{S} \otimes\left(A+\delta_{A} S\right)\right)+\mathcal{O}\left(\hbar^{k+1}\right)= \\
\quad \int d y h(y) \Gamma_{T}^{(k+1)}\left(e_{\otimes}^{S} \otimes Q(y)\right) \frac{\delta\left(S_{0}+\Gamma_{T}^{(k+1)}\left(e_{\otimes}^{S}\right)\right)}{\delta \varphi(y)},
\end{aligned}
$$

with $\Gamma_{T}^{(k+1)}$ given by

$$
\Gamma_{T}^{(k+1)}\left(e_{\otimes}^{F}\right)=\Gamma_{T}^{(k)}\left(e_{\otimes}^{D^{(k)}\left(e_{\otimes}^{F}\right)}\right) \quad \forall F \in \mathcal{F}_{\text {loc }} .
$$

To maintain the validity of the MWI to lower orders in $\hbar$ we choose $D^{(k)}$ to be of the form

$$
D^{(k)}\left(e_{\otimes}^{F}\right)=F+D_{>1}^{(k)}\left(e_{\otimes}^{F}\right), \quad D_{>1}^{(k)}\left(e_{\otimes}^{F}\right)=\mathcal{O}\left(\hbar^{k}\right), \quad \forall F \in \mathcal{F}_{\text {loc }}, F \sim \hbar^{0} .
$$

This implies

$$
e_{\otimes}^{D^{(k)}\left(e_{\otimes}^{F}\right)}=e_{\otimes}^{F}+e_{\otimes}^{F} \otimes_{\mathrm{sym}} D_{>1}^{(k)}\left(e_{\otimes}^{F}\right)+\mathcal{O}\left(\hbar^{k+1}\right)
$$

(where $\otimes_{\text {sym }}$ denotes the symmetrized tensor product) and

$$
\begin{gathered}
\Gamma_{T}^{(k+1)}\left(e_{\otimes}^{S}\right)=\Gamma_{T}^{(k)}\left(e_{\otimes}^{S}\right)+D_{>1}^{(k)}\left(e_{\otimes}^{S}\right)+\mathcal{O}\left(\hbar^{k+1}\right), \\
\Gamma_{T}^{(k+1)}\left(e_{\otimes}^{S} \otimes F\right)=\Gamma_{T}^{(k)}\left(e_{\otimes}^{S} \otimes F\right)+D_{>1}^{(k)}\left(e_{\otimes}^{S} \otimes F\right)+\mathcal{O}\left(\hbar^{k+1}\right) .
\end{gathered}
$$

We insert the latter two equations into our requirement (5.62) and use the inductive assumption (5.60)-(5.61). It results

$$
\begin{aligned}
\Delta_{A}^{(k)}\left(e_{\otimes}^{S}\right)= & D_{>1}^{(k)}\left(e_{\otimes}^{S} \otimes\left(A+\delta_{A} S\right)\right)-\delta_{A} D_{>1}^{(k)}\left(e_{\otimes}^{S}\right) \\
& -\int d y h(y) D_{>1}^{(k)}\left(e_{\otimes}^{S} \otimes Q(y)\right) \frac{\delta\left(S_{0}+S\right)}{\delta \varphi(y)}+\mathcal{O}\left(\hbar^{k+1}\right)
\end{aligned}
$$

The violating term $\Delta_{A}^{(k)}\left(e_{\otimes}^{S}\right)$ is inductively given by $\Gamma_{T}^{(k)}$. If we succeed to find a corresponding map $D_{>1}^{(k)}$ fulfilling (5.68) and the properties of a renormalization map, then the pertinent finite renormalization removes the 'anomaly' $\Delta_{A}^{(k)}\left(e_{\otimes}^{S}\right)$. However, since $D_{>1}^{(k)}$ appears in (5.68) several times with different arguments it seems almost impossible to discuss the existence of solutions in general.

\subsubsection{Assumption: localized off-shell version of Noether's Theorem}

In various important applications of the MWI the search for solutions $D_{>1}^{(k)}$ of (5.68) is simplified due to the validity of the following assumption. In the given model the total action ${ }^{16}$

$S_{0}+S(g)\left(\right.$ with $\left.S(g)=\sum_{n \geq 1} \kappa^{n} S_{n}(g), S_{n}(g)=\int d x(g(x))^{n} \mathcal{L}_{n}(x), g \in \mathcal{D}(\mathbb{M}), \mathcal{L}_{n} \in \mathcal{P}\right)$

\footnotetext{
${ }^{16} \kappa$ denotes the coupling constant.
} 
is invariant with respect to the symmetry transformation

$\delta_{A}=\int d y h(y) Q(y) \frac{\delta}{\delta \varphi(y)}=\sum_{n \geq 0} \kappa^{n} \delta_{A_{n}}$ (corresponding to $A=\sum_{n \geq 0} \kappa^{n} A_{n}, A_{n} \in \mathcal{J}_{\text {loc }}$ )

in the following way: there exist

- a current $j^{\mu}(g)=\sum_{n \geq 0} \kappa^{n} j_{n}^{\mu}(g), j_{n}^{\mu}(g)(x)=(g(x))^{n} j_{n}^{\mu}(x)$ (with $\left.j_{n}^{\mu} \in \mathcal{P}\right)$ and

- a 'Q-vertex' ${ }^{17} \mathcal{L}^{(1) \mu}(g)=\sum_{n \geq 1} \kappa^{n} \mathcal{L}_{n}^{(1) \mu}(g), \quad \mathcal{L}_{n}^{(1) \mu}(g)(x)=(g(x))^{n-1} \mathcal{L}_{n}^{(1) \mu}(x)$ $\left(\right.$ with $\left.\mathcal{L}_{n}^{(1) \mu} \in \mathcal{P}\right)$

such that

$$
\delta_{A}\left(S_{0}+S(g)\right) \equiv A+\delta_{A} S(g)=\int d x h(x)\left(\partial_{\mu} j^{\mu}(g)(x)-\mathcal{L}^{(1) \mu}(g)(x) \partial_{\mu} g(x)\right)
$$

in the sense of formal power series in $\kappa$. To zeroth order in $\kappa$ the assumption (5.69) reads

$$
\delta_{A_{0}} S_{0} \equiv A_{0}=\int d x h(x) \partial_{\mu} j_{0}^{\mu}(x),
$$

where $j_{0}$ is the symmetry current of the underlying free theory. This simplifying assumption can be interpreted as the validity of an off-shell version of Noether's Theorem for the case that the interaction and the symmetry transformation are localized. It is satisfied e.g. for the scalar $O(N)$-model treated in Sect. 5.4.4 and for the BRSTsymmetry of (massless) Yang-Mills theories, massive spin-1 fields and massless spin-2 fields (gravity). For the interacting scalar $O(N)$-model (see Sect. 5.4.4) the simplifications $A=A_{0}, \delta_{A} S=0, \mathcal{L}^{(1)}=0$ and $j=j_{0}$ appear. However, for the BRST-symmetry $\delta_{A}$ and $j$ are generically non-trivial deformations of $\delta_{A_{0}}$ and $j_{0}$, and $\delta_{A} S$ and $\mathcal{L}^{(1)}$ do not vanish.

Example: BRST-symmetry. We are going to verify that the above mentioned models satisfy the assumption (5.69). Our argumentation is based on conservation of the classical BRST-current. For constant couplings (i.e. $g(x)=1 \forall x$ ) there is a conserved Noether current $j=\sum_{n \geq 0} \kappa^{n} j_{n}$, due to the BRST-invariance of the total action. We use these $j_{n}$ 's to construct the BRST-current of the corresponding model with localized coupling $\kappa g(x)(g \in \mathcal{D}(\mathbb{M}))$ : we set $j(g)(x):=\sum_{n>0}(\kappa g(x))^{n} j_{n}(x)$. The violation of the conservation of $j(g)$ is expressed in terms of the $Q$-vertex $[13,2,1]$ : in Sects. 3 and 4 of [27] it is shown that for the considered models there exists a $Q$-vertex $\mathcal{L}^{(1)}(g)$ such that

$$
R_{\mathrm{cl}}\left(e_{\otimes}^{S(g)}, \int d x h(x)\left(\partial j(g)(x)-\mathcal{L}^{(1) \mu}(g)(x) \partial_{\mu} g(x)\right)\right) \in \mathcal{J} .
$$

Proceeding by induction on the order in $\kappa$ and using the MWI (as it is worked out in formulas (190)-(191) and (152)-(157) of [1]) one finds that (5.71) is equivalent to the sequence of relations

$$
A_{0}:=\int d x h(x) \partial j_{0}(x) \in \mathcal{J}_{\text {loc }}
$$

\footnotetext{
${ }^{17}$ The name 'Q-vertex' is due to 'perturbative gauge invariance' [31], which is related to BRSTsymmetry.
} 
and

$$
A_{n}:=-\sum_{l=0}^{n-1} \delta_{A_{l}} S_{n-l}(g)+\int d x h(x)\left(\partial j_{n}(g)(x)-\mathcal{L}_{n}^{(1)}(g)(x) \partial g(x)\right) \in \mathcal{J}_{\mathrm{loc}}
$$

for $n \geq 1$. This yields our assumption: with (5.72) the condition (5.70) holds true and (5.73) implies $A_{n}=\delta_{A_{n}} S_{0}$ and with that (5.73) gives (5.69) to $n$th order in $\kappa$. $\delta_{A}=\sum_{n \geq 0} \kappa^{n} \delta_{A_{n}}$ is a localized version of the usual BRST-transformation.

We point out that the nilpotency of the BRST-transformation is not directly used. Every model fulfilling the local current conservation (5.71) (with $S(g), j(g)$ and $\mathcal{L}^{(1)}(g)$ of the above form) satisfies our assumption (5.69) if the symmetry transformation $\delta_{A}$ is defined by (5.72)-(5.73).

Assuming now the validity of (5.69), we are going to derive a simplified version of (5.68). For shortness and coincidence with the notations of the preceding Sects. we write $S, j$ and $\mathcal{L}^{(1)}$ for $S(g), j(g)$ and $\mathcal{L}^{(1)}(g)$, respectively. For the time being, we additionally assume that the testfunction $h$ satisfies $h(x)=1$ for all $x \in \operatorname{supp}(\delta S / \delta \varphi)$. With that $\Delta_{A}^{(k)}\left(e_{\otimes}^{S}\right)$ is independent of the choice of $h$ within this class and $h$ can be replaced by the number 1 (see Lemma $8(i i))$. $h$ does not appear also on the right-hand side of (5.68): namely, due to our assumption and the locality of the map $D_{>1}^{(k)}$ (see Theorem 3(i)) we obtain

$$
D_{>1}^{(k)}\left(e_{\otimes}^{S} \otimes\left(A+\delta_{A} S\right)\right)=\int d y(\partial_{\mu}^{y} \underbrace{D_{>1}^{(k)}\left(e_{\otimes}^{S} \otimes j^{\mu}(y)\right)}_{=0 \text { for } y \notin \operatorname{supp}(\delta S / \delta \varphi)}-D_{>1}^{(k)}\left(e_{\otimes}^{S} \otimes \mathcal{L}^{(1) \mu}(y)\right) \partial_{\mu} g(y))
$$

and, using again the locality of $D_{>1}^{(k)},(5.68)$ simplifies to the condition

$$
\begin{gathered}
\Delta^{(k)}\left(e_{\otimes}^{S}\right)=-\int d y\left(D_{>1}^{(k)}\left(e_{\otimes}^{S} \otimes Q(y)\right) \frac{\delta\left(S_{0}+S\right)}{\delta \varphi(y)}+D_{>1}^{(k)}\left(e_{\otimes}^{S} \otimes \mathcal{L}^{(1)}(y)\right) \partial g(y)\right) \\
-\delta D_{>1}^{(k)}\left(e_{\otimes}^{S}\right)+\mathcal{O}\left(\hbar^{k+1}\right)
\end{gathered}
$$

where $\left.\Delta^{(k)}\left(e_{\otimes}^{S}\right) \stackrel{\text { def }}{=} \Delta_{A}^{(k)}\left(e_{\otimes}^{S}\right)\right|_{h \equiv 1}$ and $\delta$ is the non-localized version of $\delta_{A}$ :

$$
\delta \stackrel{\text { def }}{=} \int d x Q(x) \frac{\delta}{\delta \varphi(x)} .
$$

It is much easier to find a solution $D_{>1}^{(k)}$ for (5.75) than for (5.68), since in (5.75) the localization of the derivation $\delta_{A}$ is removed (i.e. $h$ is replaced by 1 ) and since the $D_{>1}^{(k)}\left(e_{\otimes}^{S} \otimes \partial j\right)$-term vanishes.

However, we want to solve the MWI for general $h \in \mathcal{D}(\mathbb{M}){ }^{18}$ To investigate whether (5.75) is also sufficient for the more involved condition (5.68) for arbitrary $h$, let a solution $D_{>1}^{(k)}$ of $(5.75)$ be given. We point out that the map $D_{>1}^{(k)}$ is not

\footnotetext{
${ }^{18}$ For example this is used in the derivation of our version of the QAP (5.53) from the anomalous MWI.
} 
completely determined by that, only the combination of $D_{>1}^{(k)}\left(e_{\otimes}^{S}\right), D_{>1}^{(k)}\left(e_{\otimes}^{S} \otimes Q\right)$ and $D_{>1}^{(k)}\left(e_{\otimes}^{S} \otimes \mathcal{L}^{(1)}\right)$ appearing on the right-hand side of (5.75) is fixed. We claim that, given such a $D_{>1}^{(k)}$, there exists a linear map $K^{\mu}: \mathbb{T} \mathcal{F}_{\text {loc }} \rightarrow \mathcal{D}(\mathbb{M}, \mathcal{P})$ with the property

$$
\begin{gathered}
\Delta_{A}^{(k)}\left(e_{\otimes}^{S}\right)=\int d y h(y)\left(\partial_{\mu} K^{\mu}\left(e_{\otimes}^{S}\right)(y)-D_{>1}^{(k)}\left(e_{\otimes}^{S} \otimes Q(y)\right) \frac{\delta\left(S_{0}+S\right)}{\delta \varphi(y)}\right. \\
\left.-D_{>1}^{(k)}\left(e_{\otimes}^{S} \otimes \mathcal{L}^{(1)}(y) \partial g(y)\right)\right)-\delta_{A} D_{>1}^{(k)}\left(e_{\otimes}^{S}\right)+\mathcal{O}\left(\hbar^{k+1}\right)
\end{gathered}
$$

for all $h \in \mathcal{D}(\mathbb{M})$ and with $K^{\mu}(1)=0$. The latter condition is compatible with (5.77), because to zeroth order in $\kappa$ the condition (5.77) reduces to $\int h \partial K(1)=\mathcal{O}\left(\hbar^{k+1}\right)$, due to $D_{>1}^{(k)}(1 \otimes F)=D_{>1}^{(k)}(F)=0(\forall F$, see $(5.64))$.

To show the existence of $K^{\mu}$, we first prove the following Lemma, which describes the difference between $\delta_{A}$ and $\delta$ with respect to their action on local functionals:

Lemma 14. Let be given $F \in \mathcal{F}_{\text {loc }}, l \in \mathcal{D}(\mathbb{M}, \mathcal{P})$ and a localized derivation $\delta_{h}=$ $\int d x h(x) Q(x) \frac{\delta}{\delta \varphi(x)}, h \in \mathcal{D}(\mathbb{M}), Q \in \mathcal{P}$, such that the corresponding non-localized derivation $\delta=\int d x Q(x) \frac{\delta}{\delta \varphi(x)}$ satisfies

$$
\delta F=\int d x l(x)
$$

Then there exists a $k^{\mu} \in \mathcal{D}(\mathbb{M}, \mathcal{P})$ such that the following localized version of (5.78) holds true:

$$
\delta_{h} F=\int d x h(x)\left(l(x)+\partial_{\mu} k^{\mu}(x)\right) .
$$

Proof. Let $f \in \mathcal{D}(\mathbb{M}, \mathcal{P})$ with $F=\int d y f(y)$. Carrying out the functional derivative in (5.78) we conclude

$$
l(x)=\sum_{a \in \mathbb{N}_{0}^{d}} \partial^{a} Q(x) \frac{\partial f}{\partial\left(\partial^{a} \varphi\right)}(x)+\partial_{\mu} k_{1}^{\mu}(x)
$$

for some $k_{1}^{\mu} \in \mathcal{D}(\mathbb{M}, \mathcal{P})$. On the other hand we obtain

$$
\begin{aligned}
\delta_{h} F & =\int d x \sum_{a \in \mathbb{N}_{0}^{d}} \partial^{a}(h(x) Q(x)) \frac{\partial f}{\partial\left(\partial^{a} \varphi\right)}(x) \\
& =\int d x h(x) \sum_{a \in \mathbb{N}_{0}^{d}} \partial^{a} Q(x) \frac{\partial f}{\partial\left(\partial^{a} \varphi\right)}(x)+\int d x h(x) \partial_{\mu} k_{2}^{\mu}(x)
\end{aligned}
$$

for some other $k_{2}^{\mu} \in \mathcal{D}(\mathbb{M}, \mathcal{P})$. Hence, setting $k^{\mu}=-k_{1}^{\mu}+k_{2}^{\mu}$ we obtain the assertion (5.79).

To prove (5.77) we use that $\Delta_{A}^{(k)}\left(e_{\otimes}^{S}\right)$ can be written as

$\Delta_{A}^{(k)}\left(e_{\otimes}^{S}\right)=\int d x h(x) \tilde{\Delta}^{(k)}\left(e_{\otimes}^{S} ; Q(x)\right) \quad$ with $\quad \tilde{\Delta}^{(k)}\left(e_{\otimes}^{S} ; Q(x)\right) \in \mathcal{D}(\mathbb{M}, \mathcal{P})$, 
due to Lemma 8(ii). Hence, (5.75) can be written in the form $\delta F=\int l+\mathcal{O}\left(\hbar^{k+1}\right)$ (5.78) with $F=D_{>1}^{(k)}\left(e_{\otimes}^{S}\right)$ and

$-l(x)=D_{>1}^{(k)}\left(e_{\otimes}^{S} \otimes Q(x)\right) \frac{\delta\left(S_{0}+S\right)}{\delta \varphi(x)}+D_{>1}^{(k)}\left(e_{\otimes}^{S} \otimes \mathcal{L}^{(1)}(x)\right) \partial g(x)+\tilde{\Delta}^{(k)}\left(e_{\otimes}^{S} ; Q(x)\right) \in \mathcal{D}(\mathbb{M}, \mathcal{P})$.

With that Lemma 14 yields our assertion (5.77).

We conclude that a solution of (5.68) can be obtained from a solution of (5.75) by setting

$$
D_{>1}^{(k)}\left(e_{\otimes}^{S} \otimes j^{\mu}(y)\right) \stackrel{\text { def }}{=} K^{\mu}\left(e_{\otimes}^{S}\right)(y)
$$

provided this does not lead to any contradictions with the partial fixing of $D$ in terms of $D_{>1}^{(k)}\left(e_{\otimes}^{S}\right), D_{>1}^{(k)}\left(e_{\otimes}^{S} \otimes Q\right)$ and $D_{>1}^{(k)}\left(e_{\otimes}^{S} \otimes \mathcal{L}^{(1)}\right)$. Due to the causal Wick expansion (3.7) and the Field equation $D_{>1}^{(k)}\left(e_{\otimes}^{S} \otimes \partial^{a} \varphi\right)=0$ (see Theorem 3,(iii)), this is the case whenever the intersection of the subpolynomials of $j$ with the subpolynomials of $Q, \mathcal{L}^{(1)}$ or $\mathcal{L}_{n}(\forall n \in \mathbb{N})$ contains only numbers and terms which are linear in the field $\varphi$ itself or partial derivatives thereof. ${ }^{19}$ Note that (5.84) satisfies the condition $D_{>1}^{(k)}(j)=0$.

Remark: If $Q$ is linear in $\varphi$, the maintenance of the Field Equation requires $D_{>1}^{(k)}\left(e_{\otimes}^{S} \otimes\right.$ $Q)=0$ (Theorem 3). With that the right-handed side of (5.75) vanishes to first order in $\kappa$ up to terms of order $\hbar^{k+1}$. That is the condition (5.75) can only be satisfied if $\Delta^{(k)}\left(S_{1}\right)=\mathcal{O}\left(\hbar^{k+1}\right)$. Hence, following the proof of Proposition 13, we first perform a finite renormalization which maintains the considered renormalization conditions and removes the term $\sim \hbar^{k}$ of $\Delta_{A}^{(k)}\left(S_{1}\right)$. This can be done such that $\Delta_{A}^{(k)}\left(e_{\circledast}^{S}\right)=\mathcal{O}\left(\hbar^{k}\right)$ is preserved. Namely, since $\Delta_{A}^{(k)}\left(S_{1}\right)=\mathcal{O}\left(\hbar^{k}\right)$ the vacuum expectation values on the right-hand side of (5.57) are of order $\hbar^{k}$ and, hence, the pertinent renormalization map $D(5.59)$ can be chosen of the form (5.64).

\subsubsection{Proof of the Ward identities in the $O(N)$ scalar field model}

In the case of compact internal symmetry groups, covariance can be obtained by integration over the group. To illustrate the developed formalism we proceed alternatively.

We will prove that an off-shell generalization of the Ward identities expressing current conservation in a scalar $O(N)$-model can be fulfilled to all orders of perturbation theory. Our strategy is based partially on techniques of algebraic renormalization described in detail in [10].

We consider a multiplet of $N$ scalar fields $\varphi_{i}(x), i=1, \ldots, N$, transforming under the defining representation of $O(N)$ - the group of orthogonal $N \times N$ matrices -20

$$
\varphi_{i} \longrightarrow A_{i j} \varphi_{j} \quad, \quad A \in O(N) \cdot{ }^{21}
$$

\footnotetext{
${ }^{19}$ In QED this condition is not satisfied; one has to discuss the individual cases to see that the definition (5.84) does not lead to contradictions (see e.g. [13]).

${ }^{20}$ By setting $\varphi=\frac{1}{\sqrt{2}}\left(\varphi_{1}+i \varphi_{2}\right)$ and $\bar{\varphi}=\frac{1}{\sqrt{2}}\left(\varphi_{1}-i \varphi_{2}\right)$ the $O(2)$ model can be seen to be equivalent to the well known $U(1)$ model of a complex scalar field $\varphi$.

${ }^{21}$ repeated indices are summed over;
} 
Let $\left\{X^{a} \mid a=1, \ldots, \frac{1}{2} N(N-1)\right\}$ be a basis of the Lie algebra $\mathfrak{o}(N)$ of $O(N)$, and $f^{a b c}$ the corresponding structure constants,

$$
\left[X^{a}, X^{b}\right]=f^{a b c} X^{c} .
$$

The dynamics of our model is given by the free action

$$
S_{0}=\frac{1}{2} \int d x\left(\partial^{\mu} \varphi_{i}(x) \partial_{\mu} \varphi_{i}(x)-m^{2} \varphi_{i}(x) \varphi_{i}(x)\right)
$$

and the localized, $O(N)$-invariant interaction

$$
S=\int d x g(x)\left(\varphi_{i}(x) \varphi_{i}(x)\right)^{2} \quad \text { with } \quad g \in \mathcal{D}(\mathbb{M}) .
$$

Since the free action $S_{0}$ is invariant under the transformation (5.85), there exist $\frac{1}{2} N(N-1)$ conserved Noether currents $j_{\mu}^{a}=X_{i j}^{a} \varphi_{j} \partial_{\mu} \varphi_{i}$, i.e. the local functionals $A^{a} \stackrel{\text { def }}{=} \int d x h(x) \partial^{\mu} j_{\mu}^{a}(x)$ (with arbitrary $h \in \mathcal{D}(\mathbb{M})$ ) are elements of the ideal $\mathcal{J}$ generated by the free field equations:

$$
A^{a}=\delta_{A^{a}} S_{0} \in \mathcal{J}, \quad \text { with } \quad \delta_{A^{a}}=\int d x h(x) X_{i j}^{a} \varphi_{j}(x) \frac{\delta}{\delta \varphi_{i}(x)} .
$$

Essential simplifications of this model are the validity of (5.69) in simplified form and additionally that $Q(5.10)$ is linear in $\varphi$.

The conservation of the interacting currents $\left(j_{\mu}^{a}\right)_{S}=R\left(e_{\otimes}^{S}, j_{\mu}^{a}\right)$ follows from the MWI for the given $A^{a}$ and interaction $S$ :

$$
\int d x h(x) \partial^{\mu} R\left(e_{\otimes}^{S}, j_{\mu}^{a}(x)\right) \equiv R\left(e_{\otimes}^{S}, A^{a}\right)=\int d x h(x) R\left(e_{\otimes}^{S}, X_{i j}^{a} \varphi_{j}(x)\right) \frac{\delta S_{0}}{\delta \varphi_{i}(x)} .
$$

Regarding the question whether (5.90) can be fulfilled to all orders, we start with an $R$-product satisfying the renormalization conditions Unitarity, $\mathcal{P}_{+}^{\uparrow}$-Covariance, Field Independence, Field Equation and Scaling Degree. Then, the Ward identities (5.90) may be violated; however, Theorem 7 guarantees the existence of local maps $\Delta_{A^{a}}$ : $\mathbb{T} \mathcal{F}_{\text {loc }} \rightarrow \mathcal{F}_{\text {loc }}$ such that

$$
R\left(e_{\otimes}^{S}, A^{a}+\Delta_{A^{a}}\left(e_{\otimes}^{S}\right)\right)=\int d x h(x) R\left(e_{\otimes}^{S}, X_{i j}^{a} \varphi_{j}(x)\right) \frac{\delta S_{0}}{\delta \varphi_{i}(x)} .
$$

To find a finite renormalization of the $R$-product which removes $R\left(e_{\otimes}^{S}, \Delta_{A^{a}}\left(e_{\otimes}^{S}\right)\right)$, we follow the technique described in the preceding sections: we assume the existence of a $\Gamma_{T}^{(k)}$ such that (5.90) is fulfilled up to terms of order $\hbar^{k}$, i.e.

$$
\Gamma_{T}^{(k)}\left(e_{\otimes}^{S} \otimes A^{a}\right)+\Delta_{A^{a}}^{(k)}\left(e_{\otimes}^{S}\right)+\mathcal{O}\left(\hbar^{k+1}\right)=\int d y h(y) X_{i j}^{a} \varphi_{j}(y) \frac{\delta\left(S_{0}+\Gamma_{T}^{(k)}\left(e_{\otimes}^{S}\right)\right)}{\delta \varphi_{i}(y)}
$$

where $\Delta_{A^{a}}^{(k)}\left(e_{\otimes}^{S}\right)=\mathcal{O}\left(\hbar^{k}\right)$. (The Field Equation for $\Gamma_{T}^{(k)}$ (Lemma 6) is taken into account.) First we perform a finite renormalization which maintains $\Delta_{A^{a}}^{(k)}\left(e_{\otimes}^{S}\right)=\mathcal{O}\left(\hbar^{k}\right)$ 
and the mentioned renormalization conditions and which removes the terms $\sim \hbar^{k}$ of $\Delta_{A^{a}}^{(k)}(S)$. Due to the requirement $D_{>1}^{(k)}\left(e_{\otimes}^{S} \otimes \varphi\right)=0$ (Theorem 3,(iii)), the condition (5.75) simplifies to

$$
\Delta_{a}^{(k)}\left(e_{\otimes}^{S}\right)=-\delta_{a} D_{>1}^{(k)}\left(e_{\otimes}^{S}\right)+\mathcal{O}\left(\hbar^{k+1}\right),
$$

where we set $\left.\Delta_{a}^{(k)}\left(e_{\otimes}^{S}\right) \stackrel{\text { def }}{=} \Delta_{A^{a}}^{(k)}\left(e_{\otimes}^{S}\right)\right|_{h \equiv 1}$ and $\delta_{a} \stackrel{\text { def }}{=} \int d x X_{i j}^{a} \varphi_{j}(x) \frac{\delta}{\delta \varphi_{i}(x)}$. To fulfill (5.90) up to terms of order $\hbar^{k+1}$, we have to solve (5.93) and to extend the definition of this $D_{>1}^{(k)}$ in such a way that condition (5.68) (for general $h$ ) holds true. The latter can be done by means of (5.84), because the intersection of the non-trivial subpolynomials of $j_{\mu}^{a}$ with the subpolynomials of $Q$ or $\left(\varphi_{i} \varphi_{i}\right)^{2}$ is a subset of $\mathbb{C} \varphi$.

It remains to show the solvability of (5.93). For this purpose we temporarily restrict the functionals $(2.1)$ in $(5.92)$ to the space $\mathcal{D}(\mathbb{M})$ of compactly supported test functions on Minkowski space. This permits us to perform the $\operatorname{limit}^{22} h \rightarrow 1$ in (5.92), ending up with the equation

$$
\delta_{a}\left(S_{0}+\Gamma_{T}^{(k)}\left(e_{\otimes}^{S}\right)\right)=\Delta_{a}^{(k)}\left(e_{\otimes}^{S}\right)+\mathcal{O}\left(\hbar^{k+1}\right) .
$$

Furthermore, using (5.86) we obtain the identity

$$
\left[\delta_{a}, \delta_{b}\right]=f_{a b c} \delta_{c}
$$

which we insert into $\left[\delta_{a}, \delta_{b}\right]\left(S_{0}+\Gamma_{T}^{(k)}\left(e_{\otimes}^{S}\right)\right)$. This yields the consistency condition

$$
\delta_{a} \Delta_{b}^{(k)}\left(e_{\otimes}^{S}\right)-\delta_{b} \Delta_{a}^{(k)}\left(e_{\otimes}^{S}\right)=f_{a b c} \Delta_{c}^{(k)}\left(e_{\otimes}^{S}\right)+\mathcal{O}\left(\hbar^{k+1}\right)
$$

Due to the compact support of $S$, and the locality of $\Delta_{a}^{(k)}$, each term in (5.96) has compact support as well. Therefore, this equation holds true on the entire configuration space $\mathcal{C}(\mathbb{M}, \mathbb{R})$, i.e. the restriction of the functionals to $\mathcal{D}(\mathbb{M})$ can be omitted.

The consistency condition (5.96) is the cocycle condition in the Lie algebra cohomology corresponding to the Lie algebra generated by the derivations $\left\{\delta_{a}\right\}$ acting on $\mathcal{F}_{\text {loc }}$. Trivial solutions are the coboundaries,

$$
\Delta_{a}^{(k)}\left(e_{\otimes}^{S}\right)=-\delta_{a} \hat{\Delta}^{(k)}\left(e_{\otimes}^{S}\right)+\mathcal{O}\left(\hbar^{k+1}\right)
$$

for some linear, symmetric and local map $\hat{\Delta}^{(k)}: \mathbb{T} \mathcal{F}_{\text {loc }} \rightarrow \mathcal{F}_{\text {loc. }}$ If $\Delta_{a}^{(k)}\left(e_{\otimes}^{S}\right)$ is a coboundary, the condition (5.93) can be solved by setting

$$
D_{>1}^{(k)}(1) \stackrel{\text { def }}{=} 0, \quad D_{>1}^{(k)}(S) \stackrel{\text { def }}{=} 0 \quad \text { and } \quad D_{>1}^{(k)}\left(S^{\otimes j}\right) \stackrel{\text { def }}{=} \hat{\Delta}^{(k)}\left(S^{\otimes j}\right) \quad \forall j \geq 2,
$$

due to $\Delta_{a}^{(k)}(S)=\mathcal{O}\left(\hbar^{k+1}\right)$.

Hence, we only have to show that the present cohomology is trivial. In the literature (see e.g. [10] and references therein) it is shown that every Lie algebra cohomology corresponding to some semi-simple Lie group and some finite dimensional representation is trivial. This result applies to our problem. Namely, $O(N)$ is semi-simple for $N>2$ and, since the mass dimension of $\Delta_{a}^{(k)}\left(e_{\otimes}^{S}\right)$ is bounded (5.43), the anomaly terms indeed span a finite dimensional representation of $\mathfrak{o}(N)$. It does not matter that our functionals are local. Note that $D_{>1}^{(k)}\left(e_{\otimes}^{S}\right)$ is not uniquely defined by this procedure.

\footnotetext{
${ }^{22}$ This limit is done as follows: let $h \in \mathcal{D}(\mathbb{M})$ such that there is a neighbourhood $U$ of $0(\in \mathbb{M})$ with $\left.h\right|_{U}=1$. Then we replace $h(x)$ by $h_{\epsilon}(x) \equiv h(\epsilon x)(\epsilon>0)$ and perform the limit $\epsilon \rightarrow 0$.
} 


\section{Conclusions and Outlook}

In algebraic renormalization the QAP is used to remove possible anomalies of Ward identities by induction on $\hbar$. We have worked out an analogous procedure for the MWI in the different framework of causal perturbation theory. The main difference is that we work solely with compactly supported interactions $S$ and localized symmetry transformations $\delta_{A} \cdot{ }^{23}$ Our main result gives a crucial insight into the structure of possible anomalies of the MWI, in particular with respect to the deformation parameter $\hbar$, and allows the transfer of techniques from algebraic renormalization into causal perturbation theory.

This yields a general method to fulfill the MWI for a given model. A first nontrivial application is worked out (Sect. 5.4.4). The developed method seems to be applicable to many models (as suggested by [2], [11] and [10]). Together with the powerful tool of BRST cohomology it should make possible a proof of that cases of the MWI which are needed for the construction of the net of local observables of Yang-Mills type QFTs. (This would complete the construction given in [2].) A main advantage of this approach to quantum Yang-Mills theories is that there seems to be no serious obstacle for the generalization to curved spacetimes where the techniques developed for scalar fields in [12] and [32] can be used. For recent and far-reaching progress in the construction of renormalized quantum Yang-Mills fields in curved spacetime see [33]; this paper uses a generalization of the off-shell Master BRST Identity (i.e. the MWI for the symmetry transformation $\delta_{A_{0}}$ with $A_{0}$ given by $(5.72)$, see $[1,2]$ for the on-shell version) to models with antifields.

\section{A Proper vertices for $R$-products}

\section{A.1 Definition and basic properties}

Before we introduce proper vertices in terms of $R$-products, we shortly consider the diagrammatics of retarded products $R\left(A_{1}\left(x_{1}\right), \ldots ; A_{n}\left(x_{n}\right)\right)$.

For the unrenormalized expressions (i.e. for $x_{i} \neq x_{j}, \forall i \neq j$ ) the diagrammatic interpretation is unique. One can show that there are two kinds of inner lines which symbolize $\Delta^{\text {ret }}$ and $H_{m}^{\mu}$, respectively, and are oriented. For tree diagrams only $\Delta^{\text {ret }}$ appears and all inner lines are pointing to the distinguished vertex $A_{n}\left(x_{n}\right)$. Solely connected diagrams contribute to $R$; and $R_{\mathrm{cl}}$ is precisely the contribution of all tree diagrams. Both statements follow from the inductive construction of the $\left(R_{n, 1}\right)_{n \in \mathbb{N}}$ [16]. The decisive step is the GLZ Relation: $\left\{F_{G}, H_{G}\right\}=\ldots$. In the quantum case there is at least one contraction between $F_{G}$ and $H_{G}$, and in classical FT there is precisely one contraction in $\left\{F_{G}, H_{G}\right\}_{\mathrm{cl}}$.

For the renormalized retarded product we use these results as definition of the connected and tree part: $R^{c} \equiv R$ and $R_{\text {tree }} \equiv R_{\mathrm{cl}}$.

\footnotetext{
${ }^{23}$ Algebraic renormalization applies to global and local symmetries; examples for local symmetries which have been dealt are current algebras of $\sigma$-models and current algebras of gauge theories in which one keeps external fields (e.g. antifields).
} 
Analogously to (4.12)-(4.14) the property $R_{(\mathrm{cl})}(1, F)=F$ implies the following conclusions

$$
\begin{gathered}
R_{(\mathrm{cl})}\left(e_{\otimes}^{\sum_{n=1}^{\infty} F_{n} \lambda^{n}}, \sum_{n=0}^{\infty} G_{n} \lambda^{n}\right)=0 \Longrightarrow G_{n}=0 \quad \forall n \\
R_{(\mathrm{cl})}\left(e_{\otimes}^{\sum_{n=1}^{\infty} F_{n} \lambda^{n}}, \sum_{n=0}^{\infty} \frac{\delta F_{n}}{\delta \varphi} \lambda^{n}\right)=R_{(\mathrm{cl})}\left(e_{\otimes}^{\sum_{n=1}^{\infty} G_{n} \lambda^{n}}, \sum_{n=0}^{\infty} \frac{\delta G_{n}}{\delta \varphi} \lambda^{n}\right) \\
\wedge \omega_{0}\left(F_{n}\right)=\omega_{0}\left(G_{n}\right) \forall n \Longrightarrow F_{n}=G_{n} \quad \forall n,
\end{gathered}
$$

which hold for the $R$-products of classical FT $\left(R_{\mathrm{cl}}\right)$ and of QFT $(R)$. In classical FT the statement (A.1) can be proved also non-perturbatively: $0=R_{\mathrm{cl}}\left(e_{\otimes}^{F(\lambda)}, G(\lambda)\right)=$ $G(\lambda) \circ r_{S_{0}+F(\lambda), S_{0}}$ implies $G(\lambda)=G(\lambda) \circ r_{S_{0}+F(\lambda), S_{0}} \circ r_{S_{0}, S_{0}+F(\lambda)}=0$.

The concept of proper vertices has a clear physical interpretation when applied to $R$-products, since $R_{\text {tree }}=R_{\mathrm{cl}}$. As explained in Sect. 2 the entries of $R_{\mathrm{cl}}$ may be non-local. We want to rewrite an interacting QFT-field $R\left(e_{\otimes}^{S}, F\right)$ as a classical field $R_{\mathrm{cl}}\left(e_{\otimes}^{\Gamma_{R}\left(e_{\otimes}^{S}\right)}, \Gamma_{\mathrm{ret}}\left(e_{\otimes}^{S}, F\right)\right)$ where the 'proper interaction' $\Gamma_{R}\left(e_{\otimes}^{S}\right)$ and the 'proper retarded field' $\Gamma_{\text {ret }}\left(e_{\otimes}^{S}, F\right)$ are non-local and agree to lowest order in $\hbar$ with the original local functionals $S$ and $F$ respectively. This is indeed possible:

Proposition 15. (a) There exist

- a totally symmetric and linear map

$$
\Gamma_{R}: \mathbb{T} \mathcal{F}_{\text {loc }} \longrightarrow \mathcal{F}
$$

- and a linear map

$$
\Gamma_{\text {ret }}: \mathbb{T} \mathcal{F}_{\text {loc }} \otimes \mathcal{F}_{\text {loc }} \longrightarrow \mathcal{F}
$$

which is totally symmetric in the former entries (i.e. $\quad \Gamma_{\text {ret }}\left(\otimes_{j=1}^{n} F_{\pi j}, F\right)=$ $\left.\Gamma_{\text {ret }}\left(\otimes_{j=1}^{n} F_{j}, F\right)\right)$,

which are uniquely determined by the conditions

$$
\begin{gathered}
R\left(e_{\otimes}^{S}, F\right)=R_{\mathrm{cl}}\left(e_{\otimes}^{\Gamma_{R}\left(e_{\otimes}^{S}\right)}, \Gamma_{\mathrm{ret}}\left(e_{\otimes}^{S}, F\right)\right), \\
\Gamma_{\mathrm{ret}}\left(e_{\otimes}^{S}, \varphi(h)\right)=\varphi(h), \quad h \in \mathcal{D}(\mathbb{M}), \\
\Gamma_{R}(\mathbf{1})=0, \quad \omega_{0}\left(\Gamma_{R}\left(e_{\otimes}^{S}\right)\right)=0 .
\end{gathered}
$$

(b) $\Gamma_{R}$ and $\Gamma_{\text {ret }}$ are related by

$$
\frac{\delta \Gamma_{R}\left(e_{\otimes}^{S}\right)}{\delta \varphi(x)}=\Gamma_{\text {ret }}\left(e_{\otimes}^{S}, \frac{\delta S}{\delta \varphi(x)}\right),
$$

that is, with (A.7), $\Gamma_{R}$ is uniquely determined by $\Gamma_{\text {ret }}$. 
Compared with the defining condition (4.19) for $\Gamma_{T}$, there is more flexibility in (A.5) since it contains two kinds of 'vertex functions', $\Gamma_{R}$ and $\Gamma_{\text {ret }}$. To define the latter uniquely, we additionally require (A.6) and (A.7).

Proof. (b) First we show that the defining conditions for $\Gamma_{R}, \Gamma_{\text {ret }}$ given in part (a) imply the statement in part (b). The off-shell field equation

$$
R_{(\mathrm{cl})}\left(e_{\otimes}^{S}, \frac{\delta S_{0}}{\delta \varphi(x)}\right)=\frac{\delta S_{0}}{\delta \varphi(x)}-R_{(\mathrm{cl})}\left(e_{\otimes}^{S}, \frac{\delta S}{\delta \varphi(x)}\right),
$$

holds for $R(\mathrm{QFT})$ and $R_{\mathrm{cl}}$ (classical FT), in the latter case even for non-local entries. With that and using the conditions (A.5) and (A.6) and finally (A.1) we obtain the assertion (A.8).

(a) By expanding (A.5) in powers of $S$ and using (A.8) and (A.7) we find an inductive construction of $\Gamma_{R}$ and $\Gamma_{\text {ret }}$ in terms of $R$ and $R_{\mathrm{cl}}$ :

$$
\begin{gathered}
\Gamma_{\text {ret }}(1, F)=R(F) \equiv F \\
\frac{\delta \Gamma_{R}(S)}{\delta \varphi(x)}=\Gamma_{\text {ret }}\left(1, \frac{\delta S}{\delta \varphi(x)}\right)=\frac{\delta S}{\delta \varphi(x)} \Longrightarrow \Gamma_{R}(S)=S, \\
\Gamma_{\text {ret }}(S, F)=R(S, F)-R_{\mathrm{cl}}(S, F) \\
\frac{\delta \Gamma_{R}\left(S^{\otimes 2}\right)}{\delta \varphi(x)}=2 \Gamma_{\text {ret }}\left(S, \frac{\delta S}{\delta \varphi(x)}\right)=2 R\left(S, \frac{\delta S}{\delta \varphi(x)}\right)-2 R_{\mathrm{cl}}\left(S, \frac{\delta S}{\delta \varphi(x)}\right), \\
\Gamma_{\text {ret }}\left(S^{\otimes 2}, F\right)=R\left(S^{\otimes 2}, F\right)-R_{\mathrm{cl}}\left(S^{\otimes 2}, F\right)-R_{\mathrm{cl}}\left(\Gamma_{R}\left(S^{\otimes 2}\right), F\right)-2 R_{\mathrm{cl}}\left(S, \Gamma_{\text {ret }}(S, F)\right) .
\end{gathered}
$$

We explicitly see that $\Gamma_{\text {ret }}$ is not totally symmetric, it is retarded with respect to the last entry.

Now let $\Gamma_{R}\left(e_{\otimes}^{S}\right)$ and $\Gamma_{\text {ret }}\left(e_{\otimes}^{S}, F\right)$ be constructed up to order $n$ and $(n-1)$ respectively in $S$. Then, the condition (A.5) determines $\Gamma_{\text {ret }}\left(S^{\otimes n}, F\right)$ uniquely:

$$
\begin{gathered}
\Gamma_{\mathrm{ret}}\left(S^{\otimes n}, F\right)=R\left(S^{\otimes n}, F\right)-\sum_{k=1}^{n}\left(\begin{array}{l}
n \\
k
\end{array}\right) R_{\mathrm{cl}}\left(\left.\frac{d^{k}}{d \lambda^{k}}\right|_{\lambda=0} e_{\otimes}^{\Gamma_{R}\left(e_{\otimes}^{\lambda S}\right)}, \Gamma_{\mathrm{ret}}\left(S^{\otimes n-k}, F\right)\right) \\
=R\left(S^{\otimes n}, F\right)-\sum_{k=1}^{n}\left(\begin{array}{l}
n \\
k
\end{array}\right) \sum_{\substack { j=1 \\
\begin{subarray}{c}{l_{1}, \ldots, l_{j}=1 \\
l_{1}+\cdots+l_{j}=k{ j = 1 \\
\begin{subarray} { c } { l _ { 1 } , \ldots , l _ { j } = 1 \\
l _ { 1 } + \cdots + l _ { j } = k } }\end{subarray}}^{k} \frac{1}{j ! l_{1} ! \cdots l_{j} !} R_{\mathrm{cl}}\left(\bigotimes_{i=1}^{j} \Gamma_{R}\left(S^{\otimes l_{i}}\right), \Gamma_{\mathrm{ret}}\left(S^{\otimes n-k}, F\right)\right) .
\end{gathered}
$$

¿From that and with (A.8) and (A.7) we uniquely get $\Gamma_{R}\left(S^{\otimes n+1}\right)$.

Remark: The roles of the conditions (A.6) and (A.8) can be exchanged. In the list (A.5)-(A.7) of defining conditions, (A.6) can be replaced by (A.8). Then, (A.6) can be derived from (A.5), (A.7) and (A.8) analogously to (4.30) and (4.31): proceeding inductively we use (A.11), the integrated field equation for $R$ and $R_{\mathrm{cl}}$ and (A.8).

Following the construction (A.10)-(A.11) we inductively prove the following properties of $\Gamma_{R}, \Gamma_{\text {ret }}$ : 
- $\hbar$-dependence:

$$
\left.\begin{array}{rl}
\Gamma_{\text {ret }}\left(e_{\otimes}^{S}, F\right) & =F+\mathcal{O}(\hbar) \\
\Gamma_{R}\left(e_{\otimes}^{S}\right) & =S+\mathcal{O}(\hbar)
\end{array}\right\} \quad \text { if } \quad F, S \sim \hbar^{0}
$$

- $\mathcal{P}_{+}^{\uparrow}$-Covariance, Unitarity $\left(\Gamma_{R}\left(e_{\otimes}^{S}\right)^{*}=\Gamma_{R}\left(e_{\otimes}^{S^{*}}\right)\right.$ and similarly for $\left.\Gamma_{\text {ret }}\right)$, Field Independence, Smoothness in $m \geq 0, \mu$-Covariance and Almost homogeneous Scaling of $\Gamma_{R} \equiv \Gamma_{R}^{(m, \mu)}$ and $\Gamma_{\text {ret }} \equiv \Gamma_{\text {ret }}^{(m, \mu)}$ (or, alternatively, Scaling Degree).

In the proof of (A.12) we use $R=R_{\mathrm{cl}}+\mathcal{O}(\hbar), R_{\mathrm{cl}} \sim \hbar^{0}$. The other properties rely on the validity of the corresponding axioms for $R$ and $R_{\mathrm{cl}}$, analogously to Lemma 6 .

We point out that $\Gamma_{R}\left(e_{\otimes}^{S} \otimes \varphi(h)\right)$ differs in general from $\varphi(h)$, in contrast to the Field equation for $\Gamma_{T}$ and (A.6). Namely, inserting (A.5)-(A.6) into the GLZ-relation for $\left[R\left(e_{\otimes}^{S}, \varphi(h)\right), R\left(e_{\otimes}^{S}, \varphi(g)\right)\right]$ we obtain

$$
\begin{gathered}
\frac{i}{\hbar}\left[R_{\mathrm{cl}}\left(e_{\otimes}^{\Gamma_{R}\left(e_{\otimes}^{S}\right)}, \varphi(h)\right), R_{\mathrm{cl}}\left(e_{\otimes}^{\Gamma_{R}\left(e_{\otimes}^{S}\right)}, \varphi(g)\right)\right]= \\
R_{\mathrm{cl}}\left(e_{\otimes}^{\Gamma_{R}\left(e_{\otimes}^{S}\right)} \otimes \Gamma_{R}\left(e_{\otimes}^{S} \otimes \varphi(g)\right), \varphi(h)\right)-R_{\mathrm{cl}}\left(e_{\otimes}^{\Gamma_{R}\left(e_{\otimes}^{S}\right)} \otimes \Gamma_{R}\left(e_{\otimes}^{S} \otimes \varphi(h)\right), \varphi(g)\right) .
\end{gathered}
$$

Due to the GLZ Relation for $R_{\mathrm{cl}}$, the left-hand side is equal to

$$
\begin{gathered}
R_{\mathrm{cl}}\left(e_{\otimes}^{\Gamma_{R}\left(e_{\otimes}^{S}\right)} \otimes \varphi(g), \varphi(h)\right)-R_{\mathrm{cl}}\left(e_{\otimes}^{\Gamma_{R}\left(e_{\otimes}^{S}\right)} \otimes \varphi(h), \varphi(g)\right) \\
+\frac{i}{\hbar}\left[R_{\mathrm{cl}}\left(e_{\otimes}^{\Gamma_{R}\left(e_{\otimes}^{S}\right)}, \varphi(h)\right), R_{\mathrm{cl}}\left(e_{\otimes}^{\Gamma_{R}\left(e_{\otimes}^{S}\right)}, \varphi(g)\right)\right]_{\star(\geq 2)},
\end{gathered}
$$

where $\left.\frac{i}{\hbar}[\cdot, \cdot]_{\star} \geq 2\right) \equiv \frac{i}{\hbar}[\cdot, \cdot]_{\star}-\{\cdot, \cdot\}_{\mathrm{cl}}$. The assertion follows from the non-vanishing of the $[\cdot, \cdot]_{\star}(\geq 2)$-term.

Finally we study a finite renormalization $R \rightarrow \hat{R}$. To express the corresponding renormalizations of $\Gamma_{R}$ and $\Gamma_{\text {ret }}$ in terms of the corresponding map $D$ of the Main Theorem we insert the defining relation (A.5) into both sides of (3.23). In the resulting equation

$$
R_{\mathrm{cl}}\left(e_{\otimes}^{\hat{\Gamma}_{R}\left(e_{\otimes}^{S}\right)}, \hat{\Gamma}_{\text {ret }}\left(e_{\otimes}^{S}, F\right)\right)=R_{\mathrm{cl}}\left(e_{\otimes}^{\Gamma_{R}\left(e_{\otimes}^{D\left(e_{\otimes}^{S}\right)}\right)}, \Gamma_{\mathrm{ret}}\left(e_{\otimes}^{D\left(e_{\otimes}^{S}\right)}, D\left(e_{\otimes}^{S} \otimes F\right)\right)\right)
$$

we choose $F=\frac{\delta S}{\delta \varphi}$. With that we may replace $\hat{\Gamma}_{\text {ret }}\left(e_{\otimes}^{S}, F\right)$ by $\frac{\delta \hat{\Gamma}_{R}\left(e_{\otimes}^{S}\right)}{\delta \varphi}$ and $\Gamma_{\text {ret }}\left(e_{\otimes}^{D\left(e_{\otimes}^{S}\right)}, D\left(e_{\otimes}^{S} \otimes F\right)\right)$ by $\frac{\delta \Gamma_{R}\left(e_{\otimes}^{D\left(e_{\otimes}^{S}\right)}\right)}{\delta \varphi}$. By means of (A.2) we conclude

$$
\hat{\Gamma}_{R}\left(e_{\otimes}^{S}\right)=\Gamma_{R}\left(e_{\otimes}^{D\left(e_{\otimes}^{S}\right)}\right) .
$$

We insert this into (A.15) and apply (A.1). This yields

$$
\hat{\Gamma}_{\text {ret }}\left(e_{\otimes}^{S}, F\right)=\Gamma_{\text {ret }}\left(e_{\otimes}^{D\left(e_{\otimes}^{S}\right)}, D\left(e_{\otimes}^{S} \otimes F\right)\right) .
$$




\section{A.2 Comparison of the vertex functions in terms of $T$ - and $R$-products}

The vertex functions $\Gamma_{T}$ and $\Gamma_{R}$ defined in terms of $T$ - and $R$-products, respectively, are both totally symmetric, nevertheless they do not agree. This follows from the different forms of the unitarity property or, alternatively, from the non-validity of $\Gamma_{T}\left(e_{\otimes}^{S} \otimes \varphi(h)\right)=\varphi(h)$ for $\Gamma_{R}$. We are going to compare $\Gamma_{T}$ with $\Gamma_{R}$ to lowest orders in $S$.

By using the definitions of $\Gamma_{R}, \Gamma_{\text {ret }}$ (A.5) and $\Gamma_{T}$ (4.19), as well as (4.15)-(4.16) we obtain

$$
\begin{gathered}
R_{\mathrm{cl}}\left(e_{\otimes}^{\Gamma_{R}\left(e_{\otimes}^{S}\right)}, \Gamma_{\text {ret }}\left(e_{\otimes}^{S}, F\right)\right)= \\
\sum_{n=0}^{\infty}\left(1-T_{\text {tree }}\left(e_{\otimes}^{i \Gamma_{T}\left(e_{\otimes}^{S}\right) / \hbar}\right)\right)^{\star n} \star T_{\text {tree }}\left(e_{\otimes}^{i \Gamma_{T}\left(e_{\otimes}^{S}\right) / \hbar} \otimes \Gamma_{T}\left(e_{\otimes}^{S} \otimes F\right)\right) .
\end{gathered}
$$

If we interpret $\Gamma_{R}, \Gamma_{\text {ret }}$ and $\Gamma_{T}$ as one vertex, then the l.h.s. contains solely tree diagrams, but on the r.h.s. there appear also loop diagrams! This indicates that the relation of $\Gamma_{R}$ to $\Gamma_{T}$ is rather involved. To zeroth and first order in $S$ we obtain

$$
\begin{gathered}
\Gamma_{\text {ret }}(1, F)=\Gamma_{T}(1 \otimes F)=F \\
\Gamma_{\text {ret }}(S, F)+R_{\mathrm{cl}}(S, F)=i / \hbar T_{\text {tree }}(S \otimes F)+\Gamma_{T}(S \otimes F)-i / \hbar S \star F
\end{gathered}
$$

The terms $\sim \hbar^{-1}$ and $\sim \hbar^{0}$ of (4.15) read

$$
R_{\mathrm{cl}}(S, F)=i / \hbar T_{\text {tree }}(S \otimes F)-i / \hbar S \star^{(\leq 1)} F,
$$

where

$$
\begin{aligned}
S \star^{(\leq 1)} F= & \sum_{n \leq 1} \frac{\hbar^{n}}{n !} \int d x_{1} \ldots d x_{n} d y_{1} \ldots d y_{n} \frac{\delta^{n} S}{\delta \varphi\left(x_{1}\right) \cdots \delta \varphi\left(x_{n}\right)} \\
& \cdot \prod_{i=1}^{n} H_{m}^{\mu}\left(x_{i}-y_{i}\right) \frac{\delta^{n} F}{\delta \varphi\left(y_{1}\right) \cdots \delta \varphi\left(y_{n}\right)} .
\end{aligned}
$$

In the same way we define $S \star^{(\geq 2)} F$ (i.e. $S \star^{2}=S \star^{(\leq 1)} F+S \star^{(\geq 2)} F$ ) and e.g. $S \star^{(2)} F$. With that (A.20) reads

$$
\begin{gathered}
\Gamma_{T}(S \otimes F)=\Gamma_{\text {ret }}(S, F)+i / \hbar S \star^{(\geq 2)} F \\
=\frac{1}{2}\left(\Gamma_{\text {ret }}(S, F)+\Gamma_{\text {ret }}(F, S)+i / \hbar\left(S \star^{(\geq 2)} F+F \star^{(\geq 2)} S\right)\right) .
\end{gathered}
$$

Using additionally the Field Independence of $\Gamma_{T}$ and (A.10d) we find

$$
\frac{\delta}{\delta \varphi}\left(\Gamma_{T}(S \otimes F)-\Gamma_{R}(S \otimes F)\right)=\frac{i}{\hbar}\left(S \star^{(\geq 2)} \frac{\delta F}{\delta \varphi}+F \star^{(\geq 2)} \frac{\delta S}{\delta \varphi}\right) .
$$


Selecting the terms of second order in $S$ from (A.18) we find

$$
\begin{gathered}
1 / 2 \Gamma_{\text {ret }}\left(S^{\otimes 2}, F\right)+R_{\mathrm{cl}}\left(S, \Gamma_{\text {ret }}(S, F)\right)+1 / 2 R_{\mathrm{cl}}\left(S^{\otimes 2}, F\right)+1 / 2 R_{\mathrm{cl}}\left(\Gamma_{R}\left(S^{\otimes 2}\right), F\right)= \\
1 / 2 \Gamma_{T}\left(S^{\otimes 2} \otimes F\right)+i / \hbar T_{\text {tree }}\left(S \otimes \Gamma_{T}(S \otimes F)\right)+i / 2 \hbar T_{\text {tree }}\left(\Gamma_{T}\left(S^{\otimes 2}\right) \otimes F\right) \\
-1 / 2 \hbar^{2} T_{\text {tree }}\left(S^{\otimes 2} \otimes F\right)-i / \hbar S \star \Gamma_{T}(S \otimes F)+1 / \hbar^{2} S \star T_{\text {tree }}(S \otimes F) \\
-i / 2 \hbar \Gamma_{T}\left(S^{\otimes 2}\right) \star F+1 / 2 \hbar^{2} T_{\text {tree }}\left(S^{\otimes 2}\right) \star F-1 / \hbar^{2} S \star S \star F .
\end{gathered}
$$

To simplify this formula and to eliminate all vertex functions $\Gamma_{\text {ret }}, \Gamma_{R}, \Gamma_{T}$ with two arguments, we use (A.23), (A.10d), the Field Independence, as well as

$$
R_{\mathrm{cl}}(G, H)=\int d x d y \frac{\delta H}{\delta \varphi(x)} \Delta^{\mathrm{ret}}(x-y) \frac{\delta G}{\delta \varphi(y)}
$$

and the corresponding expression for $T_{\text {tree }}^{c}(G, H)$, in addition (A.21) and the corresponding identity

$$
\begin{gathered}
1 / 2 R_{\mathrm{cl}}\left(S^{\otimes 2}, F\right)=\frac{1}{\hbar^{2}}\left(-1 / 2 T_{\text {tree }}\left(S^{\otimes 2} \otimes F\right)\right. \\
\left.+(S \star T(S \otimes F))_{\text {tree }}+1 / 2\left(T\left(S^{\otimes 2}\right) \star F\right)_{\text {tree }}+(S \star S \star F)_{\text {tree }}\right),
\end{gathered}
$$

and also

$$
T_{\text {tree }}(S \otimes F)-i \hbar \Gamma_{T}(S \otimes F)=T(S \otimes F)
$$

(which follows from Corollary 5 and (4.11)). It results

$$
\begin{gathered}
1 / 2\left(\Gamma_{\text {ret }}\left(S^{\otimes 2}, F\right)-\Gamma_{T}\left(S^{\otimes 2} \otimes F\right)\right)= \\
\frac{i}{\hbar} \int d x d y \frac{\delta F}{\delta \varphi(x)} \Delta^{\mathrm{ret}}(x-y)\left(S \star^{(\geq 2)} \frac{\delta S}{\delta \varphi(y)}\right)+\frac{i}{\hbar} R_{\mathrm{cl}}\left(S, S \star^{(\geq 2)} F\right) \\
-\frac{1}{\hbar^{2}} \sum_{a+b=3}\left(S \star^{(\geq a)} S \star^{(\geq b)} F\right)^{c}+\frac{1}{\hbar^{2}}\left(\left(S \star^{(\geq 2)} T(S \otimes F)\right)^{c}-\left(S \star^{(2)}(S \cdot F)\right)_{\text {tree }}\right) \\
+\frac{1}{2 \hbar^{2}}\left(\left(T\left(S^{\otimes 2}\right) \star^{(\geq 2)} F\right)^{c}-\left((S \cdot S) \star^{(2)} F\right)_{\text {tree }}\right)
\end{gathered}
$$

In comparison with (A.25) a main simplification is that on the right-hand side solely connected diagrams contribute and the cancellation of all tree diagrams is obvious (i.e. the right-hand side is manifestly of order $\hbar$ ).

We have not succeeded to generalize the results (A.23), (A.24) and (A.29) to a general formula relating $\Gamma_{T}$ to $\Gamma_{\text {ret }}$ or $\Gamma_{R}$.

Acknowledgment: This paper is to a large extent based on the diploma thesis of one of us (F.B.) [34], which was supervised by Klaus Fredenhagen. We profitted from discussions with him in many respects: he gave us important ideas, technical help and also suggestions for the presentation of the material. We are grateful also to Raymond Stora for valuable and detailed comments on the manuscript, which we used to improve some formulations. 


\section{References}

[1] Dütsch, M. and Fredenhagen, K. "The Master Ward identity and generalized Schwinger-Dyson equation in classical field theory". Commun. Math. Phys. 243 (2003) 275-314.

[2] Dütsch, M. and Boas, F. M. "The Master Ward identity". Rev. Math. Phys. 14 (2002) 977 .

[3] Lowenstein, J. H. "Differential vertex operations in Lagrangian field theory". Commun. Math. Phys. 24 (1971) 1-21.

[4] Lam, Y.-M. P. "Perturbation Lagrangian theory for scalar fields: Ward- Takahasi identity and current algebra". Phys. Rev. D6 (1972) 2145-2161.

[5] Breitenlohner, P. and Maison, D. "Dimensional renormalization and the action principle". Commun. Math. Phys. 52 (1977) 11-38.

[6] Becchi, C., Rouet, A. and Stora, R. "Renormalization of the abelian Higgs-Kibble Model". Commun. Math. Phys. 42 (1975) 127-162.

[7] Becchi, C., Rouet, A. and Stora, R. "Renormalization of gauge theories". Annals Phys. 98 (1976) 287-321.

[8] Zinn-Justin, J. "Renormalization of gauge theories and master equation". Mod. Phys. Lett. A14 (1999) 1227-1236.

[9] Henneaux, M. and Teitelboim, C. "Quantization of gauge systems" (1992) 1-520. Princeton University Press, Princeton, New Jersey.

[10] Piguet, O. and Sorella, S. P. Algebraic renormalization: Perturbative renormalization, symmetries and anomalies, vol. M28 of Lect. Notes Phys.. 1995.

[11] Barnich, G., Brandt, F. and Henneaux, M. "Local BRST cohomology in gauge theories". Phys. Rept. 338 (2000) 439-569.

[12] Brunetti, R. and Fredenhagen, K. "Microlocal analysis and interacting quantum field theories: Renormalization on physical backgrounds". Commun. Math. Phys. 208 (2000) 623-661.

[13] Dütsch, M. and Fredenhagen, K. "A local (perturbative) construction of observables in gauge theories: The example of QED". Commun. Math. Phys. 203 (1999) $71-105$.

[14] Dütsch, M. and Fredenhagen, K. "Perturbative algebraic field theory, and deformation quantization". Fields Institute Communications 30 (2001) 151-160.

[15] Dütsch, M. and Fredenhagen, K. "Algebraic quantum field theory, perturbation theory, and the loop expansion". Commun. Math. Phys. 219 (2001) 5-30. 
[16] Dütsch, M. and Fredenhagen, K. "Causal perturbation theory in terms of retarded products, and a proof of the Action Ward identity". Rev. Math. Phys. 16 (2004) 1291-1348.

[17] Bogoliubov, N. and Shirkov, D. Introduction to the Theory of Quantized Fields. 1959. New York.

[18] Epstein, H. and Glaser, V. "The role of locality in perturbation theory". Annales Poincare Phys. Theor. A19 (1973) 211.

[19] Brunetti, R., Dütsch, M. and Fredenhagen, K. "Retarded products versus timeordered products: a geometrical interpretation" work in preparation.

[20] Dütsch, M. and Fredenhagen, K. "Action Ward identity and the StückelbergPetermann renormalization group". In Boutet de Monvel, A., Iagolnitzer, D. and Moschella, U. (Eds.) "Rigorous Quantum Field Theory", pp. 113-123. Birkhäuser Verlag, 2006. hep-th/0501228.

[21] Peierls, R. "The commutation laws of relativistic field theory". Proc. Roy. Soc.(London) A 214 (1952) 143.

[22] Marolf, D. M. "The Generalized Peierls bracket”. Ann. Phys. 236 (1994) 392-412.

[23] Steinmann, O. Perturbative Expansion in axiomatic field theory, vol. 11 of Lect. Notes Phys.. 1971.

[24] Steinmann, O. Perturbative quantum electrodynamics and axiomatic field theory. Berlin, Germany: Springer (2000) 355 p.

[25] Itzykson, C. and Zuber, J.-B. Quantum Field Theory. New York: Mc Graw-Hill (1980).

[26] Dütsch, M., Hurth, T., Krahe, F. and Scharf, G. "Causal Construction of YangMills theories. I." N. Cimento A 106 (1993) 1029.

[27] Dütsch, M. "Proof of perturbative gauge invariance for tree diagrams to all orders". Ann. Phys. (Leipzig) 14 (2005) 438-461.

[28] Jona-Lasinio, G. "Relativistic Field Theories with Symmetry-Breaking Solutions". Nuovo Cimento 34 (1964) 1790-1795.

[29] Gell-Mann, M. and Low, F. "Bound States in Quantum Field Theory". Physical Review 84 (1951) 350-354.

[30] Brennecke, F. and Dütsch, M. "The Quantum Action Principle in the framework of Causal Perturbation Theory". hep-th/0801.1408.

[31] Dütsch, M., Hurth, T., Krahe, F. and Scharf, G. "Causal Construction of YangMills theories. II." N. Cimento A 107 (1994) 375.

[32] Hollands, S. and Wald, R. M. "Conservation of the stress tensor in interacting quantum field theory in curved spacetimes". Rev. Math. Phys. 17 (2005) 227-312. 
[33] Hollands, S. "Renormalized Quantum Yang-Mills Fields in Curved Spacetime" (2007). gr-qc/0705.3340.

[34] Brennecke, F. "Investigations to the anomaly problem of the Master Ward Identity". diploma thesis (in german) (2005). http://www.desy.de/unith/lqp/psfiles/dipl-brennecke.ps.gz. 